University of Louisville

ThinkIR: The University of Louisville's Institutional Repository

Electronic Theses and Dissertations

$5-2014$

\title{
SA-4-1BBL as a platform to develop adjuvant systems for prophylactic and therapeutic vaccines.
}

Gunes Dinc 1984-

University of Louisville

Follow this and additional works at: https://ir.library.louisville.edu/etd

Part of the Immunology and Infectious Disease Commons, and the Microbiology Commons

\section{Recommended Citation}

Dinc, Gunes 1984-, "SA-4-1BBL as a platform to develop adjuvant systems for prophylactic and therapeutic vaccines." (2014). Electronic Theses and Dissertations. Paper 350.

https://doi.org/10.18297/etd/350

This Doctoral Dissertation is brought to you for free and open access by ThinkIR: The University of Louisville's Institutional Repository. It has been accepted for inclusion in Electronic Theses and Dissertations by an authorized administrator of ThinkIR: The University of Louisville's Institutional Repository. This title appears here courtesy of the author, who has retained all other copyrights. For more information, please contact thinkir@louisville.edu. 


\title{
SA-4-1BBL AS A PLATFORM TO DEVELOP ADJUVANT SYSTEMS FOR PROPHYLACTIC AND THERAPEUTIC VACCINES
}

\author{
By \\ Gunes Dinc \\ B.S., Uludag University, 2007 \\ M.S., University of Louisville, 2011

\begin{abstract}
A Dissertation
Submitted to the Faculty of the in Partial Fulfillment of the Requirements for the Degree of

Doctor of Philosophy

Department of Microbiology and Immunology

University of Louisville

Louisville, KY
\end{abstract} \\ School of Medicine of the University of Louisville
}

May 2014 
Copyright 2014 by Gunes Dinc

All rights reserved 



\title{
SA-4-1BBL AS A PLATFORM TO DEVELOP ADJUVANT SYSTEMS FOR PROPHYLACTIC AND THERAPEUTIC VACCINES
}

\author{
By \\ Gunes Dinc \\ B.S., Uludag University, 2007 \\ M.S., University of Louisville, 2011 \\ A Dissertation Approved on
}

March 13, 2014

by the following Dissertation Committee:

\begin{tabular}{c}
\hline Dr. Haval Shirwan \\
\hline Dr. Esma Shirwan Yolcu \\
\hline Dr. Haribabu Bodduluri \\
\hline
\end{tabular}

Dr. Jun Yan

Dr. Matthew B. Lawrenz 


\section{DEDICATION}

This dissertation is dedicated to my parents

Mrs. Ozcan Dinc and Mr. Kerim Dinc

and my fiancé Mr. Onur Akbulut

who have given me invaluable support, understanding, and eternal love from overseas,

and

waited for me with patience. 


\section{ACKNOWLEDGEMENTS}

First of all, I would like to thank my advisor Dr. Haval Shirwan, for his guidance, patience, and encouragement. I am very grateful that he taught me how to become a true scientist, do troubleshooting, and find my path in this journey. I also would like to thank my Co-mentor Dr. Esma S. Yolcu who has been very supportive and helpful, not only in the laboratory, but also outside. I am very lucky to learn from her both in professional and personal life.

I am very thankful to my committee members Dr. Haribabu Bodduluri, Dr. Jun Yan, and Dr. Matthew Lawrenz for their support, guidance and time. A special thanks to Department of Microbiology and Immunology faculty, staff, and students, as well as ICT for providing me excellent support to pursue my $\mathrm{PhD}$. I also would like to thank Turkish Ministry of National Education for providing the scholarship to pursue my Doctoral degree.

I am very grateful to all of my lab members who have been very supportive and helpful during this education. I am thankful to Orlando, Hong, HB, and Kyle for their support, help, and most importantly friendship.

Finally, I cannot end this section without mentioning my parents, and my fiancé's

tremendous support. Whatever I say that cannot translate my feeling. I am very lucky and thankful to have them. 


\begin{abstract}
SA-4-1BBL AS A PLATFORM TO DEVELOP ADJUVANT SYSTEMS FOR PROPHYLACTIC AND THERAPEUTIC VACCINES
\end{abstract}

Gunes Dinc

March 13, 2014

Vaccines against infectious diseases are one of the most critical accomplishments in modern medicine. Despite significant progress in vaccinology, there is still a dire need for developing vaccines against various acute and chronic infections and cancer. In general, vaccines are categorized as prophylactic, given to healthy individuals to prevent disease, and therapeutic, administered to people who already have disease. As such, the nature, quality, and quantity of immune responses required for the efficacy of these two types vaccines are different. Prophylactic vaccines against infectious diseases primarily rely on the generation of neutralizing high titers of antibody for their efficacy. These vaccines are generally effective because they target a host with an unaltered and competent immune system. In marked contrast, the efficacy of therapeutic vaccines has been a major challenge since they are administered into a host with a compromised immune system. Therapeutic vaccines need not only to generate effective adaptive cellular, particularly $\mathrm{CD}^{+} \mathrm{T}$ cell, immune responses to chronic infection and cancer, but they also need to overcome various immune evasion mechanisms employed by infection 
and progressing tumor. For both types of vaccines, the generation of a long-lasting adaptive immunity is the key.

Historically, prophylactic vaccines against infections were made from liveattenuated or inactivated forms of the microbes, but there were concerns about stability, side effects and safety of such vaccines. Advancements in molecular biology and DNA technologies led to the development of recombinant subunit vaccine with well-defined antigens. In particular, vaccines based on recombinant proteins present an attractive approach because of their ease of production, storage, distribution, and safety profiles. However, recombinant protein based subunit vaccines are poorly immunogenic and require adjuvants for efficacy. Most of the adjuvants that have been approved for clinical use, and those under development primarily target innate arm of the immune system for the generation of subsequent adaptive immunity. Key to the initiation of adaptive immune responses is the interactions between an APC and T cells and acquisition of 3 distinct singles by T cells. Signal 1 is delivered by the interaction of TCR on T cells with an $\mathrm{MHC}$ /peptide complex on APC. This signal is then qualified by costimulatory receptor ligand interaction on the APC and T cells, providing signal 2. Signal 3 is provided by various cytokines elaborated by activated APCs and T cells and critical for the expansion of the immune response. The lack of costimulation during these interactions results in $\mathrm{T}$ cell anergy or apoptosis. Costimulation is not only important for the generation of adaptive immunity, but also is involved in the regulation of the various immune evasion mechanisms employed by cancer and chronic infections. Therefore, we hypothesized that costimulatory ligands may serve as the preferred adjuvants for generating effective and long-lasting adaptive immunity. We particularly focused on the natural costimulatory 
ligands of tumor necrosis factor (TNF) family given their pleiotropic function on cells of innate, adaptive, and regulatory immunity. The TNF family represents a critical group of costimulatory molecules since their receptors (TNFR) are inducibly expressed on activated cells and may serve as preferred targets for antigen specific responses through induction of expansion, survival of $\mathrm{T}$ cells and establishment of long term memory. Among these family members, 4-1BB/4-1BBL interaction has received the most attention as signaling through 4-1BB provides essential signals for $\mathrm{CD} 8^{+} \mathrm{T}$ cell expansion, effector function, and survival. Importantly, this signaling also endows effector $\mathrm{CD}^{+} \mathrm{T}$ cells resistant to suppression by regulatory $\mathrm{T}$ cells that are the predominant mechanism of immune evasion used by cancer and chronic infections.

Since 4-1BBL has costimulatory function as a cell surface membrane-bound protein and has no function in soluble form, our laboratory has previously generated a novel form of this molecule chimeric with streptavidin, SA-4-1BBL. This molecule was demonstrated to have robust costimulatory activity with a $\mathrm{Th}_{1}$ bias as a soluble protein. The main premise of this $\mathrm{PhD}$ thesis is to use $\mathrm{SA}-4-1 \mathrm{BBL}$ as an adjuvant platform to develop adjuvant systems for subunit vaccines with desired immune activities for targeted indications. We particularly focused on subunit vaccines against two indications; $Y$. pestis and breast cancer for the development of prophylactic and therapeutic vaccines, respectively. First, we tested if SA-4-1BBL can improve the immune efficacy of a lead subunit vaccine, $\mathrm{rF} 1-\mathrm{V}$ (a recombinant $Y$. pestis fusion protein), adjuvanted with alum with a $\mathrm{Th}_{2}$ bias against plague. Inasmuch as the lead candidate vaccine generates a $\mathrm{Th}_{2}$ response, and $\mathrm{Th}_{1}$ cellular responses have been shown to be important in protection against $Y$. pestis infection, we hypothesize that $\mathrm{SA}-4-1 \mathrm{BBL}$ as a $\mathrm{Th}_{1}$ adjuvant will 
improve the immune efficacy of the lead candidate vaccine. Single immunization with a vaccine formulation containing $\mathrm{rF} 1-\mathrm{V}$ as antigen and $\mathrm{SA}-4-1 \mathrm{BBL}$ as single adjuvant generated increased TNF $\alpha$ and IFN $\gamma$ signature cytokines for $\mathrm{Th}_{1}$ responses in both $\mathrm{CD} 4^{+}$ and $\mathrm{CD}^{+} \mathrm{T}$ cells without detectable antibody titers against $\mathrm{rF} 1-\mathrm{V}$. This vaccine formulation protected $20 \%$ of mice against bubonic plague. However, in a prime-boost setting, SA-4-1BBL and $\mathrm{rF} 1-\mathrm{V}$ generated long lasting high titers of antibodies and protected all mice from bubonic $Y$. pestis infection. Alum adjuvanted $\mathrm{rF} 1-\mathrm{V}$ vaccine generated high titers of antibodies against $\mathrm{rF} 1-\mathrm{V}$ without a significant $\mathrm{Th}_{1}$ response, and protected $80 \%$ of mice against bubonic plague. A combination of SA-4-1BBL and alum as an adjuvant system generated at balanced $\mathrm{Th}_{1}$ cellular and humoral responses that resulted in $100 \%$ protection in bubonic plague model.

Next, we tested if SA-4-1BBL has efficacy as adjuvant component of a Her-2/neu protein-based subunit vaccine against breast cancer and if the therapeutic efficacy of this subunit vaccine can further be improved by using toll-like receptor 4 (TLR4) agonist monophosphoryl lipid A (MPL) as adjuvant system. We hypothesize that MPL will work in synergy with SA-4-1BBL by targeting antigen presenting cell for activation, antigen presentation to $\mathrm{T}$ cells, leading to $\mathrm{T}$ cell activation and up regulation of 4-1BB receptor. Activated $\mathrm{T}$ cells will then serve as a direct target of SA-4-1BBL for expansion, acquisition of effector function, and establishment of long-term memory. A prime-boost immunization with extracellular domain of the rat Her-2/neu protein and SA-4-1BBL resulted in eradication of established Her-2/neu expressing A2L2 tumors in $10 \%$ of BALB/c mice. In contrast, MPL monotherapy did not have a therapeutic effect. However, vaccination with combined adjuvants resulted in eradication of established tumors in 30\% 
of BALB/c mice, and showed better therapeutic efficacy over individual therapies. Furthermore, immunization with combined adjuvants resulted in eradication A2L2 tumors in $20 \%$ of tolerogenic BALB/neuT mice. Depletion of Tregs prior to tumor challenge increased the efficacy of combined adjuvants to $40 \%$. The therapeutic efficacy of combined adjuvant platform correlated with increased tumor specific killing response and pro-inflammatory cytokine IFN $\gamma$ production. The combination of SA-4-1BBL and MPL achieved therapeutic efficacy in the absence of detectable toxicity as assessed by various indicators of toxicity, including liver enzymes, total number of various lymphocyte populations in several lymphoid tissues, vaccine-induced organ damage, and histological analysis of the liver.

Taken together, these data provide scientific rationale for using SA-4-1BBL as a novel adjuvant platform with other adjuvants having synergistic immune activities for the development of subunit vaccines against intracellular infections and cancer. 


\section{TABLE OF CONTENTS}

PAGE

ACKNOWLEDGEMENTS.....................................................

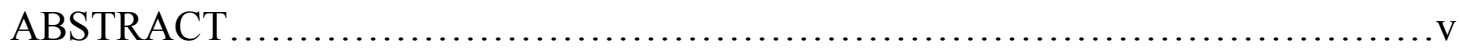

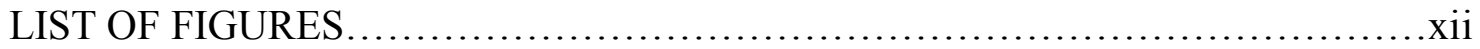

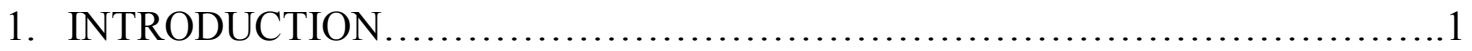

2. SA-4-1BBL AS A NOVEL ADJUVANT FOR PLAGUE VACCINE

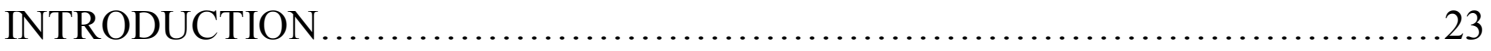

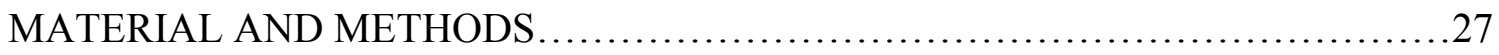

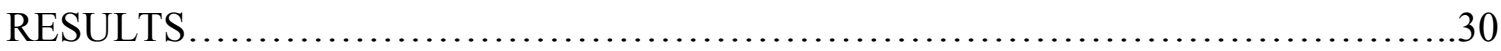

SA-4-1BBL and alum as adjuvant platform generates a $\mathrm{Th}_{1}$ response to the $\mathrm{rF} 1-\mathrm{V}$

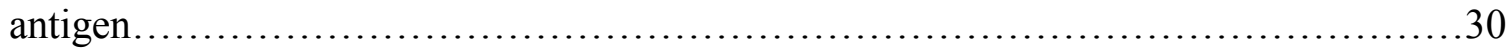

SA-4-1BBL and alum as adjuvant platform generates high antibody titers against $\mathrm{rF} 1-\mathrm{V}$ in a prime-only setting ...................................................

SA-4-1BBL and alum as adjuvant platform shifts the immune response towards $\mathrm{Th}_{1}$ direction in a prime-only setting........................................... 32

Vaccination with combination adjuvant platform provides protection against bubonic plague in a prime-only setting ............................................ 33

SA-4-1BBL generates a long lasting anti-rF1-V humoral response and protects mice from plague in a prime-boost setting............................................

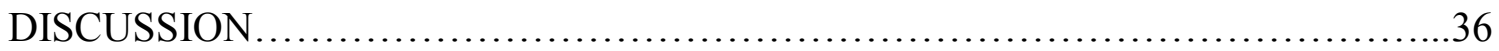

3. SA-4-1BBL AND MPL COMBINATION AS AN ADJUVANT SYSTEM FOR THE TREATMENT OF BREAST CANCER

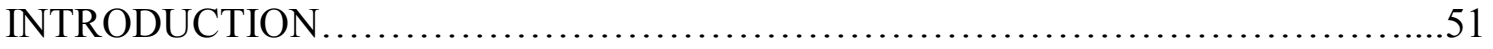

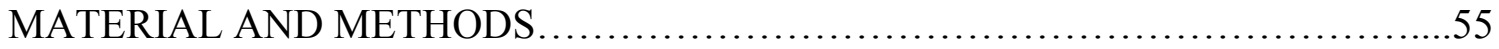


Expression and purification of recombinant rat Her-2/neu ECD .62

SA-4-1BBL adjuvanted rat Her-2/neu TAA-based vaccine induces elimination of established A2L2 tumors in BALB/c mice.

MPL induced antibody response against Her-2/neu does not correlate with survival.

Vaccination with combined adjuvants and rat Her-2/neu induces better anti-tumor killing response. 65

Vaccination with rat HER-2/neu + SA-4-1BBL induces robust antigen specific cytokine responses in $\mathrm{CD}^{+}$and $\mathrm{CD}^{+} \mathrm{T}$ cells 66

Vaccination with rat HER-2/neu + SA-4-1BBL increases the number of effector T cell populations in the dLNs of tumor bearing mice.

The therapeutic efficacy of SA-4-1BBL + MPL adjuvanted Her-2/neu vaccine is associated with the increased pro-inflammatory and decreased anti-inflammatory cytokine responses . .68

Combined adjuvant therapy with SA-4-1BBL and MPL generates a significant anti-tumor effect in tolerogenic BALB/neuT mice. 69

Therapeutic efficacy of the SA-4-1BBL + MPL adjuvant system is achieved in the absence of detectable toxicity .70

DISCUSSION .71

4. CONCLUSIONS AND FUTURE PROSPECTIVES .98

REFERENCES. 105

CURRICULUM VITAE 120 


\section{LIST OF FIGURES}

FIGURE

PAGE

1. SA-4-1BBL+Alum increases the cellular response of the plague vaccine...44

2. SA-4-1BBL + Alum induces antibody response for $\mathrm{rF} 1-\mathrm{V}$ in prime-only

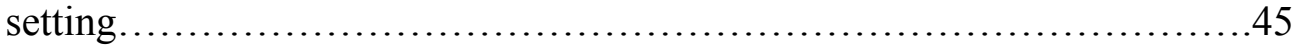

3. SA-4-1BBL + Alum shifts the cellular response towards $\mathrm{Th}_{1}$ in prime-only setting 46

4. One shot immunization with SA-4-1BBL + Alum protects mice from bubonic plague. 48

5. SA-4-1BBL generates a long-lasting anti-rF1-V humoral response in a primeboost setting. 49

6. SA-4-1BBL provides protection against bubonic plague with an $\mathrm{rF} 1-\mathrm{V}$ dose dependent manner.

7. Production of recombinant rat Her-2/neu ECD in bacteria .80

8. Production of recombinant rat Her-2/neu ECD in bacteria .81

9. Production of recombinant rat Her-2/neu ECD in Drosophila S2 cells, and Her-2/neu expression in A2L2 cells. ...

10. A prime-boost vaccination with SA-4-1BBL and MPL adjuvant system results in eradication of established A2L2 tumors.

11. A prime-boost vaccination with Her-2/neu + MPL induces significant antibody response

12. SA-4-1BBL + MPL adjuvant system induces a strong tumor specific killing response. .86

13. SA-4-1BBL based Her-2/neu vaccine induces a strong cytokine response...87

14. Vaccination with Her-2/neu + SA-4-1BBL induces high numbers of effector $T$ cell subsets in the dLNs of tumor bearing mice. 
15. Addition of MPL to the SA-4-1BBL adjuvanted vaccine increases proinflammatory and decreases anti-inflammatory cytokine production in the dLNs of tumor bearing mice

16. Verification of the expression of rat Her-2/neu gene in BALB/neuT mice with PCR.

17. While treatment with SA-4-1BBL and MPL adjuvanted vaccine results in eradication of established A2L2 tumors in $20 \%$ of BALB/neuT mice, depletion of Tregs further improves the vaccine efficacy

18. Treatment with SA-4-1BBL + MPL adjuvant system does not induce liver and

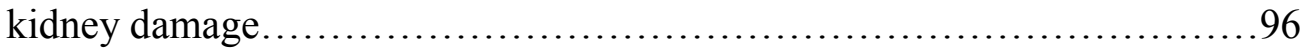

19. Treatment with SA-4-1BBL + MPL adjuvant system does not induce acute lymphocyte infiltration into the liver..................................97 


\section{CHAPTER 1}

\section{INTRODUCTION}

Successful vaccine development has significantly reduced the rate of highly infectious diseases and human mortality. As such, viral vaccinations against measles, rubella, and poliomyelitis dramatically reduced the incidence of these diseases. Further, vaccination for small-pox successfully eradicated small-pox, one of the most deadly diseases in the world, while poliomyelitis is in the verge of eradication [1]. These critical accomplishments in medicine demonstrate the importance of immune therapy. The tremendous impact of vaccination on humans was greatly demonstrated by vaccinologist Stanley Plotkin when he stated: "The impact of vaccination on human health of the world's peoples is hard to exaggerate. With the exception of safe water, no other modality, not even antibiotics, has had such a major effect on mortality reduction and population growth" [2]. While the development and worldwide use of such vaccines have had a dramatic impact on human health, still vaccines are not available for many infectious diseases and cancer.

The first human vaccine was developed against smallpox, and introduced by Edward Jenner in 1798 using related but weaker animal virus, cowpox [3]. However, the successful history of vaccination was started with Louis Pasteur when he first discovered 
the attenuation of gram negative bacteria, Pasteurella multocida in 1881. Subsequently, chemically attenuated rabies vaccine was developed for humans by Louis Pasteur and Emile Roux in 1885 [3], which was followed by the development of numerous attenuated and inactivated viral and bacterial vaccines. While these vaccine strategies were effective, possibly because inherently they contain natural adjuvants, such as particulated proteins, oligonucleotides, and lipids [4], there were concerns about stability during storage, occurrence of adverse effects and safety, especially in immunocompromised individuals [5]. Therefore, in the last several decades, as a result of advancement in molecular biology and genetic engineering, numerous strategies, such as DNA vaccines, viral or bacterial vector based vaccines, and recombinant protein/peptide vaccines have been employed to generate new human vaccines.

Naturally, in the early phase of infection, microbial compounds activate the innate arm of immunity through recognition by pattern recognition receptors (PRRs) on antigenpresenting cells (APCs). This leads to activation of APCs and induces the production of proinflammatory cytokines and upregulation of costimulatory molecules on the cell surface, which in turn primes the long-lasting adaptive immunity mediated by $\mathrm{T}$ and $\mathrm{B}$ lymphocytes [6]. This process provides T cells with three important signals, signals 1, 2, 3 [7] for their activation, acquisition of effector function, and long-term immune memory. Signal 1 is mediated by T-cell receptor (TCR), recognizing foreign peptides in the peptide binding groove of major histocompatibility complex (MHC) molecules on the surface of cells. Signal 2 is provided by various costimulatory molecules, while signal 3 is mediated by cytokines, such as IL-1 $\beta$, and IL-12 produced by activated APCs [8]. Failure to receive the second signal can lead to $\mathrm{T}$ cell anergy or apoptosis. As a result, an 
ideal vaccine should mimic the natural infection to generate a potent immune response with the induction of long-lived adaptive immune response.

Vaccine formulations should be chosen based on type of the treatment since prophylactic vaccines are given to healthy individuals to prevent infections and virally derived cancers. Generation of the long term immune memory, particularly humoral immunity, is the main goal. Otherwise, induction of an acute effector response may induce undesired side effects, such as inflammation, and may not provide protection for the future infections [9]. As a result, most of the traditional prophylactic vaccines against infections are targeting the generation of long-term antibody response. On the other hand, therapeutic vaccines are given to people with established infections and compromised immune system. Therapeutic vaccines primarily rely on $\mathrm{CD} 8^{+} \mathrm{T}$ cell responses for the elimination of viruses, such as HIV and herpes virus, intracellular bacteria, and cancer. Furthermore, generation of a long term memory is also critical for therapeutic tumor vaccines to control recurrences. For both prophylactic and therapeutic vaccines, induction of adaptive immunity which is mediated by $\mathrm{T}$ and $\mathrm{B}$ lymphocytes, and activation of innate immunity to shape the long-lasting adaptive immune response are crucial.

In addition to efficacy, some other criteria for new vaccine candidates for human use should be sufficed, such as; (1) safety, (2) stability, and (3) low cost for widespread use, especially for third world or developing countries. Finally, it is desirable that vaccine should not require frequent administrations [10]. Therefore, recombinant protein-based subunit vaccines containing well characterized single or a combination of proteins, fusion proteins, or peptides serving as antigens have been attractive strategies in the field of vaccinology in the last few decades since they can be administered safely and produced 
easily in a cost effective manner. However, protein-based subunit vaccines are poorly immunogenic and need to be administered with immune stimulant components known as adjuvants to generate an adequate and efficient appropriate immune response with longlived immune memory against target antigens $[11,12]$.

\section{Adjuvants:}

The first description of adjuvants (Latin word adjuvare, means "to help or aid") was made by Ramon as "substances used in combination with a specific antigen that produced a more robust immune response than the antigen alone" [13]. Adjuvants are a critical determinant of the success or failure of vaccines and the use of adjuvants as components of protein-based vaccine formulations can also reduce the amount of antigen needed for the generation of effective immunity and the number of vaccine administered. Because of the undefined nature of these adjuvants and their molecular complexity, it has been a challenge to fully understand mechanisms underlying their immune efficacy[5]. As a result, adjuvants have been described as "the immunologists dirty little secret" by Charlie Janeway.

Adjuvants are necessary to boost the immune response to naturally weak antigens. In 1920, the first adjuvants were developed and variety of substances, such as chemicals and microbial components, have been used to boost the immune system [14]. Adjuvant selection is made based on some parameters, including the physical and chemical nature of the antigen, type of desired immune responses, population age, and route of administration [4]. Historically, the importance of the humoral immune response for vaccine effectiveness has put the emphasis on the development of adjuvants, which are 
capable of enhancing antibody responses. As a result, many adjuvants are effective in increasing antibody titers, but do not elicit significant $\mathrm{Th}_{1}$ or cytotoxic $\mathrm{T}$ lymphocyte (CTL) responses [15]. However, for some diseases, such as malaria and tuberculosis, it is difficult to generate a broad antibody response to clear primary infection, and both humoral and cellular responses are required for protection from some viruses (e.g. HIV1) and intracellular bacteria (e.g. Y. pestis). Development of therapeutic cancer vaccines face an even greater challenge since their target often consists of tumor associated selfantigens (TAA) which are poorly immunogenic and expressed heterogeneously by tumor cells that undergo mutation. Although tolerance to TAAs is not absolute since Her-2/neu TAA generates antibody and CTL responses in some breast and ovarian cancer patients $[16,17]$, these responses are not potent enough to eliminate the tumor cells as tumors continue to grow and metastasize. Therefore, induction of potent cellular immunity is critical for the generation of therapeutic immune responses for chronic infections and cancer, and more importantly for the induction of long-term memory response to control tumor recurrences [18].

\section{Importance and role of vaccine adjuvants:}

$\mathrm{T}$ cell responses are critical for protection from cancer and infectious diseases, elimination of infected and tumor cells, and the induction of long lasting memory response to control recurrences. Moreover, induction of $\mathrm{T}$ helper $(\mathrm{Th})$ responses is also important to improve humoral and cellular response durability and quality. Recombinant protein antigens generate modest antibody responses while they induce little to no $\mathrm{T}$ cell responses when administered in the absence of adjuvants. As a result, adjuvants which 
are capable of inducing protective levels of humoral, CTL and Th cellular responses have been used in recombinant protein based vaccines.

Adjuvants can induce high antibody responses to vaccine antigens by increasing not only overall antibody titers but also functional antibodies [19]. In addition, adjuvants can also broaden antibody response, which is critical for the elimination of many pathogens that are capable of mutating their antigens (antigenic drift), such as malaria, influenza virus, HIV, and HPV. For example, it has been reported that in influenza and HPV vaccines, adjuvants broaden the antibody response to target antigens [20, 21]. Induction of strong CTL responses has also been observed against target antigens using adjuvants [22]. As such, immunization with immunostimulating complexes (ISCOMs) and HIV-1gp160 envelope glycoprotein or influenza haemagglutinin generates long lasting antigen specific $\mathrm{CD}^{+} \mathrm{T}$ cell reponses [23]. Further, QS-21 (saponin based adjuvant) has also been shown to induce CTL responses to subunit viral antigens such as HIV-1 gp120, and human cytomegalovirus gB, and induced neutralization of the respiratory syncytial virus (RSV) by heightening $\mathrm{Th}_{1}$ driven $\mathrm{IgG}_{2 \mathrm{a}}$ antibody titers to virus fusion (F) protein [24].

Recombinant protein based sub-unit vaccines may require multiple administrations to elicit sufficient humoral immune response. Recruitment of adjuvants may reduce the number of immunizations or the amount of antigen used in the vaccine formulation. As such, it has been demonstrated that immunization with glucopyranosyl lipid adjuvant-stable emulsion (GLA-SE) and recombinant influenza H5 protein reduced the amount of antigen required for the generation of proper antibody response after one immunization more than 30-fold compared to antigen alone [25]. 


\section{Currently FDA approved or adjuvants under development}

With the development of recombinant vaccines (particularly recombinant protein based vaccines) that are less immunogenic, the investigation and design of new adjuvants have been the object of industry and academic in the last few decades. However, development of new adjuvants has been challenging due to their molecular complexity and lack of significant insight in their mechanisms of action [5]. The idea of using inactivated or live attenuated microbes in vaccine formulations is primarily based on their ability to activate innate immunity through activation of APCs, and presentation of exogenous antigens to $\mathrm{T}$ cells. As a result, several adjuvant candidates have been under development, such as oil emulsions, cytokines, or saponin-based adjuvants, and mineral salts such as aluminum hydroxide (alum) and less toxic version of bacterial lipopolysaccharide (LPS), monophosphoryl lipid A (MPL), have already been approved by FDA. The adjuvants target primarily innate immunity through activation of APCs and uptake and presentation of antigens by these cells to T cells [26]. Finally, activation of innate immunity leads to the prime of adaptive immune responses where cellular arm plays an important role for clearance of infected and transformed cancer cells, while humoral arm clears pathogens through antibodies.

Adjuvants can be divided into three major groups according to their mechanisms of action, such as delivery vehicles, immunomodulators, and both delivery and immunomodulatory components [4]. Delivery vehicles do not contain immune stimulatory molecules, and instead they induce the desired immune response through (1) sustained release of antigen by forming an antigen depot and (2) increasing antigen uptake and presentation by APCs to increase the specific immune response to the antigen. 
Vehicles include mineral salts, emulsions, liposomes or virosomes. On the other hand, immunomodulatories directly act on the immune system by (1) up-regulation of cytokines and chemokines, (2) recruitment of specific cell types to the inoculation site, (3) activation and maturation of APCs by induction of the costimulatory molecule expression and signaling, and (4) activation of inflammasomes [13, 27]. Immunomodulatories include saponins, cytokines, TLR ligands, and costimulatory molecules. Vehicles which also deliver immunostimulatory components are in the third group such as mineral salts, emulsions, and immunostimulating complexes (ISCOM) [28].

\section{Delivery vehicles}

\section{Aluminum based adjuvants:}

In human vaccines, the most widely used adjuvants are aluminum based adjuvants and FDA approved Alhydrogel was the first and only adjuvant until MPL approval in the context of the prophylactic HPV vaccine in 2009. Aluminum hydroxide or phosphate adjuvants are currently being used in Diphtheria, Tetanus, HBV and HPV vaccines. Alum acts by inducing the formation of an antigen depot at the injection site which has been shown to be critical in alum adjuvanticity [13] and slowly releases antigen to stimulate the immune system continuously, and enhance the antigen uptake [29, 30].

Alum is a potent adjuvant for the generation of antibody responses to target antigens and primarily induces $\mathrm{Th}_{2}$ responses $\left(\operatorname{IgG}_{1}, \operatorname{IgE}\right.$ and the production of IL-4), rather than $\mathrm{Th}_{1}$ responses in many of the traditional childhood vaccines against bacterial or viral surface molecules [29-31]. Since the induction of cellular immunity is critical for protection from cancer and infection, new vaccine approaches utilize another adjuvant, 
MPL, along with alum (e.g., AS04) to induce the generation of cellular immunity, particularly $\mathrm{Th}_{1}$, for optimal effectiveness [32]. Although alum based adjuvants have been shown to be efficacious, they are associated with severe inflammatory site reactions due to recruitment of blood cells, formation of granulomas, and increased allergenicity due to high IgE titers. Moreover, aluminum based adjuvants cannot be frozen and lyophilised [33, 34], limiting their widespread use.

\section{Oil in water emulsions:}

Particulate adjuvants, such as oil-in-water emulsions, are naturally utilized for uptake by APCs to induce potent immune responses. MF59 is the first oil-in-water adjuvant licensed for use in Europe for seasonal and pandemic influenza (H1N1 and H5N1) vaccines. It consists of the oil squalene encircled by non-ionic surfactants. Fluad, a subunit influenza vaccine containing MF59 licensed in Europe by Novartis [35], has been distributed as more than 27 million doses since 1997 [36] and has shown to provide strong memory and sustained antibody responses compared to the non-adjuvanted vaccine. Aflunov is another influenza vaccine containing MF59 licensed by Novartis and marketed in 2010 to protect against H5N1 flu. MF59 has also been evaluated as an adjuvant in vaccines for other viral infections, such as HIV, hepatitis C, and cytomegalovirus.

MF59 generates its immune stimulatory activity by the induction of an inflammatory environment at the inoculation site characterized by enhanced cytokine and chemokine responses, which in turn induce the trafficking of $\mathrm{CD}_{1} 1 \mathrm{~b}^{+}$and $\mathrm{MHC} \mathrm{II}^{+}$cells to the muscle [37]. Moreover, it is demonstrated that it targets macrophages and DCs at the inoculation site and draining lymph nodes, enhancing antigen uptake by forming 
antigen depot [38], increasing antibody production against target antigen, and favoring a $\mathrm{Th}_{2}$ response [39]. Although, oil in water emulsions were associated with generation of autoantibodies in non-autoimmune mice [40], MF59 adjuvanted influenza vaccines did not induce autoantibodies. However, emulsion adjuvants may induce side effects, such as inflammatory reactions, granulomas and ulceration at the inoculation site.

\section{Immunomodulators}

\section{Saponin based adjuvants:}

Saponins are natural glycosides of steroid or triterpene found in plants, lower marine animals and some bacteria [41]. They have the ability to modulate immune responses by different action mechanisms, such as antitumor, antiviral, antifungal, and anti-inflammatory [42]. They can induce not only antibody responses, but also cellular immune responses against target antigen with the advantage of the requirement of low doses needed for adjuvanticity. It has been demonstrated that saponins induce production of $\mathrm{Th}_{1}$ cytokines (IL-2 and IFN- $\gamma$ ) and antibodies of the $\operatorname{IgG}_{2 \mathrm{a}}$ isotype, and they may interact with APCs to modulate immune responses [22, 43]. Most likely, saponins perform these stimulatory activities through the interaction with APCs. Although it is not known yet, the adjuvant effect of saponins might be related to their ability to induce pore formation. It is also likely that pore formation induces antigen uptake and presentation and subsequent activation of CTL responses [44].

The lead candidate saponin based adjuvants are Quil A and its derivative QS-21. QS-21 has been tested as an adjuvant initially for cancer vaccines (melanoma, prostate, and breast) and subsequently for infectious diseases (malaria, herpes, HIV-1, influenza, or hepatitis B) in clinical trials and showed promise [24]. Further, QS-21 adjuvant is also 
being tested in ACC-001 vaccine for mild to moderate Alzheimer's disease in a clinical trial conducted by Pfizer since 2009 and in sialyl Lewis-keyhole limpet hemocyanin conjugate vaccine for metastatic breast cancer by NCI since 2007 . The critical advantage of the use of Quil A and QS-21 in vaccine formulations is that they stimulate both $\mathrm{Th}_{1}$ and CTL responses against vaccine antigens $[23,45,46]$. However, a balance of efficacy versus side effects is the key for these adjuvants since Quil A and QS-21 are highly toxic and have hemolytic activity due to their structure and cause hemolysis of the red blood cells which restricts their use in human vaccines [47].

\section{Cytokines:}

Cytokines are naturally derived substances that play an important role in controlling the immune system. They can improve both humoral and cellular immune responses by inducing antibody production, proliferation and differentiation of $\mathrm{B}$ and $\mathrm{T}$ cells, enhancing cytokine production and improving cytotoxicity activity of T cells [48]. IL-2 has been extensively studied as an adjuvant for vaccines due to its pleiotropic effects and critical role in T-cell responses. IL-2 is the first cytokine administered in clinical trials in cancer vaccines. However, its action mechanism depends on the form of administration. Continuous IL-2 administration increase the proliferation of antigen specific $\mathrm{T}$ cells and IL-2 in oil-in-water emulsion induces antibody response [49]. Although IL-2 has been used as an adjuvant in clinical trials for melanoma [50-54], it has been reported by NCI that melanoma vaccines given with IL-2 do not generate better anti-tumor responses than IL-2 alone [55]. However, dosing and timing of IL-2 administration still need to be well determined. Even though high doses or multiple administrations of IL-2 induce better clinical outcome, at high doses, the use of IL-2 is 
associated with toxicity [56]. Furthermore, while IL-2 enhances cytotoxic activity of NK cells and CTLs, it can also induce the expansion of $\mathrm{CD} 4^{+} \mathrm{CD} 25^{+} \mathrm{Foxp}^{+}$regulatory $\mathrm{T}$ cells (Tregs), which in turn contribute to tumor immune suppressive activity [57].

There are other cytokines, such as IL-7 and IL-21, have been tested in clinical trials against cancer. IL-7 does not stimulate expansion of Tregs or induce toxicity like IL-2, but it did not generate an anti-tumor effect either [58-60]. On the other hand, IL-21 showed anti-tumor activity [61], but it also has some limitations due to the expression of its receptor on multiple tumor cells $[62,63]$. IL-15 is another cytokine under development by NCI to be tested in clinical trials [56].

\section{Toll like Receptor (TLR) agonists:}

Understanding the recognition of microbial structures by innate immunity and its important role in host defense against infection has showed rapid progress. Due to the critical role of TLR signaling in innate immunity and bridging innate immunity with adaptive immune responses, several well characterized and defined TLR ligands have been developed as adjuvants in human vaccines. TLRs are a family of pattern recognition receptors (PRR) which recognize the pathogen associated molecular patterns (PAMP), such as proteins, lipids, lipoproteins, and nucleic acids of microbes. TLR ligation induces the activation of APCs, and the production and release of proinflammatory cytokines, such as TNF, IL-1, IL-6, which in turn prime and activate antigen specific T cells.

Among all the members in this family, TLR4 agonists are the most developed adjuvants. TLR4 responds to LPS, an outer membrane component of Gram-negative bacteria. MPL, a derivative of LPS from Salmonella Minnesota, is the second adjuvant to be approved by FDA in the USA. It is currently used as one of the components of the 
adjuvant system in the Cervarix (HPV) and Fendrix (HBV) vaccines from GSK along with L1 recombinant protein and hepatitis B surface antigen (HBsAg).

The TLR7/8 pathway plays an important role against viral infections which recognizes single stranded RNAs. TLR7 stimulation induces production of IFN $\alpha$, while TLR8 stimulation leads to production of proinflammatory cytokines through NF-kB activation. TLR7 agonist imiquimod has been approved by FDA for topical administrations for the treatment of basal cell carcinoma (BCC) and actinic keratosis (AK) [27]. However, TLR7/8 agonist, resiquimod, has shown inconsistent results in clinical trials for genital herpes simplex virus (HSV)-2 infection [64].

TLR9 also plays an important role for recognition of pathogens and signaling through TLR9 leads to secretion of pro-inflammatory cytokines. TLR9 agonist CpG oligodeoxynucleotide (ODN) 1018 is the adjuvant in the Heplisav (Dynavax) vaccine candidate for HBV, which has completed a phase III clinical trial. It has been shown that Heplisav induces rapid and increased antibody production and sustains the antibody response in healthy adults [65]. It was recently announced that another TLR9 agonist adjuvant called IC31 is currently undergoing Phase I/IIa clinical trial for a candidate tuberculosis vaccine. In preclinical studies, it has been demonstrated that IC31 promotes highly efficient $\mathrm{Th}_{1}$ responses through MyD88 signaling pathway [66].

Nevertheless, inappropriate TLR signaling may result in acute and chronic inflammation, and systemic autoimmune diseases, even though it is giving promising results in the clinic. Defects in the negative regulation of TLR signaling lead to development of such diseases. Furthermore, endogenous compounds released by dying 
cells can stimulate TLR signaling, and contribute to inflammation and autoimmune diseases [67].

\section{Costimulatory molecules:}

For the generation of protective immune responses through vaccination, it is critical to activate antigen specific $\mathrm{T}$ cells. $\mathrm{T}$ cell activation is regulated by successive signaling events from first signal, TCR/MHC interaction; second signal, costimulatory molecules, and third signal, proinflammatory cytokines [8]. Failure to receive second signal can lead to $\mathrm{T}$ cell anergy or apoptosis. In early phase of natural infections, microbial compounds are recognized by PRRs on the APCs, which lead to generation of second and third signals for $\mathrm{T}$ cell activation [7]. However, these signals can also be provided by vaccines with the recruitment of adjuvants such as costimulatory molecules [68]. Costimulatory receptors could be divided into two super families; immunoglobulin family (IgSF) and tumor necrosis factor receptor family (TNFRSF). It has been proposed that for initial $\mathrm{T}$ cell activation signaling from IgSF is important, while TNFRSF costimulation is critical for T cell effector function, survival and memory $[69,70]$.

Use of costimulatory molecules targeting activation and effector function of $\mathrm{T}$ cell responses in vaccines is divided into two approaches, those enhance the antigen specific immune response or shape it for desired immunity, and others to block or remove negative regulatory mechanisms to generate potent immune responses [71]. In the case of poorly immunogenic tumors, such as B16 melanoma, both approaches have been utilized to generate better anti-tumor responses. For example, co-administration of agonistic antibody for 4-1BB receptor in TNFRF and blocking antibody for CTLA-4 in IgF along with B16-Flt3-ligand vaccine (FVAX), promoted B16 melanoma tumor rejection in a 
synergistic manner [72]. Moreover, combined treatment with anti-DR5, anti-4-1BB, and anti-CD40 mAbs successfully eradicated established 4T1 mammary tumors [73].

\section{Immunoglobulin superfamily:}

In IgSF, perhaps the most well characterized and described members are CD28 and B7 families. The CD28 glycoprotein is constitutively expressed on naive CD4 ${ }^{+}$and $\mathrm{CD}^{+} \mathrm{T}$ cells, while its ligands B7-1 and B7-2 are inducibly expressed on the surface of APCs upon activation. CD28 ligation with B7-1 and B7-2 molecules provides essential signal for $\mathrm{T}$ cell activation, expansion and differentiation. Therefore, tumor cells transfected with B7 family members have been used in cancer immunotherapy to promote them to act as APCs and induce T cell activation $[74,75]$. Moreover, CD28/B7 interaction prevents apoptosis of $\mathrm{T}$ cells and sustains their proliferation, which was determined by the upregulation of bcl- $\mathrm{x}_{\mathrm{L}}$ gene expression [76].

The inhibitory receptor cytotoxic T lymphocyte antigen 4 (CTLA-4) expression is induced following $\mathrm{T}$ cell activation and subsequent to CTLA-4 upregulation, CD28 expression is downregulated. While the B7/CD28 interaction positively stimulates $\mathrm{T}$ cell responses, B7/CTLA-4 ligation negatively regulates these responses. To overcome the inhibitory mechanism of CTLA-4 for T cell responses which is critical for elimination of cancer cells and pathogen infected cells, antibodies targeting CTLA-4 have been developed. As such, Ipilimumab (Yervoy), a humanized monoclonal antibody (mAb), was developed by Bristol-Myers Squibb and approved by FDA in 2011 for the treatment of melanoma. A fusion protein consisting $\mathrm{Fc}$ region of $\mathrm{IgG}_{1}$ and extracellular domain of CTLA-4 targeting B7 molecules on APCs, called Abatacept (Orencia), was also developed by Bristol-Myers Squibb and licensed in the US for the treatment of 
rheumatoid arthritis [77]. Furthermore, another fusion protein called Belatacept, which differs from Abatacept with only two aminoacids, has also been approved by the FDA in 2011 to provide engraftment in transplantation [78]. The mechanism of these fusion proteins is to prevent $\mathrm{T}$ cell activation by binding to $\mathrm{B} 7$ molecules and blocking $\mathrm{CD} 28$ mediated co-stimulatory signaling to $\mathrm{T}$ cells.

Employment of the molecules targeting IgSF members has the common goal of targeting APCs, as a subsequent event they either induce or prevent $\mathrm{T}$ cell activation. Moreover, cytokines such as IL-2 and IFN- $\gamma$ have also been utilized to target T cells by inducing their proliferation and responsiveness to the signals generated by APCs in response to antigen, but these treatments showed limited efficacy $[79,80]$. Therefore, an alternative approach to directly target activated $\mathrm{T}$ cells is the use of TNFRSF members, due to their expression on only activated $\mathrm{T}$ cells and their important roles in the activation, acquisition of effector function, and establishment of long-term $\mathrm{T}$ cell memory.

\section{TNF receptor superfamily:}

Development of vaccines which are capable of inducing $\mathrm{T}$ cell responses, especially CTLs, is critical for infectious diseases and cancer. Regulation of Treg suppressive function is another important aim for immunotherapy. While in the case of autoimmune or inflammatory diseases, enhancing Treg numbers or activity is beneficial; in cancer or infectious diseases decreasing Treg numbers and suppressing their function is essential for the therapy [70]. In this context, TNFRSF play important roles in regulating both adaptive and regulatory immunity. Moreover, these receptors are inducibly expressed on activated $\mathrm{T}$ cells upon antigen stimulation, unlike CD28, which 
gives another advantage to serve as adjuvant candidates for vaccines to generate antigenspecific immune responses [70]. Importantly, the expression of TNF ligands on the APCs are also induced after the receiving innate immune signals, such as TLRs, and adaptive signals, such as IFN- $\gamma$.

Targeting TNFR/TNFL interaction could be divided into two strategies: (1) Blocking these interactions to suppress the pathogenic immune responses in autoimmune and inflammatory diseases and (2) augmenting the signaling generated by TNFR ligation to stimulate the protective immune responses in cancer.

Different types of therapies, such as gene therapy, recombinant molecules, antibodies and Fc fusion proteins containing the TNFRSF ectodomains, targeting TNF ligands or TNFRSF members have been tested in the clinic and approved for human use. Infliximab (Remicade; Centocor Ortho Biotech), a chimeric TNF-specific antagonistic antibody, was the first drug approved in 1998 to treat RA, Crohn's disease, and ulcerative colitis [81]. Adalimumab was the first fully human TNF-specific antagonistic antibody approved in 2002 to treat RA, JIA, psoriatic arthritis, psoriasis, and Crohn's disease [81]. In the case of cancer, agonistic therapies and depleting antibodies have been evaluated in clinical trials. Tasonermin, a recombinant TNF molecule, and Brentuximab, depleting antibody specific for CD30 molecule have been approved for the treatment of sarcoma and Hodgkin's lymphoma, respectively [81]. Other agonistic antibodies targeting the members of TNFRSF, such as CD40, OX40, or 4-1BB, have been tested in clinical trials for cancer, but the main concern with the use of such antibodies is the potential of deleterious side-effects $[82,83]$.

\section{Adjuvant systems:}


The combination of immunostimulatory components in different adjuvant systems have been developed to promote appropriate protective and therapeutic immune responses generated by vaccination. When alum adjuvanted Gardasil vaccine and alum + MPL adjuvanted Cervarix vaccine were compared, it has been reported that Cervarix treatment resulted in significant increase in the neutralizing antibody titers for HPV-16 compared to Gardasil [84], which displays the contribution of MPL and importance of combining adjuvants with different action mechanisms.

MPL + alum is present in AS04, and MPL + QS-21 is present in AS01 (liposome based) and AS02 (emulsion based) adjuvant systems [85]. AS04 adjuvant system is a part of hepatitis B vaccine called Fendrix (GSK) and HPV vaccine: Cervarix (GSK). AS04 adjuvant system directly activates the innate immunity by activation of NF-kB and induction of cytokine production, which in turn, induces the activation of antigen specific adaptive immune response [6]. Although synergistic effect of alum and MPL has not been noted, it has been suggested that alum prolongs the cytokine response of AS04 at the injection site [6].

AS01 and AS02 adjuvanted candidate malaria vaccines consist of two proteins, RTS (a sporozoite surface antigen of P. falciparum) and S (surface antigen of Hepatitis B virus). Although there are no AS01 and AS02 adjuvanted vaccines licensed yet, they have been studied in clinical trials. GSK's RTS, S vaccine candidate for malaria containing AS-01 adjuvant system has reduced the clinical malaria cases up to $46 \%$ in children and $27 \%$ in infants in a phase III clinical trial.

AS03 is another adjuvant system composed of squalene, $\alpha$-tocopherol, and polysorbate 80 in an oil-in-water emulsion similar to MF59, has been licensed for H1N1 
influenza vaccine, Pandemrix (GSK), in Europe for 2009 H1N1 pandemic flu [84]. Recently, AS03 adjuvanted H5N1 influenza vaccine Q-pan (GSK) has been approved by FDA. On the other hand, Pandemrix was discontinued in Europe due to an increased risk of narcolepsy found following vaccination. It has been shown that AS03 activates the innate immunity and enhances antigen uptake and presentation by APCs in draining lymph nodes, which in turn, activates adaptive immune responses, principally antibody response and immune memory [86].

Generation of potent $\mathrm{T}$ cell responses against tumor and infection is critical for the elimination of transformed and infected cells. In the case of infection where the generation of neutralizing antibodies is not potent enough to block the infection and cancer where TAAs induce host tolerance, cellular immunity comprising $\mathrm{Th}_{1}$ and CTL responses is important. It is possible that the success of recombinant protein based vaccines, such as HBV and HPV, was because of the use of adjuvants (AS04 adjuvant system) with a distinct mechanism of action and targeting different immune cells for activation $[10,87]$. Therefore for most of the infectious diseases and cancer vaccines, development of adjuvant systems which can induce both $\mathrm{T}$ cell, especially $\mathrm{CD} 8^{+} \mathrm{T}$ cells, and antibody responses is an important goal. In the case of enhancement of $\mathrm{T}$ cell responses, cytokines, TLR ligands and costimulatory molecules have been used as adjuvants in vaccine formulations.

\section{Costimulatory SA-4-1BBL as an adjuvant platform for development of vaccines:}

Although development of adjuvants targeting $\mathrm{T}$ cell stimulation is an important goal, in the case of therapeutic cancer vaccines, regulation of immune suppressive mechanisms is another important aim for immunotherapy since tumor cells employ these 
mechanisms to evade immune attack. Not only tumor cells, but also viruses, such as hepatitis B virus, herpes, influenza, and retroviruses, exploit Tregs to evade immune responses. Therefore, strategies targeting the trafficking of Tregs to the site of immunization by small molecule antagonists have been explored $[88,89]$.

Among all the members in TNFSR family, targeting 4-1BB has been having growing interest due to its pleiotropic effects on cells of innate, adaptive, and regulatory immunity [90-94]. Its expression on the activated $\mathrm{CD}^{+} \mathrm{T}, \mathrm{CD} 8^{+} \mathrm{T}, \mathrm{NK}$ and $\mathrm{NK} \mathrm{T}$ cells provides another advantage to target activated cells of innate and adaptive immunity, as such antigen specific CTL have been selected based on 4-1-BB expression [95]. 4-1BB signaling has been shown to be critical for $\mathrm{T}$ cell expansion, survival, acquisition of effector function and establishment of long-term memory [96, 97]. Additionally, we have previously reported that $4-1 \mathrm{BB}$ is constitutively expressed on a subset of DCs which may heighten antigen uptake and cross-presentation to $\mathrm{T}$ cells [98]. Ligation of 4-1BB has been shown to inhibit Treg suppressive activity in vivo and in vitro [93] through a direct effect by blocking Treg suppressive function or an indirect effect by rendering effector $\mathrm{T}$ cells resistant to Treg suppression [94].

In clinical studies, agonistic human anti-4-1BB antibodies have been evaluated either alone or in the combination with rituximab (anti-CD20 antibody) to treat melanoma (BMS-663513; Phase I/II) and non-Hodgkin's lymphoma (PF-05082566; Phase I), respectively. BMS-663513 mAb is also being used to treat a number of different solid tumors including renal cell carcinoma, ovarian carcinoma, and non-small cell lung carcinoma either as a single agent or in combination with chemotherapy, radiotherapy, or both [99]. However, liver toxicity was reported in the melanoma patients, which might be 
because of nonspecific or excessive NK cell activity in liver, or accumulation of CTLs into the liver [81]. Toxicity issues may impede future studies and lead to termination of studies using agonistic antibodies.

To overcome toxicity problems alternative strategies should be developed to target 4-1BB. For this purpose, we have focused to generate a novel form of 4-1BB ligand which is functional in soluble form and without having toxicity. Natural 4-1BBL is a cell membrane-bound protein and has no activity in soluble form [100]. Therefore, we generated a novel form of this ligand composed of the extracellular domain of murine 41BBL fused to the C-terminus of a modified core-streptavidin (SA-4-1BBL) [101]. The SA domain allows the formation of tetramers and higher structures that have the ability to crosslink the 4-1BB receptor on immune cells for effective signaling [98, 102]. More importantly, this chimeric molecule has better costimulatory activity than an agonistic anti-4-1BB antibody without antibody associated severe toxicity [103]. The activity of this natural SA-4-1BBL has been established in our lab in various preclinical tumor models, and demonstrated that SA-4-1BBL adjuvanted recombinant protein or peptidebased vaccines generate robust $\mathrm{Th}_{1}$ and CTL responses with therapeutic efficacy [98, 102, 104-107]. More importantly, we have reported that SA-4-1BBL inhibits the formation of inducible Tregs and confers $\mathrm{CD} 4^{+}$and $\mathrm{CD} 8^{+} \mathrm{T}$ effector cells refractory to Treg suppressive activity [106]. Based on our previous observations, we sought to investigate if SA-4-1BBL can be utilized as a novel adjuvant for plague vaccine in an infection model, a tolerant tumor model with a self TAA, and if the efficacy of SA-41BBL can be further improved by addition of other immune modulators to the vaccine formulation. Therefore, the first major goal of this Ph.D. dissertation was to test the SA- 
4-1BBL efficacy as monotherapy and in combination with alum for bubonic plague infection and determine the mechanistic insights of the vaccine efficacy. The second goal was to determine the therapeutic efficacy of SA-4-1BBL and MPL adjuvanted Her-2/neu based protein vaccine in A2L2 breast cancer model and to evaluate the mechanisms behind the efficacy. 


\section{CHAPTER 2}

\section{SA-4-1BBL AS A NOVEL ADJUVANT FOR PLAGUE VACCINE}

\section{Introduction}

Yersinia pestis is a Gram-negative facultative intracellular bacterium which causes bubonic and pneumonic plague and has a potential to be used as a bioweapon [108-110]. Currently there is no FDA approved vaccine available to protect the public from a potential epidemic or bioterrorism event. While $Y$. pestis infection can be treated with antibiotics, the effective treatment window for primary pneumonic infection is very short (less than 24 hours after exposure) [111]. Furthermore, naturally acquired resistance to antibiotics has been reported [112], and weaponized $Y$. pestis could likely be modified to be resistant to antibiotic treatment. Therefore, development of novel vaccines with protective efficacy against plague is imperative.

Historically, plague vaccines were made from live-attenuated or inactivated forms of the microbe. While these vaccines were effective against plague, there were concerns about stability during storage, occurrence of adverse effects and safety, especially in immunocompromised individuals [113]. Advancement in recombinant DNA technology allowed for the development of subunit vaccines containing well-characterized proteins serving as antigens. Such subunit vaccines overcome various issues related to the use of complete pathogens as vaccine. However, protein-based subunit vaccines are poorly immunogenic and need to be administered with immune-stimulant components known as 
adjuvants $[11,12]$. Although various $Y$. pestis antigens have been assessed for the development of subunit vaccines, the $17 \mathrm{kDa} F 1$ extracellular capsule protein and the 37 $\mathrm{kDa}$ LcrV virulent factor are currently considered the most promising antigens [114]. Immunization with a mixture of F1 and LcrV antigens provided protection against plague $[113,115,116]$. The current lead candidate subunit vaccine for plague is based on a recombinant fusion protein between $\mathrm{F} 1$ and $\mathrm{LcrV}$ called $\mathrm{rF} 1-\mathrm{V}$. This vaccine is adjuvanted with alum and its protective efficacy against plague has been shown in rodent, guinea-pig, and non-human primate (NHP) models [117, 118], although in cynomolgus macaques it was protective $80-100 \%$, studies in African green monkeys were not satisfactory enough [119], and it is currently under clinical evaluation by the US Department of Defense to protect military personnel to aerosolized Y. pestis [120]. However, the inconsistent efficacy in NHP raises a significant concern for the protective efficacy in humans and indicates the need for further improvement of this subunit vaccine.

The protection in rodents correlated with the generation of high titers of antibodies against $\mathrm{rF} 1-\mathrm{V}[121,122]$. Indeed, passive transfer of antibodies was effective in protecting mice against plague $[121,123,124]$. However, studies in nonhuman primate models have not been as clear [125][126]. For example, while cynomolgus macaques consistently generated high titers of antibodies against both the F1 and LcrV antigens when immunized with the $\mathrm{rF} 1-\mathrm{V}$ vaccine that correlated with protection, African green monkeys generated much lower titers against F1 (though consistent), demonstrated high variability in anti-LcrV antibody titers, and did not show a correlation between antibody 
titers and protection [127]. Importantly, animals that failed protection had bacteria reservoir, suggesting antibodies failed to kill bacteria and disease progressed [128].

On the other hand, the $\mathrm{Th}_{1}$ cytokines TNF- $\alpha$ and IFN- $\gamma$ also appear to play a requisite role for protection against $Y$. pestis infection [129-132]. As such, exogenous administration of IFN- $\gamma$ and TNF- $\alpha$ protects naïve mice against $Y$. pestis infection [132], and neutralization of either cytokines abrogates the protection in actively vaccinated mice [129]. Furthermore, IFN- $\gamma$ and TNF- $\alpha$ also contribute to protection mediated by passive serum transfer or administration of $\mathrm{F} 1$ or LcrV specific mAbs which is demonstrated by abrogated efficacy in IFN- $\gamma \mathrm{R}$ - and TNF- $\alpha$-deficient mice and neutralization of these cytokines $[129,130]$. Contribution of cellular immunity to plague vaccine has further been demonstrated by adoptive transfer of $Y$. pestis-primed $\mathrm{T}$ cells to naive B-celldeficient $\mu \mathrm{MT}$ mice. This resulted in protection against lethal $Y$. pestis challenge. Moreover, treatment of $\mu \mathrm{MT}$ mice with T-cell-depleting mAbs resulted in abrogation of protection conferred by vaccination with live $Y$. pestis [133]. Given the demonstrated role of $\mathrm{Th}_{1}$ responses in protection against plague, one potential way to improve the efficacy of the $\mathrm{rF} 1-\mathrm{V}$ plague vaccine would be to increase the $\mathrm{Th}_{1}$ response by altering the adjuvant system.

Although alum, as the adjuvant component of the lead $\mathrm{rF} 1-\mathrm{V}$ vaccine, generates robust antigen-specific $\mathrm{Th}_{2}$ antibody responses, its efficacy in generating $\mathrm{Th}_{1}$ responses is minimal [29]. Inasmuch $\mathrm{Th}_{1}$ responses play a critical role in long term immune memory and clearance of intracellular pathogens, many subunit vaccines, including $\mathrm{rF} 1-\mathrm{V}$, would likely benefit from a more balanced $\mathrm{Th}_{1} / \mathrm{Th}_{2}$ responses. Signaling through the costimulatory 4-1BB receptor of the TNFR superfamily is critical for the generation of 
adaptive $\mathrm{Th}_{1}$ and CTL responses and establishment of long-term immune memory [98, $102,104]$. Therefore, we have recently hypothesized that agonists of 4-1BB receptor may serve as an effective adjuvant with a $\mathrm{Th}_{1}$ bias. Inasmuch as the natural ligand, $4-1 \mathrm{BBL}$, functions as a cell-surface protein and has no activity in soluble form, we generated a chimeric form of this agonist (SA-4-1BBL) by fusing its functional extracellular domain to a modified form of core streptavidin $[98,102,134,135]$. SA-4-1BBL activates DCs and $\mathrm{T}$ cells to generate a robust antigen-specific $\mathrm{Th}_{1}$ response with demonstrated therapeutic efficacy in several preclinical cancer models $[98,102,136]$.

Given that both humoral and $\mathrm{Th}_{1}$ cell mediated immune responses are important for the control/clearance of $Y$. pestis, we herein assessed the efficacy of SA-4-1BBL and alum as an adjuvant system to produce a balanced $\mathrm{Th}_{1} / \mathrm{Th}_{2}$ immune response in a short term prime-only setting and the efficacy of SA-4-1BBL alone to generate humoral response in a long term prime-boost setting. In the short term study, when we compared SA-4-1BBL and alum, SA-4-1BBL generated robust $\mathrm{Th}_{1}$ cellular responses against $\mathrm{rF} 1-\mathrm{V}$ without a significant antibody response while in combination with alum, SA-4-1BBL generated a mixed $\mathrm{Th}_{1}$ and $\mathrm{Th}_{2}$ cellular response without a dramatic positive or negative impact on the titers of anti-rF1-V antibodies generated by alum alone. In this short term prime only study, a vaccine formulation containing the combined adjuvant system had better protection against plague as compared with those containing individual adjuvants. On the other hand, in the prime-boost long term study, vaccination with $\mathrm{rF} 1-\mathrm{V}$ antigen (different doses) and SA-4-1BBL generated significant titers of anti-rF1-V antibodies that lasted over a 190-day observation period and mice immunized with lowest dose of $\mathrm{rF} 1-\mathrm{V}$ antigen and SA-4-1BBL were completely protected against plague, displaying a dose- 
response. Taken together, our data demonstrate that SA-4-1BBL improves the efficacy of the lead $\mathrm{rF} 1-\mathrm{V}$ vaccine adjuvanted with alum for the generation of $\mathrm{Th}_{1}$ responses and $\mathrm{SA}$ 4-1BBL alone generates a long-lasting anti- $\mathrm{rF1}-\mathrm{V}$ humoral response and protects from plague in a prime-boost setting. This study provides the scientific rationale for the use of SA-4-1BBL for the development of subunit vaccines against infections that may benefit from a balanced $\mathrm{Th}_{1} / \mathrm{Th}_{2}$ response.

\section{Materials and Methods}

\section{Mice, vaccinations, and bacterial challenge}

Female, 6 to 8-week-old C57BL/6 mice were purchased from The Jackson Laboratory or bred at the University of Louisville. Animals were cared for in accordance with NIH guidelines and all procedures were approved by the University of Louisville IACUC (Protocol No. 10-117 and 13-080). In the prime only short term study, vaccine formulations were prepared and incubated at $4{ }^{\circ} \mathrm{C} \mathrm{O} / \mathrm{N}$ to allow for the adsorption of the antigen to alum, and mice were vaccinated once subcutaneously (s.c.) on day 0 with 0.2 $\mathrm{ml}$ of one of the following vaccine formulations containing $1 \mu \mathrm{g}$ of $\mathrm{rF} 1-\mathrm{V}$ protein: 1$) \mathrm{rF} 1-$ $\mathrm{V}, 2) \mathrm{rF} 1-\mathrm{V}+\mathrm{SA}-4-1 \mathrm{BBL}(12.5 \mu \mathrm{g}), 3) \mathrm{rF} 1-\mathrm{V}+$ alum $(200 \mu \mathrm{g})$, or 4) rF1-V + SA-41BBL $(12.5 \mu \mathrm{g})+$ alum $(200 \mu \mathrm{g})$. Anti-rF1-V antibody titers were assessed using serum collected on days 14 and 28 post-vaccination by sub-mandibular bleed.

In the prime-boost long term study, mice were vaccinated twice subcutaneously (s.c.) on days 0 and 21 with $0.2 \mathrm{ml}$ of one of the following vaccine formulations containing rF1-V protein: 1) $5 \mu \mathrm{g} r F 1-\mathrm{V}, 2) 1 \mu \mathrm{g} r F 1-\mathrm{V}+\mathrm{SA}-4-1 \mathrm{BBL}(12.5 \mu \mathrm{g}), 3) 5 \mu \mathrm{g}$ $\mathrm{rF} 1-\mathrm{V}+\mathrm{SA}-4-1 \mathrm{BBL}(12.5 \mu \mathrm{g})$, or 4) $10 \mu \mathrm{g} \mathrm{rF} 1-\mathrm{V}+\mathrm{SA}-4-1 \mathrm{BBL}(12.5 \mu \mathrm{g})$. Anti-rF1-V 
antibody titers were assessed using serum collected on days 14, 28, 35, 58, 98, 147, and 190 post-prime by sub-mandibular bleed.

For bubonic plague infection studies, immunized mice were transferred and maintained in the ABSL-3 vivarium at the University of Louisville's Center for Predictive Medicine Regional Biocontainment Laboratory. In the prime only setting 35 days postvaccination, and in prime-boost setting 202 days post-prime mice were lightly anesthetized with isoflorane and inoculated intradermally (i.d.) at the base of the tail with $20 \mu 1$ of $\sim 200$ colony forming units of fully virulent $Y$. pestis CO92 Lux PcysZK [137]. Mice were monitored twice daily for survival and moribund animals were humanly euthanized. Bacterial dissemination was assessed by optical imaging and images were taken using the IVIS Spectrum In Vivo Imaging System (PerkinElmer, Waltham, MA). Regions of interest within the images were generated using the LivingImage 4.4 (PerkinElmer) to calculate the average radiance (photons $/ \mathrm{sec} / \mathrm{cm}^{2}$ ) at the site of infection $[137,138]$.

\section{Reagents and antibodies}

Y. pestis recombinant $\mathrm{rF} 1-\mathrm{V}$ fusion protein (NR-4526) was obtained through the NIH Biodefense and Emerging Infections Research Resources Repository, NIAID, NIH by BEI Resources. SA-4-1BBL protein was expressed and purified in the laboratory using standard techniques as previously published [101]. Alhydrogel was purchased from Brenntag Biosector, Denmark. Anti-CD8-APC-Cy7, anti-CD4-Alexa700, anti-CD44APC, anti-TNF $\alpha-P E$, anti-IFN $\gamma$-PE-Cy7, and isotype-matched antibodies with the same fluorochromes were purchased from BD Bioscience, eBioscience, or BioLegend, and used for flowcytometry.

\section{Intracellular cytokine analysis}


In the short term study, C57BL/6 mice were vaccinated twice s.c. on day 0 and 35 with vaccine formulations containing $1 \mu \mathrm{g}$ of $\mathrm{rF} 1-\mathrm{V}$ protein: 1$) \mathrm{rF} 1-\mathrm{V}, 2) \mathrm{rF} 1-\mathrm{V}+\mathrm{SA}-4-$ 1BBL (12.5 $\mu \mathrm{g}), 3) \mathrm{rF} 1-\mathrm{V}+$ alum $(200 \mu \mathrm{g})$, or 4) rF1-V + SA-4-1BBL $(12.5 \mu \mathrm{g})+$ alum $(200 \mu \mathrm{g})$. Lymphocytes were isolated from draining lymph nodes five days after booster vaccination (Day 40) and processed into single-cell suspension. For intracellular cytokine analysis, $0.5 \mathrm{ml}$ of lymphocytes $\left(8 \times 10^{6}\right.$ cells $\left./ \mathrm{ml}\right)$ were plated in a 48 well-plate and stimulated with $20 \mu \mathrm{g}$ of $\mathrm{rF} 1-\mathrm{V}$ protein in the presence of $20 \mathrm{U}$ IL-2 and $25 \mathrm{ng}$ SA-41BBL for $24 \mathrm{~h}$. Cultures without $\mathrm{rF} 1-\mathrm{V}$ served as controls. Golgi Plug $(1 \mu \mathrm{l} / \mathrm{mL}$, BD PharMingen) was added to the cultures during the last $4 \mathrm{~h}$ of incubation. Cells were surface stained first with anti-CD4-Alexa700, anti-CD8-APC-Cy7, and anti-CD44-APC and then fixed with $4 \%$ paraformaldehyde for $15 \mathrm{~min}$. Following permeabilization, cells were stained with anti-IFN $\gamma$-PE-Cy7, anti-TNF $\alpha-\mathrm{PE}$, or isotype controls, acquired using multiparameter flow cytometer (BD FACS LSR-II), and analyzed by FACSDiva software.

\section{Anti-rF1-V antibody analysis}

The titers of anti-rF1-V antibodies in vaccinated $\mathrm{C} 57 \mathrm{BL} / 6$ mice were determined using a standard ELISA. Briefly, 96-well titer plates were coated with $1 \mu \mathrm{g} / \mathrm{ml}$ of $\mathrm{rF} 1-\mathrm{V}$ overnight at $4{ }^{\circ} \mathrm{C}$, blocked with PBS containing 5\% dry milk $+0.5 \%$ Tween 20 for 45 minutes at RT, and then washed with PBS $+0.5 \%$ Tween 20 . Fifty $\mu 1$ of two-fold serial dilutions of serum was added to wells and incubated for $90 \mathrm{~min}$ at RT. Wells were then washed 3 times with PBS $+0.5 \%$ Tween 20 and incubated with anti-mouse IgG-, $\operatorname{IgG}_{1^{-}}$ or $\operatorname{IgG}_{2 \mathrm{c}}$-HRP (Sigma and Jackson ImmunoResearch) for $60 \mathrm{~min}$. Wells were washed 3 times with PBS $+0.5 \%$ Tween 20, incubated with fifty ul TMB substrate (BD 
biosciences) for $30 \mathrm{~min}$, and reaction was stopped with $2 \mathrm{~N}$ sulfuric acid. Absorbance was measured at $450 \mathrm{~nm}$ and anti-rF1-V antibodies were reported as $\log _{10}$ titers of the greatest serial dilution with a mean $\mathrm{OD}_{450}>$ two-fold the $\mathrm{OD}_{450}$ value of naïve serum with the same dilution. Samples with an antibody titer of $\log _{10} 1.4$ or less were considered negative.

\section{Statistical analysis}

Analysis of variance (ANOVA) was used to compare antibody titers and $\mathrm{CD} 4^{+}$ and $\mathrm{CD} 8^{+}$effector responses among experimental groups. The Student t-test (2-tail) was used to compare titers of $\operatorname{IgG}$ subclasses $\left(\operatorname{IgG}_{1}\right.$ and $\left.\operatorname{IgG}_{2 c}\right)$ and the ratios in alum and SA4-1BBL+alum treatment groups. Kaplan-Meier log-rank test was used to generate the survival curves.

\section{$\underline{\text { Results }}$}

\section{SA-4-1BBL and alum as adjuvant platform generates a $T h_{1}$ response to the $r F 1-V$ antigen}

The lead $\mathrm{rF} 1-\mathrm{V}$ vaccine includes alum as a strong adjuvant for the generation of a robust $\mathrm{Th}_{2}$ regulated humoral response in mice $[29,139-141]$. However, alum does not generate antigen-specific $\mathrm{Th}_{1}$ responses as assessed by $\mathrm{Th}_{1}$-regulated $\mathrm{IgG}_{2 \mathrm{a}}$ titers and IFN $\gamma$ production $[140,142]$. Because $\mathrm{Th}_{1}$ cellular response plays an important role in protection against plague and SA-4-1BBL has robust efficacy in generating such a response in other vaccine settings $[98,102,104,105]$, we tested SA-4-1BBL as an adjuvant for the generation of a $\mathrm{Th}_{1}$ response against $\mathrm{rF} 1-\mathrm{V}$ antigen. $\mathrm{C} 57 \mathrm{BL} / 6$ were immunized with vaccine formulations containing $1 \mu \mathrm{g} r \mathrm{~F} 1-\mathrm{V}$ antigen alone or antigen 
admixed with $12.5 \mu \mathrm{g}$ of SA-4-1BBL, $200 \mu \mathrm{g}$ alum, or a mixture of both adjuvants followed by a booster injection 35 days later. As expected, immunization with $\mathrm{rF1} 1 \mathrm{~V}$ alone or $\mathrm{rF} 1-\mathrm{V}+$ alum did not result in significant increases in the absolute numbers of $\mathrm{CD}^{+}$or $\mathrm{CD} 8^{+} \mathrm{T}$ cells expressing the $\mathrm{Th}_{1}$ signature cytokine IFN $\gamma$ and proinflammatory cytokine TNF $\alpha$ (Fig. 1). In contrast, we observed elevated numbers of both $\mathrm{CD}^{+}$(Fig. 1A-B) and $\mathrm{CD} 8^{+}$(Fig. 1C-D) $\mathrm{T}$ cells producing TNF $\alpha$ and IFN $\gamma$ from mice immunized with $\mathrm{rF} 1-\mathrm{V}+\mathrm{SA}-4-1 \mathrm{BBL}$. Interestingly, in spite of the $\mathrm{Th}_{2}$ bias of alum, the combined adjuvant platform was even more effective in generating TNF $\alpha$ and IFN $\gamma$ responses, with the only exception being in the IFN $\gamma$ response in $\mathrm{CD}^{+} \mathrm{T}$ cells, where SA-4-1BBL performed as well as the combined adjuvant system (Fig. 1D). In this model, generation of more robust $\mathrm{Th}_{1}$ response with SA-4-1BBL + alum therapy is consistent with previous findings where alum adsorbed IL-12 promoted antibody as well as type 1 cytokine responses to HIV-1 gp120 [143]. Collectively, these data demonstrate that: i) alum alone does not generate a significant $\mathrm{rF} 1-\mathrm{V}$-specific $\mathrm{Th}_{1}$ cellular response, ii) as an adjuvant SA-4-1BBL induces an $\mathrm{rF1-V-specific} \mathrm{Th}_{1}$ cellular response, and iii) alum is unable to inhibit the ability of SA-4-1BBL to generate a $\mathrm{Th}_{1}$ response and in the contrary significantly improves such a response.

\section{SA-4-1BBL and alum as adjuvant platform generates high antibody titers against} rF1-V in a prime-only setting

While SA-4-1BBL has been shown in multiple vaccines to generate robust antigen specific $\mathrm{CD}^{+}$and $\mathrm{CD}^{+}$cellular immune responses (Fig. 2 and $[102,104]$ ), the ability of SA-4-1BBL to also generate humoral responses has not been examined. Furthermore, while alum did not appear to inhibit the SA-4-1BBL cellular response in the 
combined adjuvant platform, the effect of SA-4-1BBL on alum-generated humoral responses has not been investigated. To address the impact of SA-4-1BBL on the generation of anti-rF1-V antibodies, serum samples were collected from vaccinated mice on days 14 and 28 post-prime and assessed for total IgG titers against rF1-V protein (Fig. 2). Vaccination with $1 \mu \mathrm{g} r \mathrm{~F} 1-\mathrm{V}$ alone did not generate detectable titers at day 14 or 28 , with the exception of one mouse with low IgG titers at day 14 that declined by day 28 , and a separate mouse that did not have titers at day 14 but detectable levels (albeit low) at day 28. SA-4-1BBL therapy alone was also ineffective in generating a sustained antibody response and all animals in this group were negative for anti-rF1-V titers by day 28 . In marked contrast, vaccine formulations containing alum rapidly generated significant IgG titers by 14 days post-vaccination (Fig. 2, p < 0.0001) that were maintained for at least 28 days post-vaccination $(\mathrm{p}<0.0001)$. The combined adjuvant platform also yielded a significant increase in antibody titers compared to antigen alone and SA-4-1BBL therapy on days $14(\mathrm{p}<0.0001)$ and $28(\mathrm{p}<0.01)$. However, we did observe slightly lower IgG titers in the combined adjuvant vaccinated mice compared to the alum alone mice at both time points (Alum mean: $14 \mathrm{~d}=3.28 \mathrm{Log}, 28 \mathrm{~d}=3.48 \mathrm{Log}$; Combination: $14 \mathrm{~d}=3.1 \mathrm{Log}$, $28 \mathrm{~d}=3.21 \mathrm{Log} ; \mathrm{p}<0.05$ for both days). Taken together, these data suggest that SA-4$1 \mathrm{BBL}$ in a prime only situation does not elicit a robust $\mathrm{rF} 1-\mathrm{V}$ antibody response. However, SA-4-1BBL addition did not appear to drastically inhibit the generation of antibodies in alum immunized mice.

\section{SA-4-1BBL and alum as adjuvant platform shifts the immune response towards $T_{1}$ direction in a prime-only setting}


Analysis of $\mathrm{T}$ cell responses indicate that SA-4-1BBL generates $\mathrm{Th}_{1}$ cellular responses in vaccinated mice (Fig. 1). Generation of $\mathrm{Th}_{1}$ cytokines by $\mathrm{T}$ cells can also impact the humoral response by altering $\operatorname{IgG}$ class switching from $\mathrm{Th}_{2}$ associated $\operatorname{IgG}_{1}$ to $\operatorname{IgG}_{2 \mathrm{a}}\left(\operatorname{IgG}_{2 \mathrm{c}}\right.$ in $\mathrm{C} 57 \mathrm{BL} / 6$ mice [144]. To determine if SA-4-1BBL influences the generation of IgG subclasses, $\operatorname{IgG}_{1}$ and $\operatorname{IgG}_{2 c}$ serum titers were determined for mice immunized with alum or SA-4-1BBL+alum 28 days post-vaccination. As expected, mice immunized with alum generated a robust $\mathrm{Th}_{2} \mathrm{IgG}_{1}$ antibody response. We also observed a predominantly $\operatorname{IgG}_{1}$ response in animals immunized with the combination adjuvant. However, there was a significant reduction in the $\operatorname{IgG}_{1}$ titers compared to alum only immunized mice (alum mean: 4.34 Log; combination: 3.86 Log; p = 0.0087, Fig.3.A). Moreover, immunization with the combined adjuvant increased the $\mathrm{IgG}_{2 \mathrm{c}}$ response compared to alum. As a result, the combined adjuvant therapy significantly increased the ratio of $\operatorname{IgG}_{2 c}$ to $\operatorname{IgG}_{1}$ compared to the alum alone mice ( $p=0.05$, Fig. 3.B), suggesting a shift in the immune response towards a $\mathrm{Th}_{1}$ direction consistent with the cytokine response (Fig. 3).

\section{Vaccination with combination adjuvant platform provides protection against bubonic plague in a prime-only setting}

Addition of SA-4-1BBL to the rF1-V vaccine significantly enhanced the rF1-Vspecific $\mathrm{Th}_{1}$ immune response in animals. Next we tested whether a vaccine containing SA-4-1BBL could provide protection against pathogen challenge. Four groups of mice $(\mathrm{n}=10)$ were vaccinated once with one of four vaccine formulations: 1) $1 \mu \mathrm{g} \mathrm{rF} 1-\mathrm{V}$ antigen alone, 2) $1 \mu \mathrm{g} r \mathrm{rF} 1-\mathrm{V}+200 \mu \mathrm{g}$ alum, 3) $1 \mu \mathrm{g} \mathrm{rF} 1-\mathrm{V}+12.5 \mu \mathrm{g}$ SA-4-1BBL, or 4) $1 \mu \mathrm{g} r F 1-\mathrm{V}+$ both adjuvants. Serum samples were collected at days 14 and 28 to 
determine anti-rF1-V titers (Fig. 4.A). As seen previously, 30-40\% of mice vaccinated with antigen alone or SA-4-1BBL generated modest titers on days 14 and 28, but significantly higher titers were achieved when alum was present in the vaccine (D14 $p<$ 0.05; D28 $\mathrm{p}<0.01)$. Seven days after the last serum sample, mice were challenged i.d. with fully virulent $Y$. pestis $\operatorname{Lux}_{c y s z}$. Bacterial replication and dissemination were monitored throughout the course of infection by whole animal optical imaging for bacterial bioluminescence. As early as $24 \mathrm{hrs}$ post-infection, we observed a difference in bioluminescence signal at the inoculation site of mice that received alum or SA-4-1BBL+alum compared to those that did not receive alum as part of the vaccine (Fig. 4.C). Furthermore, while the mean bioluminescence of the antigen alone and SA-4-1BBL groups increased at the inoculation site by $48 \mathrm{~h}$ post-infection, indicating bacterial replication, we did not observe an increase in the alum and SA-4-1BBL+alum groups (Fig. 4.D). Eventually, all of the antigen-alone vaccinated animals developed systemic disease (Fig. 4.B) and succumbed to infection by 7.5 days post-infection (Fig. 4.E). We also observed systemic disease in the SA-4-1BBL group. However, $20 \%$ of these mice did not develop systemic disease and eventually cleared the infection (Fig. 4B and E). Interestingly, the two surviving mice did not have detectable antibody titers on day 28 , suggesting that cellular immunity generated by SA-4-1BBL alone was responsible for protection against plague. In contrast to these groups, animals vaccinated with alum demonstrated greater protection, supporting previous studies that anti-rF1-V antibodies generated by alum are important mediators of protection against plague [121, 123, 145]. However, $20 \%$ of the alum group still succumbed to infection (Fig. 4.E). The greatest efficacy was seen in the combination adjuvant platform, in which all mice were protected 
from infection (Fig. 4.E). Taken together, these data demonstrate that: i) while anti-rF1-V antibodies are important mediators of protection, they may not be sufficient to clear infection, as the animals that succumbed to infection in the alum group had similar titers to protected animals, ii) in the absence of protective antibody titers, an anti-rF1-V cellular response can protect against $Y$. pestis infection, as observed in the SA-4-1BBL groups, and iii) generating a balanced $\mathrm{Th}_{1} / \mathrm{Th}_{2}$ immune response by using a combinatorial adjuvant platform improved the outcome of vaccination with either adjuvant individually.

\section{SA-4-1BBL generates a long lasting anti-rF1-V humoral response and protects mice from plague in a prime-boost setting}

Since we observed that, SA-4-1BBL therapy does not elicit a humoral response for $\mathrm{rF} 1-\mathrm{V}$ in a prime-only setting, we wanted to explore the impact of SA-4-1BBL on the generation of anti-rF1-V titers in the prime-boost setting. To test this, mice were vaccinated twice with (1) $5 \mu \mathrm{g} \mathrm{rF} 1-\mathrm{V}$ alone, (2) $1 \mu \mathrm{g} \mathrm{rF} 1-\mathrm{V}+12.5 \mu \mathrm{g}$ SA-4-1BBL, (3) 5 $\mu \mathrm{g} \mathrm{rF} 1-\mathrm{V}+12.5 \mu \mathrm{g}$ SA-4-1BBL, and (4) $10 \mu \mathrm{g} \mathrm{rF} 1-\mathrm{V}+12.5 \mu \mathrm{g}$ SA-4-1BBL with 21 days interval and serum samples were collected from vaccinated mice on days 14, 28, 35, $58,98,147$, and 190 post-prime and assessed for total $\operatorname{IgG}$ titers against $\mathrm{rF} 1-\mathrm{V}$ protein (Fig. 5). Mice that received SA-4-1BBL as part of the vaccine generated significant titers of anti-rF1-V antibodies that lasted over a 190-day observation period. Interestingly, although $5 \mu \mathrm{g} \mathrm{rF} 1-\mathrm{V}$ antigen-alone generated faster and better titers than SA-4-1BBL adjuvanted vaccines early on, these titers were short lived and all mice scored negative by day 98 post-vaccination.

Since we observed that SA-4-1BBL induces the generation of long-lasting humoral response in a prime-boost setting (Fig. 5), and anti-rF1-V antibodies generated 
by alum are important mediators of protection against plague [121, 123, 145], we wanted to examine the protective efficacy of SA-4-1BBL in a long term bubonic model. We challenged the mice i.d. 202 days after the prime with fully virulent $Y$. pestis $\operatorname{Lux}_{c y s Z}$, and monitored the survival and bacterial replication. Eventually, $5 \mu \mathrm{g}$ antigen alone vaccinated mice developed systemic disease (Fig. 6.A) and succumbed to infection by 7 days post-infection (Fig. 6.B). We also observed systemic disease in the $5 \mu \mathrm{g} \mathrm{rF} 1-\mathrm{V}+$ SA-4-1BBL, and $10 \mu \mathrm{g} r F 1-V+$ SA-4-1BBL groups. However, one out of three mice in 5 $\mu \mathrm{g} \mathrm{rF} 1-\mathrm{V}+\mathrm{SA}-4-1 \mathrm{BBL}$ group did not develop systemic disease and eventually cleared the infection (Fig. 6.A-B), while in the $10 \mu \mathrm{g} r \mathrm{~F} 1-\mathrm{V}+\mathrm{SA}-4-1 \mathrm{BBL}$ group all mice developed systemic disease and succumbed to infection by 10.5 days (Fig. 6.A-B). Interestingly, mice vaccinated with $1 \mu \mathrm{g} \mathrm{rF} 1-\mathrm{V}+\mathrm{SA}-4-1 \mathrm{BBL}$ did not develop systemic disease and showed greatest efficacy in which all mice were protected from infection cleared the infection (Fig. 6.A-B). Taken together, these data suggest that SA-4-1BBL in a prime-boost setting induce the generation of long-lasting anti-rF1-V antibody response as compared to antigen alone and provides protection in an antigenic dose-dependent manner.

\section{Discussion}

The lead $\mathrm{rF} 1-\mathrm{V}$ subunit vaccine against plague includes alum as a strong adjuvant for the generation of a robust $\mathrm{Th}_{2}$ regulated humoral response in mice and protects mice against plague $[29,139-141]$. However, the protective efficacy of the vaccine in NHP models has been variable, questioning the benefit of this vaccine for human use [120, 146][147]. This may be due to the inability of the vaccine to generate a $\mathrm{Th}_{1}$ cellular 
response. Indeed, accumulating evidence in the literature suggests that $\mathrm{Th}_{1}$ cellular responses are important and complement humoral responses for protection against plague [129-131]. Alum, as the adjuvant component of the lead $\mathrm{rF1}-\mathrm{V}$ subunit vaccine, does not generate antigen-specific $\mathrm{Th}_{1}$ responses as assessed by $\mathrm{Th}_{1}$-regulated antibody titers and IFN $\gamma$ production $[140,142]$. Therefore, we projected that the $\mathrm{rF1} 1 \mathrm{~V}$ vaccine may benefit from SA-4-1BBL as a strong $\mathrm{Th}_{1}$ adjuvant. Consistent with this notion, a single immunization with SA-4-1BBL + alum adjuvanted $\mathrm{rF} 1-\mathrm{V}$ antigen generated a strong mixed humoral and $\mathrm{Th}_{1}$ cellular immune responses against $\mathrm{rF} 1-\mathrm{V}$ antigen that translated into better protective efficacy against plague in a mouse model as compared with the alum only adjuvanted vaccine. Further, prime-boost immunization with SA-4-1BBL and $1 \mu \mathrm{g} \mathrm{rF} 1-\mathrm{V}$ antigen provided $100 \%$ efficacy in bubonic plague model with generation of a long-lasting antibody response.

Recombinant protein-based subunit vaccines to infectious agents are attractive due to their ease of production and safety features. However, protein-based subunit vaccines heavily depend on immunostimulatory adjuvants for the generation of protective immunity against target infection $[11,12]$. Alum is the most widely used adjuvant and is very effective in generating robust $\mathrm{Th}_{2}$-regulated humoral responses against the antigen component of subunit vaccines [29]. However, cellular immune responses are also important, and in select settings are required for optimal immunity to infections, especially in the case of viruses and intracellular bacterial pathogens. It has been suggested that aluminum adjuvants may not drive complete protection against diseases that require a strong $\mathrm{Th}_{1}$ response, such as malaria and cancer [148]. As such, to improve cellular responses of subunit vaccines, alum based vaccines have been adjuvanted with 
immune modulators, such as IL-12 or synthetic ODNs containing CpG motifs, that promote $\mathrm{Th}_{1}$ cellular responses [149]. We herein proposed that the cellular response and overall efficacy of the plague subunit $\mathrm{rF1}-\mathrm{V}$ vaccine could be improved by using SA-41BBL costimulatory molecule as an adjuvant either alone or in combination with alum. The reasons behind the choice of SA-4-1BBL are several fold. First, signaling via 4-1BB is critical for T cell expansion, survival, acquisition of effector function, and generation of long-term immune memory [90, 96, 97]. Second, a subset of dendritic cells (DCs) constitutively express 4-1BB receptor and signaling via this receptor increases survival of DCs. This leads to increased antigen uptake and cross-presentation to $\mathrm{T}$ cells, and migration to $\mathrm{T}$ cell zone, culminating into enhanced $\mathrm{T}$ cell immune responses $[98,150$, 151]. Third, 4-1BB/4-1BBL interaction selectively promotes the generation/expansion of T cells producing IFN $\gamma$ and TNF $\alpha$ type 1 cytokines [152-154] that play an important role in protection against $Y$. pestis infection [129]. Fourth, 4-1BB expression is inducible and up-regulated on activated $\mathrm{CD}^{+}$and $\mathrm{CD} 8^{+} \mathrm{T}$ cells [70]. This provides an opportunity to specifically target antigen-specific, activated $\mathrm{T}$ cells for a more robust response, and as such avoid systemic/nonspecific activation of immune responses and associated adverse effects reported for other $\mathrm{Th}_{1}$ immunomodulators, such as IL-2, IL-12 or CpG ODN [155-157]. Finally, and important in the context of this study, 4-1BB costimulation primarily and directly works on T cells, whereas alum targets APCs $[29,30]$ and as such these two adjuvants may work in synergy to enhance immunity against $\mathrm{rF} 1-\mathrm{V}$ antigen.

A prime-boost vaccination with $\mathrm{rF1}-\mathrm{V}+\mathrm{SA}-4-1 \mathrm{BBL}$ resulted in increased numbers of both $\mathrm{CD}^{+}$and $\mathrm{CD} 8^{+} \mathrm{T}$ cells producing $\mathrm{TNF} \alpha$ and IFN $\gamma$. In particular, the most significant increase was observed for $\mathrm{CD} 8^{+} \mathrm{T}$ cells producing IFN $\gamma$. Given the 
recently demonstrated role of $\mathrm{CD}^{+} \mathrm{T}$ cells in LcrV DNA-based vaccine protection against $Y$. pestis infection [158], our observations are significant. The administration of SA-4-1BBL with alum further improved the $\mathrm{Th}_{1}$ cellular responses, except for IFN $\gamma$ in $\mathrm{CD}^{+} \mathrm{T}$ cells where SA-4-1BBL therapy performed as well as the combination therapy. These findings are consistent with the previously published studies by us $[102,103]$ and others $[159,160]$ demonstrating the $\mathrm{CD} 8^{+} \mathrm{T}$ cell-centric function of $4-1 \mathrm{BB}$ signaling. Importantly, our findings are consistent with previously published studies demonstrating that alum in combination with $\mathrm{Th}_{1}$ immune modulators, such as IL-12 or TLR4 agonists, promotes both $\mathrm{Th}_{2}$-mediated humoral as well as $\mathrm{Th}_{1}$ cytokine responses $[6,143]$.

We observed that SA-4-1BBL alone increased the number of $\mathrm{CD}^{+} \mathrm{T}$ cells producing $\mathrm{TNF} \alpha$ and IFN $\gamma$ as compared with alum, but such increases were not statistically significant. Alum when used in combination boosted the activity of SA-41BBL, resulting in a statistically significant increase in the number of $\mathrm{CD}^{+} \mathrm{T}$ cells producing TNF $\alpha$ and IFN $\gamma$. These observations are novel as they demonstrate synergy between alum and SA-4-1BBL in generating a strong $\mathrm{CD}^{+} \mathrm{Th}_{1}$ cellular response. It has been shown that $\mathrm{CD}^{+} \mathrm{T}$ cells are required for the protective efficacy against $Y$. pestis conferred by $\mathrm{CD}^{+} \mathrm{T}$ cells in an adoptive transfer model [161]. Therefore, this synergy has important implications for the development of vaccines against intracellular infections where $\mathrm{CD}^{+} \mathrm{Th}_{1}$-regulated $\mathrm{CD} 8^{+}$cytotoxic $\mathrm{T}$ cell responses play important roles in clearance. Further, treatment with $\mathrm{Th}_{1}$ cytokines $\mathrm{TNF} \alpha$ and IFN $\gamma$ has been shown to diminish the survival of $Y$. pestis within the macrophages [162].

However, $\mathrm{CD}^{+} \mathrm{T}$ cells are not only critical for the generation of primary $\mathrm{CD} 8^{+} \mathrm{T}$ cell responses and the establishment of long-term memory, but also play important roles 
in the generation and establishment of B cell responses that produce Ig isotypes, such as $\operatorname{IgG}_{2 \mathrm{a}}\left(\mathrm{IgG}_{2 \mathrm{c}}\right.$ in case of $\left.\mathrm{C} 57 \mathrm{BL} / 6\right)$, involved in opsonization [163]. Although $Y$. pestis can evade from phagocytosis [164], it has been reported that neutrophils can control the bacteria growth within the first 2 days after infection [162]. Moreover, anti-LcrV antibodies enhances the $Y$. pestis phagocytosis indirectly by inhibiting Yop translocation [165] or production within the cells, and neutrophils are the major cell types in this protection against Y.pestis [166]. Here, we observed an increase in $\mathrm{IgG}_{2 \mathrm{c}}$ antibody response while there was a significant decrease in $\operatorname{IgG}_{1}$ antibody response in mice vaccinated with the adjuvant system. As a result, the ratio of $\operatorname{IgG}_{2 c}$ to $\operatorname{IgG}_{1}$ was significantly increased, although the $\operatorname{IgG}_{1}$ was the predominant isotype. Cytokines can regulate the generation of antibody isotypes, for example, IFN $\gamma$ prevents IL-4 induced $\mathrm{IgG}_{1}$ responses, while IL-4 blocks IFN $\gamma$ induced $\operatorname{IgG}_{2 \mathrm{a}}$ response [167]. As a result, the observed increased ratio of $\operatorname{IgG}_{2 c}$ to $\operatorname{IgG}_{1}$ in the combined adjuvant therapy might be due to the elevated levels of IFN $\gamma$ in these animals. Although all $\operatorname{IgG}$ subclasses contribute to clearance of bacteria, $\operatorname{IgG}_{2 a}$ can fix the complement system greater than $\operatorname{IgG}_{1}$ [163]. Therefore it can enhance the phagocytosis of $Y$. pestis by neutrophils [168].

Many studies have shown that anti-rF1-V antibodies contribute significantly to protection and that passive transfer of antibodies can confer protection in mice challenged with $Y$. pestis $[121,123,145]$. Our data do not contradict these assumptions, as in the prime-only study, the alum alone animals had high antibody responses to $\mathrm{rF} 1-\mathrm{V}$ and $80 \%$ of these animals were protected against $Y$. pestis challenge. However, $20 \%$ of these mice still succumbed to infection despite no significant differences in anti-rF1-V titers, suggesting that while important, antibodies alone may not be sufficient for protection. 
This notion is consistent with studies in nonhuman primates demonstrating lack of correlation between antibody titers and protection against plague [169]. Cynomolgus macaques generated high and consistent titers of $\mathrm{F} 1$ and $\mathrm{V}$ antibodies and there was a correlation with protection, while African green monkeys generated more individual titers of $\mathrm{V}$ antibodies and they did not show a correlation [170]. Furthermore, in the prime-only setting, SA-4-1BBL alone provided $20 \%$ protection, in spite of no detectable antibody titers against $\mathrm{rF} 1-\mathrm{V}$ in the mice that cleared infection, and mice immunized with the combined adjuvant were $100 \%$ protected in spite of having lower IgG titers compared to alum alone therapy. These observations are consistent findings from Elvin and Williamson which demonstrated that protection conferred by a similar F1 and V subunit vaccine requires a $\mathrm{Th}_{1}$ response. They reported that mice lacking the signal transducer and activator of transcription protein family 4 (Stat4), which regulates the $\mathrm{Th}_{1}$ response, were not protected against plague following vaccination. [171]. In marked contrast, mice deficient in Stat6, which regulates the $\mathrm{Th}_{2}$ response, had vaccine-induced protection. Importantly the antibody titers and IgG isotypes in both mice were similar, providing direct evidence for an important role of the $\mathrm{Th}_{1}$ cellular response in protection. This notion is further consistent with the studies reporting important roles of $\mathrm{T}$ cells and $\mathrm{Th}_{1}$ cytokines IFN $\gamma$ and $\mathrm{TNF} \alpha$ in protection against $Y$. pestis $[129,131]$.

However, in the case of prime-boost study, SA-4-1BBL adjuvanted vaccine with different $\mathrm{rF} 1-\mathrm{V}$ doses generated significant titers of anti-rF1-V antibodies that lasted over a 190-day observation period. On the other hand, vaccination with $\mathrm{rF} 1-\mathrm{V}$ alone was failed to do so. Although it has been demonstrated that agonistic 4-1BB antibody suppresses the $\mathrm{T}$ cell dependent humoral immunity through the induction of $\mathrm{CD} 4^{+} \mathrm{T}$ helper cell anergy 
[172], and treatment of several autoimmune diseases have taken advantage from this $[173,174]$, it has also been reported that, the form of 4-1BB agonistic adjuvant can also influence the outcome of the cellular and humoral immune responses [175]. As such, treatment with agonistic anti-4-1BB antibody, and secreted form of the 4-1BBL suppress the humoral response to HIV-1 Gag DNA vaccine, while DNA plasmid expressing membrane bound 4-1BBL enhances the Gag-specific antibody titers [175]. Therefore, generation of long-lasting anti-rF1-V titers with the natural soluble form of SA-4-1BBL adjuvanted vaccine may not be surprising, and may need a booster dose as seen in primeboost setting for the generation of potent humoral response since in the prime-only setting we did not observe an increase in the antibody response against $\mathrm{rF} 1-\mathrm{V}$ antigen. Interestingly, here we observed an antigen-dose dependent efficacy of SA-4-1BBL therapy where administration of SA-4-1BBL with $1 \mu \mathrm{g} \mathrm{rF} 1-\mathrm{V}$ resulted in complete protection, while increasing the antigen dose abrogated the protection to $33 \%$ and $0 \%$ with $5 \mu \mathrm{g}$ and $10 \mu \mathrm{g} \mathrm{rF} 1-\mathrm{V}$, respectively. This could be due to the over-activation of $\mathrm{CD} 8^{+} \mathrm{T}$ cells with higher antigen doses, and subsequent generation of $\mathrm{CD} 8^{+} \mathrm{T}$ suppressor cells which inhibit or suppress $\mathrm{CD}^{+} \mathrm{T}$ cell responses via IFN $\gamma$-dependent release of TGF $\beta$ [176]. On the other hand, vaccination with $1 \mu \mathrm{g} r F 1-V$ and SA-4-1BBL induced the generation of a robust long-lasting antibody response with a balanced effector cellular response which resulted in $100 \%$ efficacy in bubonic plague model in the prime-boost study.

Collectively, our data demonstrate that in the short term setting, addition of the $\mathrm{Th}_{1}$ adjuvant SA-4-1BBL improves the efficacy of the lead alum adjuvanted $\mathrm{rF} 1-\mathrm{V}$ subunit vaccine by generating a strong $\mathrm{Th}_{1}$ cellular immune response without significant 
impact on the generation of $\mathrm{Th}_{2}$ regulated antibody responses. The combined adjuvant system has better efficacy over individual adjuvants in protection against plague. Importantly, since we did not observe a correlation between antibody titers and survival in mice immunized with SA-4-1BBL (either alone or in combination with alum), we provide the first evidence that $\mathrm{rF} 1-\mathrm{V}$-specific cellular immune responses can protect against plague and may provide an important contribution to the future success of the $\mathrm{rF} 1-\mathrm{V}$ subunit plague vaccine. This hypothesis is supported by previous reports that show that adaptive cellular modulators, such as $\mathrm{CpG}$ ODN or cytokines, can enhance the immune response in alum vaccinated animals [143, 177]. However, giving one more shot of SA-4-1BBL adjuvanted $\mathrm{rF} 1-\mathrm{V}$ vaccine induces the generation of long-lasting antibody response against the antigen, and confers $100 \%$ protection, highlighting the importance of humoral immune response for $Y$. pestis. Therefore, generation of a balanced $\mathrm{Th}_{1}$ and $\mathrm{Th}_{2}$ immune response is critical for the elimination of bacteria. Finally, while these studies focused on the $\mathrm{rF} 1-\mathrm{V}$ subunit vaccine, we believe that our findings are not specific to $\mathrm{rF} 1-\mathrm{V}$ or plague, and that addition of SA-4-1BBL to other subunit vaccines will have similar effects and could dramatically improve efficacy of vaccines, especially against intracellular pathogens. 
A

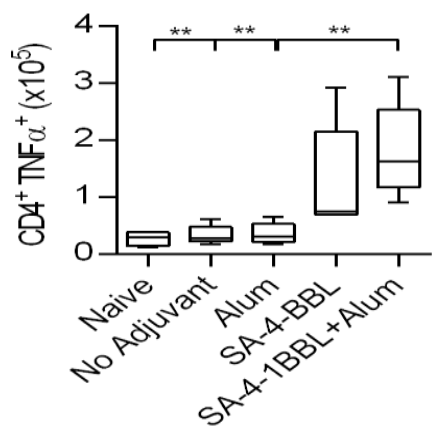

C

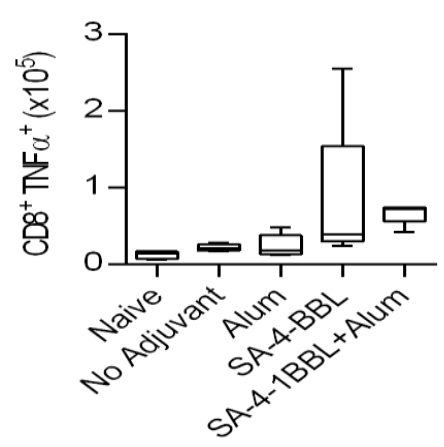

B

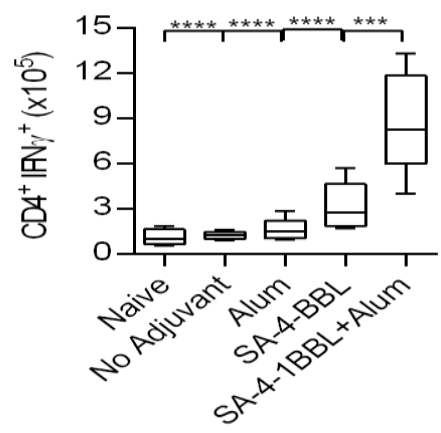

D

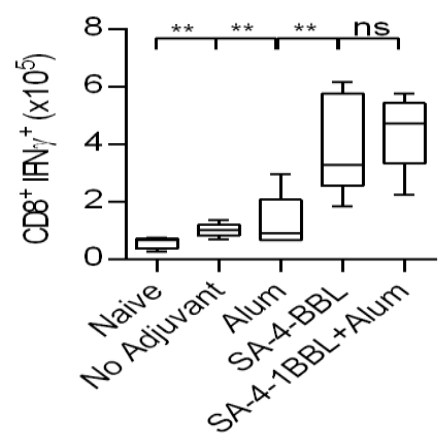

\section{Figure 1}

$\mathrm{SA}-4-1 \mathrm{BBL}+$ Alum increases the cellular response of the plague vaccine. C57BL/6 mice were immunized with $\mathrm{rF} 1-\mathrm{V}$ with or without the indicated adjuvants or PBS only $(\mathrm{n}=5)$ on days 0 and 35.5 days after the boost (Day 40), antigen specific cytokine response was determined in the dLN. Each bar represents the mean and S.D. of absolute numbers of $\mathrm{CD} 4{ }^{+} \mathrm{CD} 44^{\text {hi }}$ and $\mathrm{CD} 8{ }^{+} \mathrm{CD} 44^{\text {hi }} \mathrm{T}$ cells producing TNF $\alpha$ (left) and IFNy (right) cytokines in each group. Data are representative of two independent experiments. Upper panel shows the absolute numbers of $\mathrm{CD}^{+} \mathrm{T}$ cells, lower panel shows the absolute numbers of $\mathrm{CD}^{+} \mathrm{T}$ cells. ${ }^{* * * *}=\mathrm{p}<0.0001 ; * * *=\mathrm{p}<0.001 ; * *=\mathrm{p}<0.01 ; \mathrm{ns}=$ not statistically significant. 


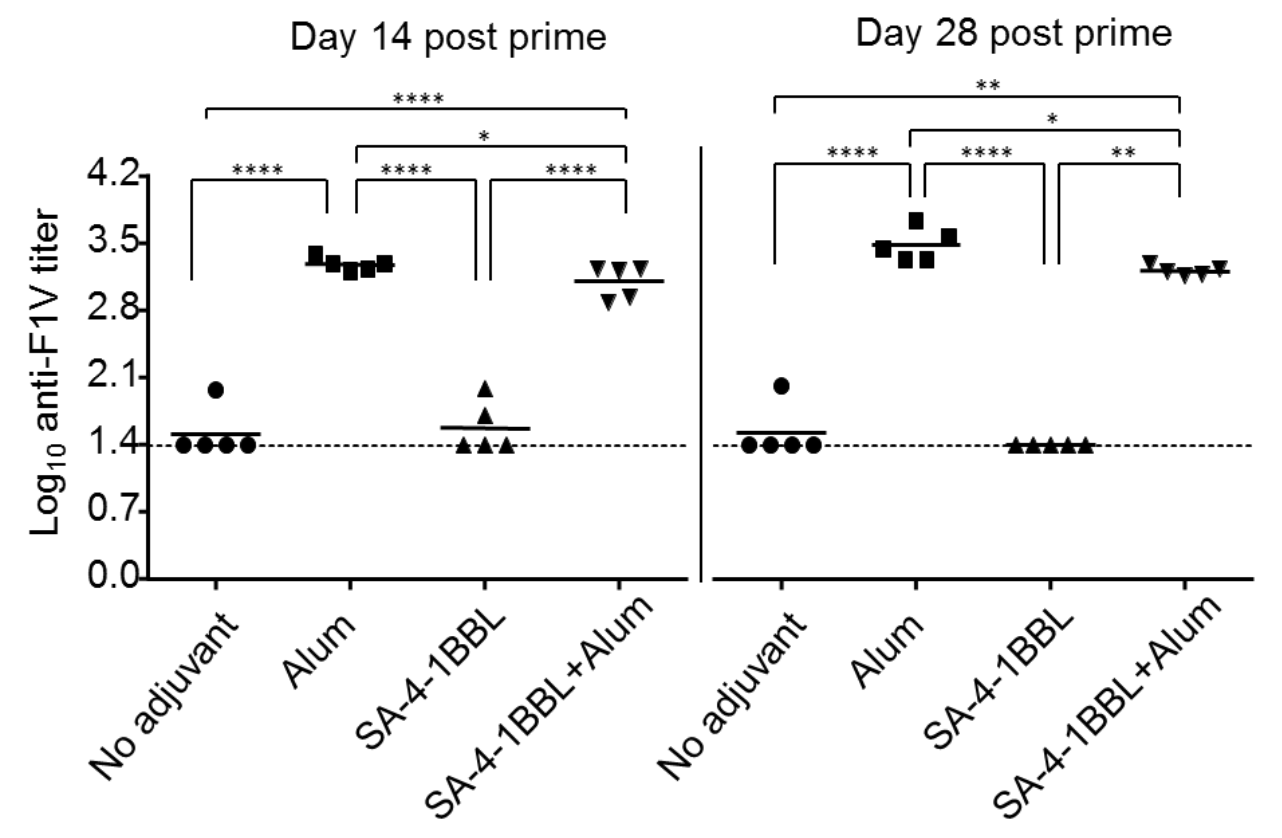

\section{Figure 2}

SA-4-1BBL + Alum induces antibody response for $\mathrm{rF} 1-\mathrm{V}$ in prime-only setting. C57BL/6 mice were immunized with $\mathrm{rF1-V}$ with or without the indicated adjuvants $(n=5) .14$ and 28 days post-immunization serum samples were harvested and IgG titers were determined. Dotted lines indicate limit of detection. Graph shows the individual values in each group. $* * * *=\mathrm{p}<0.0001 ; * *=\mathrm{p}<0.01 ; *=\mathrm{p}<0.05$. 

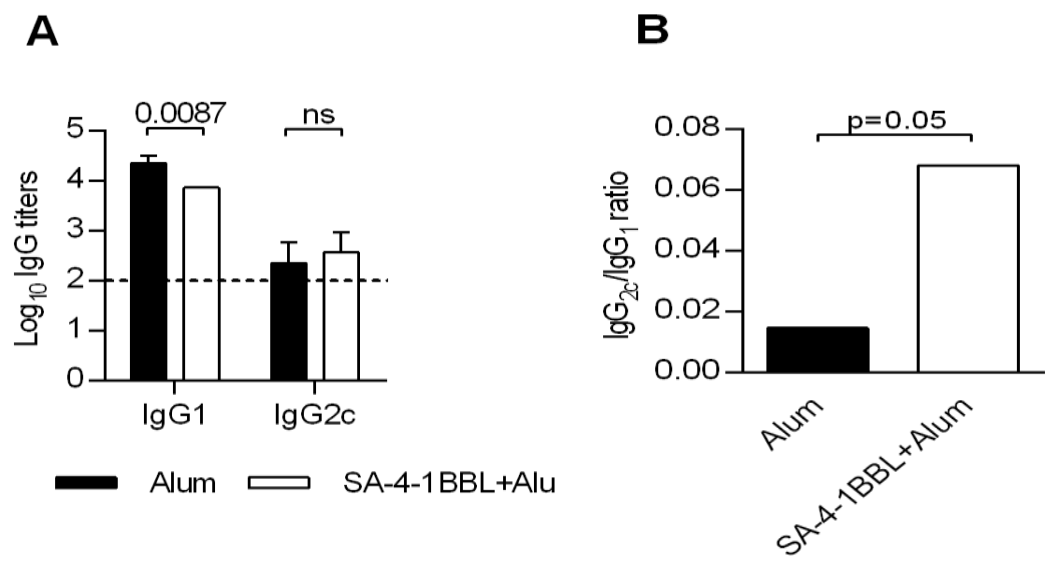

\section{Figure 3}

SA-4-1BBL + Alum shifts the cellular response towards $\mathrm{Th}_{1}$ in prime-only setting. C57BL/6 mice were immunized with $\mathrm{rF1-V}$ and indicated adjuvants $(\mathrm{n}=5) .28$ days postimmunization serum samples were harvested and titers of $\operatorname{IgG}_{1}$ and $\operatorname{IgG}_{2 \mathrm{c}}$ subtypes were determined. A) The bar graph represents average log titers of $\operatorname{IgG}_{1}$ and $\operatorname{IgG}_{2 \mathrm{c}}$ antibodies with SD, and dotted lines indicate limit of detection. Alum (Black); Alum + SA-4-1BBL (White). B) The bar graph represents average $\mathrm{x}$ value of $\mathrm{IgG}_{2 \mathrm{c}} / \mathrm{IgG}_{1}$ ratio in each group. 
A

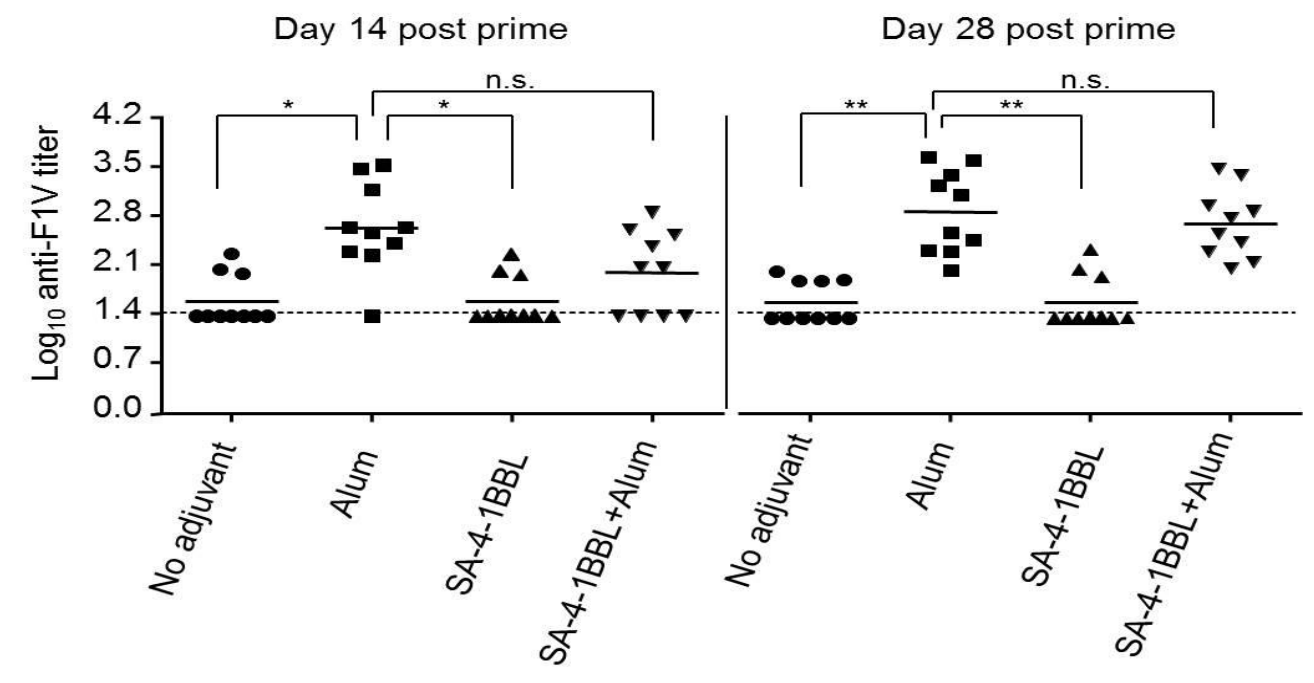

B

C
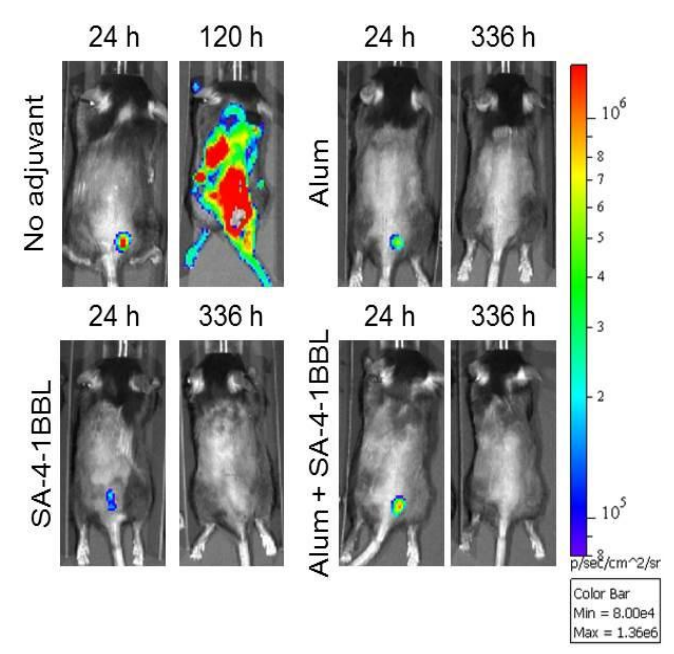

$24 \mathrm{~h}$

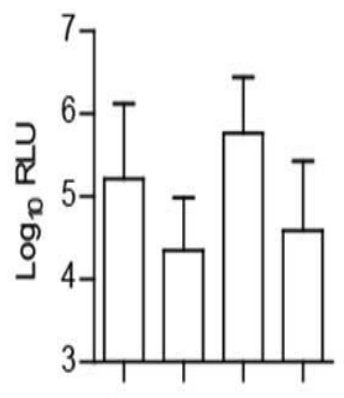

D
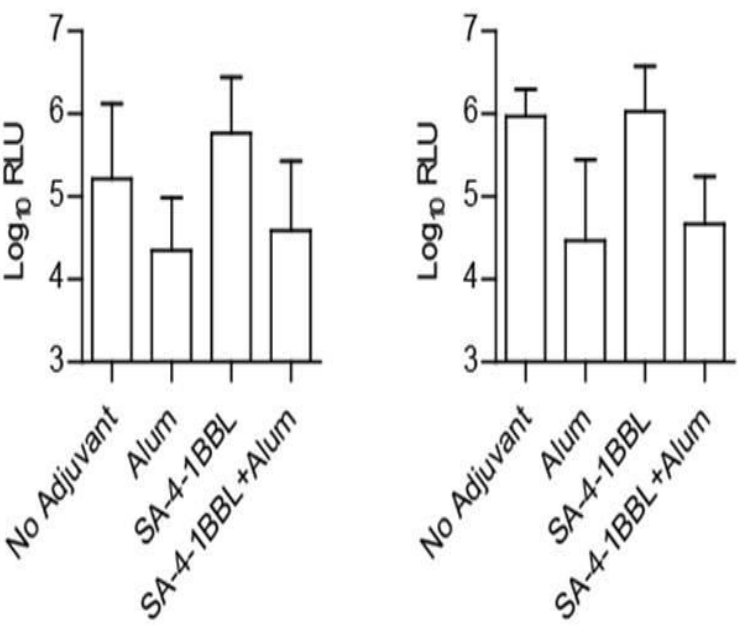


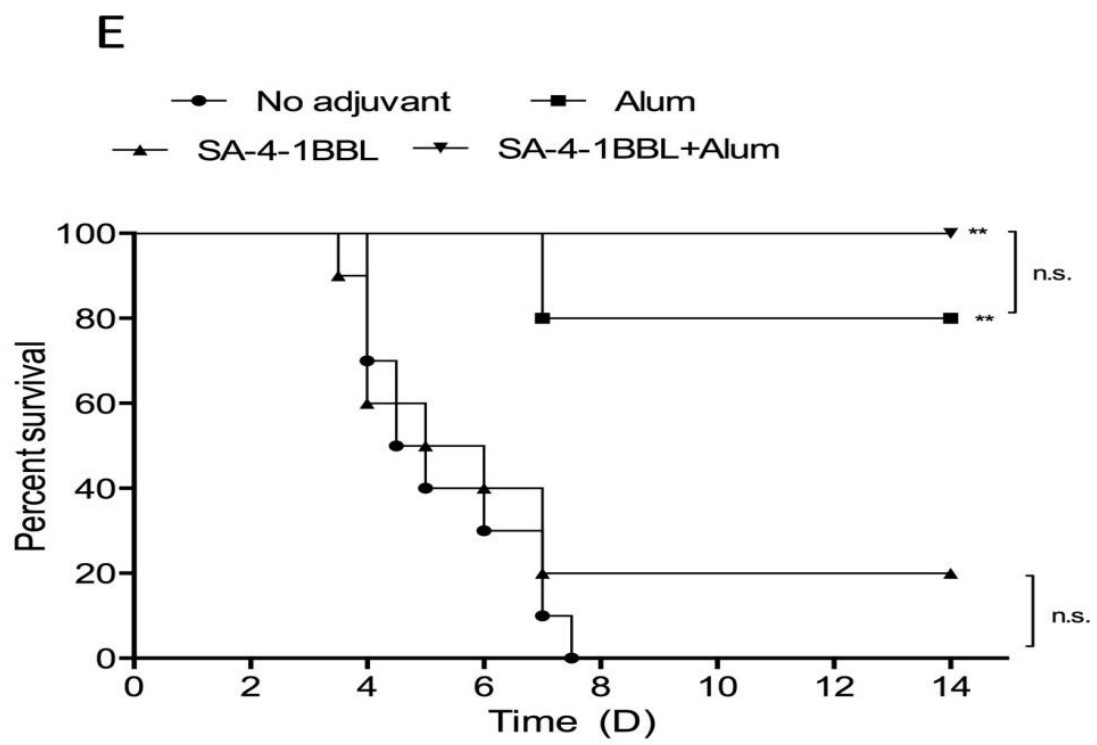

\section{Figure 4}

One shot immunization with SA-4-1BBL + Alum protects mice from bubonic plague. C57BL/6 mice were immunized with $\mathrm{rF1}-\mathrm{V}$ with or without the indicated adjuvants ( $\mathrm{n}=10$ per group). A) $14 \mathrm{~d}$ and $28 \mathrm{~d}$ post-immunization serum samples were harvested and IgG titers determined. Dotted lines indicate limit of detection. 35 days postimmunization mice were challenged with $Y$. pestis Lux $x_{P c y s z K}$ by i.d. injection. B) Representative optical image of animals from each group at $24 \mathrm{~h}$ post-infection or at the time of euthanasia. C) $24 \mathrm{~h}$ and D) $48 \mathrm{~h}$ post-infection bacterial numbers (as a function of bacterial bioluminescence) were determined at the inoculation site by optical imaging (mean radiance (photons $\left./ \mathrm{sec} / \mathrm{cm}^{2}\right) \pm$ st. dev.). E) Survival comparison of vaccinated groups. $^{*}=\mathrm{p}<0.05 ; * *=\mathrm{p}<0.01 ; \mathrm{ns}=$ not statistically significant. 


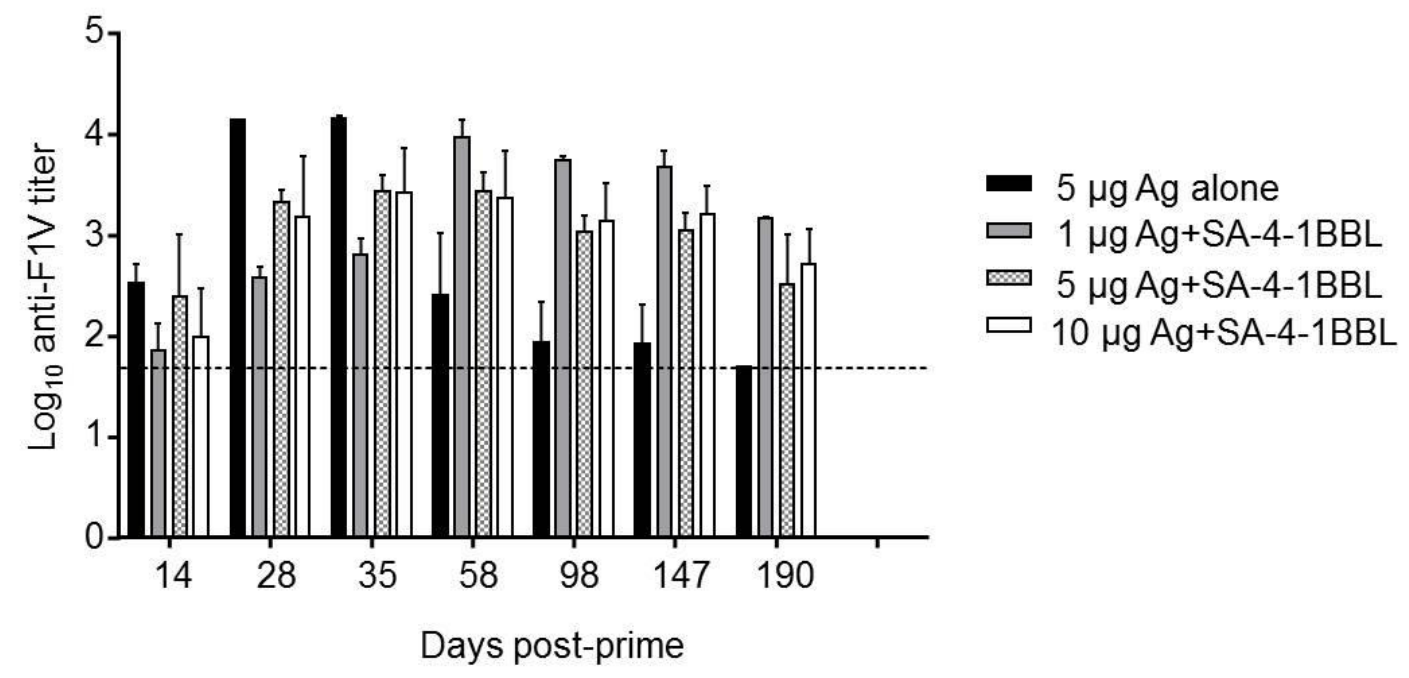

Figure 5

SA-4-1BBL generates a long-lasting anti-rF1-V humoral response in a prime-boost setting. C57BL/6 mice were immunized with vaccine formulations containing (1) $5 \mu \mathrm{g}$ rF1-V alone (n=3), (2) $1 \mu \mathrm{g} r F 1-\mathrm{V}+12.5 \mu \mathrm{g} \mathrm{SA-4-1BBL} \mathrm{(n=3),} \mathrm{(3)} 5 \mu \mathrm{g} \mathrm{rF1-V}+12.5$ $\mu \mathrm{g}$ SA-4-1BBL $(\mathrm{n}=3)$, and (4) $10 \mu \mathrm{g} \mathrm{rF1-V}+12.5 \mu \mathrm{g} \mathrm{SA}-4-1 \mathrm{BBL}(\mathrm{n}=3)$ on days 0 and 21. The role of SA-4-1BBL on humoral immune response was evaluated by the generation of anti-F1-V titers detected in the serum collected on days $14,28,35,58,98$, 147, and 190 post-prime. The bar graph represents average log value of IgG titers in each group. Dotted lines indicate limit of detection. 
A
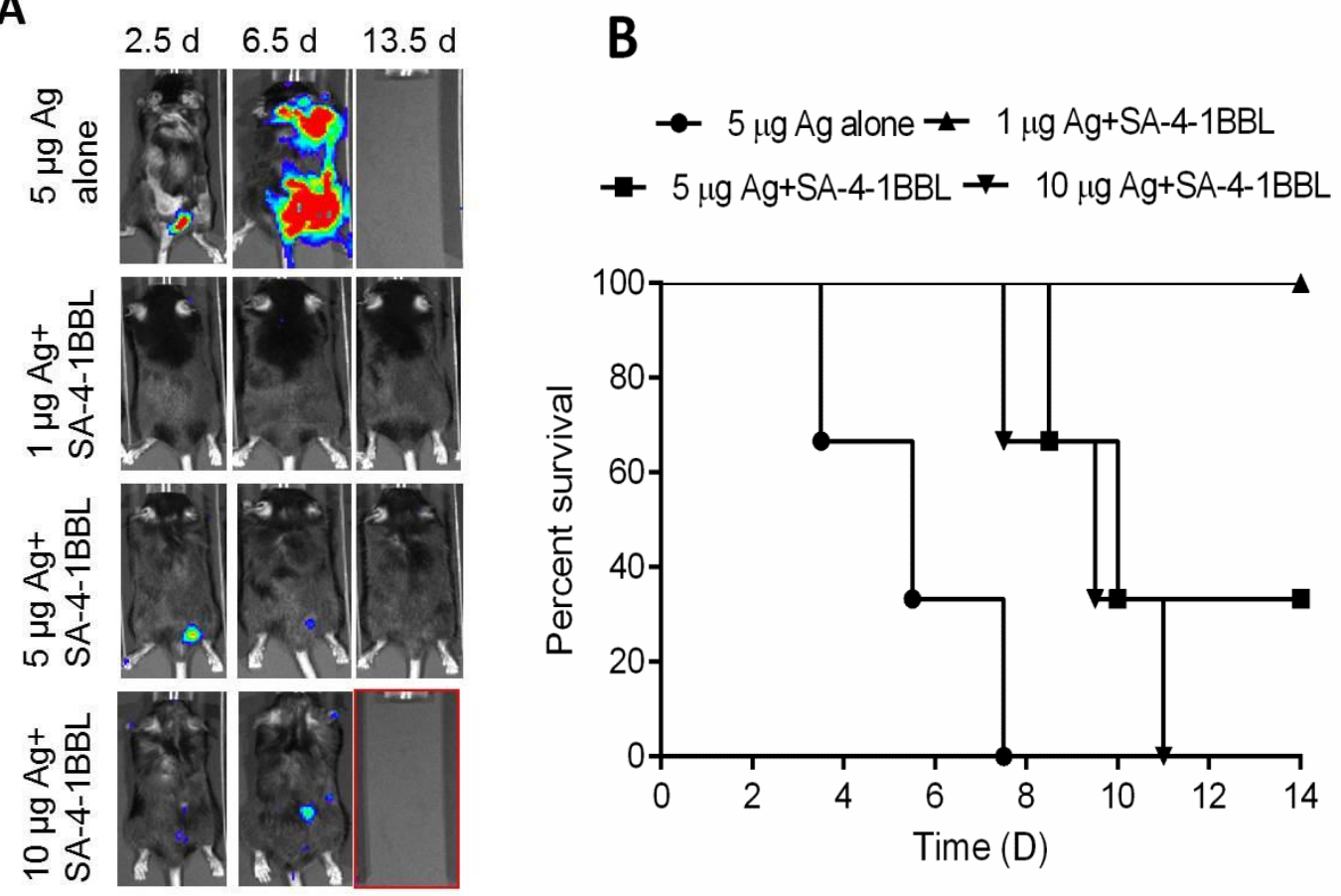

\section{Figure 6}

SA-4-1BBL provides protection against bubonic plague with an $\mathrm{rF1}-\mathrm{V}$ dose dependent manner. C57BL/6 mice ( $\mathrm{n}=3$ per group) were immunized with indicated vaccine formulations on days 0 and 21, and inoculated i.d. at the base of the tail with $\sim 200 \mathrm{CFU}$ of fully virulent $Y$. pestis $\mathrm{CO} 92 \mathrm{Lux}_{\mathrm{Pcys} Z \mathrm{~K}}$. A) Representative optical image of animals from each group at 2.5, 6.5, $13.5 \mathrm{~d}$ post-infection. B) Survival comparison of vaccinated groups. 


\section{CHAPTER 3}

\section{SA-4-1BBL AND MPL COMBINATION AS AN ADJUVANT SYSTEM FOR THE TREATMENT OF BREAST CANCER}

\section{$\underline{\text { Introduction }}$}

Breast cancer is the most common cause of cancer related death among women worldwide. Nearly $30 \%$ of human breast cancers over-express the human epidermal growth factor receptor 2 (Her-2/neu) [178]. Although Her-2/neu positive breast cancer could be treated with trastuzumab, a humanized anti-Her-2/neu mAb, these therapies require frequent administrations and more importantly tumor cells acquire resistance against antibody therapies $[179,180]$. As a result, tumors are being relapsed. Moreover, response rate to trastuzumab therapy is limited in patients with metastatic breast tumor [181]. Therefore, active immunization against Her-2/neu is an alternative approach to $\mathrm{mAb}$ therapy primarily because of the activation of cytotoxic $\mathrm{T}$ cell responses, generation of humoral response and long-term immunological memory to control recurrences [182].

Her-2/neu is an attractive TAA for therapeutic vaccines against Her-2/neu positive breast cancers since it is over-expressed in cancer cells. Active immunization strategies against Her-2/neu include peptide and protein based, DNA based, dendritic cell based, and whole tumor cell based vaccines. In the clinical trials, the mostly tested vaccine strategy is peptide based, since they can induce antigen specific T cell $\left(\mathrm{CD} 8^{+}\right.$and 
$\mathrm{CD}^{+} \mathrm{T}$ cell epitopes) and antibody responses (B cell epitopes). However, this vaccine approach has some limitations due to the restricted immune response to one or two epitopes, or specific HLA haplotypes [182], and without generation of $\mathrm{CD}^{+} \mathrm{Th}$ cells, the induced CTL response cannot be long lived [183]. However, employing protein (entire or truncated) based vaccines may overcome these restrictions since they include both HLA class I and II epitopes, therefore activates both CTL and Th cellular, and humoral responses. As such, in several clinical trials it has been reported that use of Her-2/neu protein as a vaccine antigen, induces antigen specific $\mathrm{T}$ cell and antibody responses [184][185].

Even though Her-2/neu is an immunogenic molecule since it generates specific antibody and CTL responses in some patients [16, 17], soluble Her-2/neu protein as a recombinant vaccine is not immunogenic [186]. Therefore, to generate potent immune responses to Her-2/neu protein, adjuvants should be formulated in Her-2/neu recombinant vaccines [184]. In cancer vaccines, induction of $\mathrm{CD}^{+} \mathrm{T}$ cell response is critical to generate potent anti-tumor immune response since antigen specific CTL response is required for the elimination of tumor cells and establishment of long term memory. For antigen specific $\mathrm{T}$ cell activation, the first target among the immune cells is the APCs since in turn they prime the adaptive arm of the immunity. As such, the importance of innate immunity for activation of cellular responses has put the emphasis on the development of adjuvants, which are capable of activating innate immune cells, and the only adjuvants approved by FDA for cancer vaccines are primarily targeting the innate arm of the immune system through APCs. For example, live-attenuated tuberculosis vaccine Bacille Calmette-Guérin (BCG) is the first adjuvant approved by FDA in 1990 
for the treatment of bladder cancer, and MPL is the second one as a component of the adjuvant system (AS04) in Cervarix HPV vaccine [187, 188]. Consistent with the importance of activation of APCs and enhancement of antigen cross-presentation by APCs, vaccination with live attenuated Listeria monocytogenes vector expressing rat or human Her-2/neu gene segments induces tumor regression and prevent metastasis in murine models $[189,190]$. The anti-tumor efficacy of the immunizations against Her2/neu has also been enhanced by targeted delivery of Her-2/neu to the APCs by fusion proteins consisting soluble CTLA-4 [191], or single-chain fragment variable specific for CD11c [192], or Fc domain of IgG [193] in preclinical studies.

Nevertheless, activation of the $\mathrm{CD}^{+}$and $\mathrm{CD}^{+} \mathrm{T}$ cells requires the first signal delivered upon MHC/peptide complex and TCR interaction together with a second signal from costimulatory receptors [8]. Failure to receive second signal can lead to $\mathrm{T}$ cell anergy or apoptosis. Moreover, regulation of Treg suppressive function is another important aim for cancer immunotherapy since tumor-derived soluble factors may induce the generation of regulatory cells as an immune escape mechanism. Among the $\mathrm{T}$ cell costimulatory receptor families, TNFRF signaling is crucial for T cell survival, cell cycle progression, effector function, and memory responses $[69,70]$, and plays important role regulating both adaptive and regulatory immunity. Moreover, these receptors are inducibly expressed on activated $\mathrm{T}$ cells upon antigen stimulation which provides another advantage to serve as adjuvant candidates for vaccines to generate antigen specific immune responses [70].

Since the importance of $\mathrm{CD}^{+} \mathrm{T}$ cells in breast cancer has been determined in preclinical and clinical studies [194-197], we, herein, particularly focused on 4-1BBL 
among TNFRF members due to its critical role in the generation and maintenance of $\mathrm{CD} 8^{+} \mathrm{T}$ cell responses $[92,96]$. Further, 4-1BBL co-stimulation confers $\mathrm{CD} 8^{+} \mathrm{T}$ cells resistant to suppression by Tregs [70], which may help to improve anti-tumor immune responses. However, 4-1BBL functions as a cell membrane protein and has minimal to no function in soluble form. Therefore, we generated a chimeric molecule (SA-4-1BBL) that exists as functional oligomers [102] and showed that SA-4-1BBL induces $\mathrm{T}$ cell activation, acquisition of effector functions, and generation of long-term memory [98, 102, 103, 107, 198]. Most importantly, we reported that SA-4-1BBL modulates regulatory immunity by rendering Teff cells resistant to Treg suppressive activity [98], and inhibiting the conversion of Teff cells into Tregs through the production of IFN- $\gamma$ [106]. As a result, SA-4-1BBL provides significant therapeutic efficacy in various preclinical models [98, 102-104, 136].

Here, we tested the efficacy of a combined adjuvant therapy; SA-4-1BBL, for the activation of adaptive immunity, and MPL, for priming innate immunity, in a preclinical Her-2/neu positive breast cancer model. Since MPL primarily targets APCs, such as DCs and macrophages, for the initiation of adaptive immunity [6] and 4-1BBL targets $\mathrm{CD} 8^{+} \mathrm{T}$ cells for activation, acquisition of effector function, survival, and long-term memory [90, $199,200]$, here we hypothesized that these immunomodulators may work in synergy to improve the anti-tumor efficacy of rat Her-2/neu subunit breast cancer vaccine. We demonstrated that a prime-boost vaccination with combined adjuvant system (SA-4$1 \mathrm{BBL}+\mathrm{MPL}$ ) generates $30 \%$ therapeutic efficacy in Her-2/neu positive A2L2 breast cancer model in BALB/c mice. On the other hand, SA-4-1BBL monotherapy provides $10 \%$ survival, while MPL monotherapy cannot confer therapy. Furthermore, vaccination 
with combined adjuvant system eradicates $20 \%$ of transplanted A2L2 tumors, while depletion of Treg cells further increases its therapeutic efficacy to $40 \%$ in tolerant Her2/neu transgenic BALB/neuT mice. Consistent with the therapeutic efficacy, combined adjuvant therapy induced better tumor specific killing responses than individual agents. Further, combined adjuvant therapy induced significant increase in pro-inflammatory cytokine IFN- $\gamma$ production. Taken together, these data demonstrate the utility of SA-4$1 \mathrm{BBL}+\mathrm{MPL}$ as a novel adjuvant system for the development of therapeutic rat Her2/neu-based breast cancer vaccine with significant therapeutic efficacy.

\section{Materials and Methods}

\section{Cloning, expression, and purification of recombinant rat Her-2/neu ECD}

Extracellular domain (ECD) of the rat Her-2/neu cDNA (from Genscript) was subcloned into both $6 \mathrm{X}$-His-pTWIN-1 and pTWIN-1-6X-His bacterial expression vectors (New England Biolabs) using Nde I and BamH I restriction sites. After transformation into $\mathrm{C} 2566 \mathrm{H}$ competent E. coli cells (New England Biolabs), cultures were grown at $37^{\circ} \mathrm{C}$ for $2 \mathrm{~h}$ and induced using $1 \mu \mathrm{l} / \mathrm{ml}$ of IPTG. After $3 \mathrm{~h}$ induction, cells were harvested, centrifuged, and resuspended in $100 \mathrm{ml}$ lysis buffer containing $20 \mathrm{mM}$ Tris, $\mathrm{pH}$ 7.0, $500 \mathrm{mM} \mathrm{NaCl}, 10 \mu \mathrm{M} \mathrm{ZnCl2,} \mathrm{5mM} \mathrm{imidazole,} \mathrm{and} \mathrm{5mM} \beta$-ME. Cells were lysed using ultra-sonication, inclusion bodies were pelleted at $10,000 \mathrm{xg}$ for $10 \mathrm{~min}$. Inclusion bodies were washed three times by lysis buffer $+1 \%$ Triton X-100 with rotation at RT for $30 \mathrm{~min}$, and centrifuged at $35,000 \mathrm{xg}$ for $30 \mathrm{~min}$. After three cycles, the final pellet was resuspended in $100 \mathrm{ml}$ lysis buffer $+6 \mathrm{M}$ Guanidine $-\mathrm{HCl}$ and rotated $\mathrm{O} / \mathrm{N}$ at $\mathrm{RT}$ to solubilize inclusion bodies. Next day, supernatant was collected after centrifugation at 
$35,000 \times \mathrm{xg}$ for $30 \mathrm{~min}$ at $4^{\circ} \mathrm{C}$, and used for IMAC purification using Talon resin with the advantage of His-Tag according to manufacturer's protocol (Clontech). The only exception was adding $0.1 \%$ Triton $\mathrm{X}-114$ to help to remove endotoxin. In all purification steps, $10 \mu \mathrm{M} \mathrm{ZnCl} 2$ and $5 \mathrm{mM} \beta$-ME were used to enhance proper folding. Protein was dialyzed against PBS, concentrated using an Amnicon Ultra MWCO 50,000, and sterile filtered using a $0.22 \mathrm{~mm}$ filter. Protein concentration was measured using BCA method (Pierce).

ECD of the rat Her-2/neu cDNA (from Genscript) was subcloned into the FlagpMT/BiP vector and expressed in Drosophila Schneider (S2) cells (Drosophila expression system inducible/secreted kit from invitrogen life technologies). Briefly, $9 \times 10^{5}$ cells in 2 $\mathrm{ml}$ serum free media (SFM, Hyclone SFX-insect) containing P/S and L-glu were plated. Cells were led to attach for 1 hour. Meanwhile, transfection solution was prepared by adding solution A consisting $2 \mu \mathrm{g}$ rat Her-2/neu construct and $100 \mathrm{ng}$ of pCoHygro in $100 \mu 1$ SFM to solution B consisting $5 \mu 1$ of Cellfectin in $95 \mu 1$ of SFM and incubated for 45 minutes. After incubation, $0.8 \mathrm{ml}$ SFM was added to Mix A+B to make transfection solution. Growth medium was removed from the cell culture and washed with SFM. $1 \mathrm{ml}$ transfection solution was added to cells and incubated at $27^{\circ} \mathrm{C}$ for $5 \mathrm{~h}$. After 5 hours, transfection solution was removed and replaced with $2 \mathrm{ml} \mathrm{SFM} \mathrm{containing} \mathrm{hygromycin}$ and incubated at $27^{\circ} \mathrm{C}$ for $48 \mathrm{~h}$. Expression of rat Her-2/neu was induced with $1 \mathrm{mM}$ CuSO4 in HyClone (w/o FBS) with the advantage of MT promoter. pMT/BiP vector carrying the Drosophila BiP signal sequence induced rat Her-2/neu protein secretion to the culture supernatant. Culture supernatant was collected and rat Her-2/neu protein was purified and eluted using anti-FLAG M2 affinity gel and 3xFLAG peptide, respectively. 
Protein was dialyzed against PBS, concentrated using an Amnicon Ultra MWCO 50,000, and sterile filtered using a $0.22 \mathrm{~mm}$ filter. Protein concentration was measured using BCA method (Pierce). Construction, expression, purification, and characterization of SA4-1BBL have been previously described [98].

\section{Mice and cell lines}

Female BALB/c and BALBneuT mice were bred in University of Louisville animal facility. All animals were cared for in accordance with institutional and NIH guidelines. Female BALB-neuT mice 6-8 weeks old were used before autochthonous tumor appearance. Heterozygous females expressing rNEU verified by PCR were used throughout this work. Mouse mammary breast cancer cell line A2L2 transfected with rat Her-2/neu $\left(H-2^{\mathrm{d}}\right)$ and its parental 66.3 cell line were kindly provided from Dr. Janet Price from UT M.D. Anderson Cancer Center, and maintained in MEM media supplemented with 5\% FBS, 2X MEM vitamins, $0.1 \mathrm{mM}$ non-essential aminoacids, $1 \mathrm{mM}$ sodium pyruvate, $20 \mathrm{mM}$ HEPES, $2 \mathrm{mM}$ L-glutamine, $1 \% \mathrm{P} / \mathrm{S}$ and $400 \mu \mathrm{g} / \mathrm{ml}$ gentamicin. All the reagents were purchased from Gibco except for FBS from Atlanta biological.

\section{Antibodies and other reagents}

For intracellular cytokine analysis, fluorochrome-conjugated anti-CD3-V500, anti-CD8-APC-Cy7, anti-CD4-Alexa700, anti-CD62L-PerCp-Cy5.5, anti-CD44-APC, anti-CD69-PE, anti-CD25-PE-Cy7, anti-Foxp3-Alexa-488, anti-TNF $\alpha$-PE, anti-IFN $\gamma$-PECy7, anti-IL-2-PerCp-Cy5.5, and isotype matched antibodies were purchased from BD Bioscience, eBioscience, and BioLegend.

For toxicity studies, anti-CD3-FITC, anti- CD8-APC-Cy7, anti- CD4-Alex700, anti- CD11b-PerCp-Cy5.5, anti- CD19-PE-Cy7, anti- NK1.1-PE, anti- F4/80-Alexa647, 
anti- CD11c-PE-Texas Red, and isotype matched antibodies with same fluorochrome were purchased from BD Bioscience, Invitrogen, eBioscience, and Caltag Lab. Fc Block was purchased from BD Bioscience.

MPL was purchased from Sigma Aldrich. Rat Her-2/neu p66 (TYVPANASL) peptide (Her-2 $66-74$ ) corresponding to $\mathrm{H}-2 \mathrm{~K}^{\mathrm{d}}$-restricted, dominant CTL epitope was used for in vitro stimulation of draining lymphocytes in intracellular cytokine assay was purchased from Peptide 2.0 Company.

\section{Tumor models and vaccination}

Tolerant BALB-neuT and BALB/c mice were challenged subcutaneously (s.c.) with $1 \times 10^{5}$ live A2L2 cells in the right flank. For therapy, mice were vaccinated s.c. on days 5 and 10 post-tumor challenge with various vaccine formulations containing rat Her2/neu protein $(50 \mu \mathrm{g})$ with SA-4-1BBL $(25 \mu \mathrm{g})$, or MPL $(25 \mu \mathrm{g})$, or the combination of both agents (25 $\mu \mathrm{g} / \mathrm{agent})$. The doses of SA-4-1BBL, and MPL used in this study were based on our previously published and preliminary studies [98]. Tumor size was measured with a caliper twice weekly. Mice were sacrificed when tumor size reached 12 $\mathrm{mm}$ in diameter, or showed external ulceration, or signs of poor conditions (weight loss, dehydration, inactivity, or moribund). Anti-Her-2/neu antibody titers were assessed using serum collected on termination days when tumor size $12 \mathrm{~mm}$ diameter from tumor bearing mice and on Day 59 from tumor-free (TF) mice. Treg cells were depleted using anti-CD25 Ab (clone PC-61) at $300 \mu \mathrm{g} /$ mice via intra peritoneal (i.p.) injection two days before A2L2 tumor inoculation. In the case of established tumor study, to determine T cell numbers and cytokine responses, mice with 3-5 $\mathrm{mm}$ established tumors were 
vaccinated with indicated formulations and a booster dose was given 5 days later. 3 days after the boost mice were terminated to collect the dLNs.

For toxicity studies, C57BL/6 mice were injected with vaccine formulations containing E7 protein $(50 \mu \mathrm{g})$ with SA-4-1BBL $(25 \mu \mathrm{g})$, or MPL $(25 \mu \mathrm{g})$, or the combination of both agents $(25 \mu \mathrm{g} /$ agent $) .18 \mathrm{~h}$ after immunization, mice were terminated.

\section{Cytotoxicity assay}

$\mathrm{BALB} / \mathrm{c}$ mice were vaccinated twice on days 0 and 5 s.c. with different vaccine formulations containing rat Her-2/neu protein $(50 \mu \mathrm{g})$ with SA-4-1BBL $(25 \mu \mathrm{g})$, or MPL (25 $\mu \mathrm{g})$, or the combination of both agents $(25 \mu \mathrm{g} /$ agent $)$. 5 days after booster vaccination, animals were sacrificed, spleens were harvested and single cell suspensions were prepared. Splenocytes $\left(3 \times 10^{6} / \mathrm{ml}\right)$ from vaccinated animals were re-stimulated in vitro for 5 days with $10 \mu \mathrm{g}$ of Her-2/neu protein/ml in complete MLR medium supplemented with $50 \mathrm{IU} / \mathrm{mL}$ of IL-2 and $0.05 \mu \mathrm{g} / \mathrm{mL}$ of SA-4-1BBL. Viable lymphocytes were harvested 5 days later using a Ficoll gradient and cultured with A2L2, or 66.3 neo, or TC-1 target cells at various ratios for 5 hours at $37^{\circ} \mathrm{C}$. The percentage of killing was determined by the formula: percent specific lysis $=100 \mathrm{x}$ (experimental release - spontaneous release)/ (maximum release - spontaneous release).

\section{Flow cytometry and cytokine bead array analysis}

5 days after booster vaccination, animals were sacrificed, dLNs were harvested, and single cell suspensions were prepared. Lymphocytes $\left(8 \times 10^{6} \mathrm{cell} / \mathrm{s} / \mathrm{ml}\right)$ were plated to the wells of 48 well plate and stimulated with $10 \mu \mathrm{g} / \mathrm{ml} \mathrm{Her-2}$ p 66 peptide for a total of 6 hours and GolgiPlug $(1 \mu \mathrm{l} / \mathrm{ml}$, BD PharMingen) was added at the last 4 hours. Cells were 
surface stained with anti-CD3-V500, anti-CD44-APC, anti-CD8-APC-Cy7 and antiCD4-Alex700, fixed with $4 \%$ paraformaldehyde, and after permeabilization stained intracellular with anti-IFN- $\gamma$-PE-Cy7, anti-IL-2-Percp-Cy5.5, anti-TNF- $\alpha$-PE, or isotype controls, acquired using multiparameter flow cytometer (BD FACS LSR-II), and analyzed by FACSDiva software.

For toxicity studies, spleens, mLNs and dLNs were collected $18 \mathrm{~h}$ postimmunization to determine the number of different lymphocyte subsets in each treatment group. Cells were incubated with Fc Block, and stained with anti-CD3-FITC, anti-CD8APC-Cy7 and anti-CD4-Alex700, anti- CD11b-PerCp-Cy5.5, anti- CD19-PE-Cy7, antiNK1.1-PE, anti- F4/80-Alexa647, anti- CD11c-PE-Texas Red, or isotype controls followed by acquisition and analysis using multiparameter flow cytometer (BD FACS LSR-II), and FACSDiva software respectively.

For CBA analysis, mice were terminated 3 days after the boost and dLNs were harvested. Cells $\left(2 \times 10^{6}\right.$ cells $\left./ \mathrm{mL}\right)$ were plated triplicate to the wells of 96 well U-bottom plate, and stimulated in vitro with $10 \mu \mathrm{g} / \mathrm{ml}$ of both Her-2/neu ECD protein and p66 peptide for $48 \mathrm{~h}$ at $37{ }^{\circ} \mathrm{C}$. After $48 \mathrm{~h}$ incubation, culture supernatants were harvested and IL-2, IL-4, IL-10, TNF $\alpha$, and IFN $\gamma$ cytokines were determined using Mouse $\mathrm{Th}_{1} / \mathrm{Th}_{2} / \mathrm{Th}_{17}$ Cytokine Cytometric Bead Array Kit and protocols from BD Biosciences. For each assay tube, $10 \mu 1$ aliquot of each capture bead were added and mixed in the same tube. $50 \mu 1$ of mixed cytokine captured beads were mixed with $50 \mu 1$ of sample or standart, and $50 \mu 1$ of PE Detection reagent was added into each assay tube and incubated in the dark at RT for 2h. After incubation, beads were washed with and resuspended in $300 \mu \mathrm{l}$ wash buffer. 
Beads were acquired using multiparameter flow cytometer (BD FACS LSR-II), and analyzed by BD CBA software.

\section{Analysis of Her-2/neu antibodies}

ELISA was performed to assess the presence of anti-Her-2/neu antibodies in vaccinated mice. Briefly, ninety six titer plates coated with $1 \mu \mathrm{g} /$ well of rat Her-2/neu protein for 4 hours at $37^{\circ} \mathrm{C}$ and blocked with PBS containing 5\% BSA $+0.5 \%$ Tween 20 for overnight at $4^{\circ} \mathrm{C}$. Serum dilutions were added to wells and incubated at $37^{\circ} \mathrm{C}$ for 2 hours. Wells were washed 3 times, incubated with anti-mouse IgG-HRP (Jackson Immunoresearch) diluted in blocking buffer for 1 hour at $37^{\circ} \mathrm{C}$, washed, substrate was added and incubated at RT for 30 minutes. Absorbance was measured at $450 \mathrm{~nm}$.

\section{Liver and Kidney functional status:}

C57BL/6 mice were vaccinated with E7 protein $(50 \mu \mathrm{g})+$ either MPL $(25 \mu \mathrm{g})$, or SA-4-1BBL $(25 \mu \mathrm{g})$, or the combination of both agents MPL + SA-4-1BBL $(25 \mu \mathrm{g} /$ agent). Mice were sacrificed 18 hours after vaccination. Serum was collected and status of liver and kidney function was evaluated by serum alanine transaminase (ALT), aspartate transaminase (AST), blood urea nitrogen (BUN), and Creatinin (CREA) levels respectively.

\section{Hematoxylin-eosin staining of liver tissues:}

Mice treated with vaccine formulations as indicated above were terminated $18 \mathrm{~h}$ post immunization, and livers were collected from each treatment groups. PBS vaccinated mice used as negative control. Livers were fixed in $3.7 \%$ formaldehyde, 
embedded in paraffin wax, sliced and stained with Hematoxylin and Eosin for pathological analysis.

\section{Statistical analysis:}

Analysis of variance (ANOVA) was used to compare antibody titers, $\mathrm{CD}^{+}$and $\mathrm{CD}^{+}$effector $\mathrm{T}$ cell responses, and $\mathrm{CBA}$ analysis among experimental groups. Two-tail student t-test was used to compare the number of each lymphocyte population in the spleen, mLN, and dLNs among the experimental groups for toxicity studies. KaplanMeier log-rank test was used to generate the survival curves.

\section{$\underline{\text { Results }}$}

\section{Expression and purification of recombinant rat Her-2/neu ECD}

To generate a recombinant Her-2/neu protein as a TAA for the therapeutic vaccine, we first generated plasmid containing the 6X-His-pTWIN-1 expression vector, carrying the rat Her-2/neu ECD cDNA (Fig. 7.A). The recombinant Her-2/neu was expressed in bacteria, and its expression was induced with IPTG (Fig. 7.B). However, as shown in Fig. 7.B, the protein was degraded. Then, we expressed the rat Her-2/neu ECD cDNA in pTWIN-1-6X-His vector (Fig. 8.A), transfected the bacteria with this construct, and induced the protein expression with IPTG (Fig. 8.B). Although, we were able to express the ECD of rat Her-2/neu protein with the right size (approximately $85 \mathrm{kDa}$, Fig. 8.C), after elution the protein was precipitated out and found to have high endotoxin levels.

To overcome the endotoxin issue, we generated plasmid containing the pMT-BiP expression vector, carrying the rat Her-2/neu ECD cDNA and Flag residues at C- 
terminus (Fig. 9.A). The recombinant Her-2/neu was expressed in Drosophila S2 cells, its expression was induced with $\mathrm{CuSO} 4$ (Fig. 9.B), secreted into the culture supernatant, and purified and eluted using anti-FLAG M2 affinity gel and 3xFLAG peptide, respectively. The purified protein runs approximately as an $85 \mathrm{kDa}$ band on SDS-PAGE (Fig. 9.C), and reacts with anti-ErbB2 antibody on a western blot gel (Fig. 9.D).

Next, we tested if the A2L2 breast cancer cell line that we are planning to use for the tumor studies expresses rat Her-2/neu protein. We evaluated the Her-2/neu expression in the tumor cell lysate. As shown in Fig. 9.E, the whole Her-2/neu (185 kDa) was detected in the A2L2 cell lysate.

\section{SA-4-1BBL adjuvanted rat Her-2/neu TAA-based vaccine induces elimination of established A2L2 tumors in BALB/c mice}

We examined the therapeutic efficacy of SA-4-1BBL in different preclinical tumor models, such as E7 expressing TC-1 tumors [102], and survivin expressing 3LL tumors $[98,107]$. We sought to investigate whether SA-4-1BBL with demonstrated adjuvant activity can generate an anti-tumor efficacy in a more tolerant self-TAA using rat Her-2/neu expressing A2L2 mouse breast cancer model in BALB/c mice. Immunization with rat Her-2/neu as a TAA and SA-4-1BBL as an adjuvant resulted in eradication of A2L2 tumors in 10\% of BALB/c mice in a prime-boost setting. Moreover, mice that expired from tumor in this treatment group had significantly slow tumor progression and prolonged survival as compared to PBS control group ( $<<0.05$; Fig. 10.A-B). We hypothesized that SA-4-1BBL vaccine efficacy could be improved with the recruitment of MPL as a second adjuvant with primary effect on the innate immunity [6, 201]. A prime-boost immunization with rat Her-2/neu protein mixed with SA-4-1BBL + 
MPL resulted in the eradication of A2L2 tumors in $30 \%$ of $\mathrm{BALB} / \mathrm{c}$ mice, which remained TF over an observation period of 90 days. Further, this combined adjuvant treatment resulted in slower tumor progression and prolonged survival significantly as compared to control group ( $<<0.05$; Fig. 10.A-B). In contrast, rat Her-2/neu + MPL monotherapy did not provide protection and all mice expired from the tumor burden within 45 days (Fig. 10.A-B). Taken together, these data demonstrate that SA-4-1BBL + MPL as an adjuvant system is effective in eradicating the established A2L2 tumors with better therapeutic efficacy than the individual agents, and SA-4-1BBL monotherapy has better efficacy than MPL monotherapy.

\section{MPL induced antibody response against Her-2/neu does not correlate with survival}

Since antibody therapies against Her2/neu have been utilized and trastuzumab therapy has demonstrated clinical efficacy [183], generation of humoral response with long lasting antibodies against Her-2/neu ECD may increase the anti-tumor efficacy. Therefore, we collected serum samples from mice with different treatments as indicated above. Serum samples were collected from tumor bearing mice on termination days when tumor size reached $12 \mathrm{~mm}$ in diameter average and from TF mice on day 59, and assessed for anti-Her-2/neu titers. Tumor bearing PBS treated mice did not develop antiHer-2/neu antibody response, while mice vaccinated with Her-2/neu + SA-4-1BBL, and combined adjuvants generated moderate $\mathrm{Ab}$ response, which did not result in statistical significance compared to PBS alone (Fig. 11). Two TF mice in the combination treatment group were negative for anti-Her-2/neu titers while one mouse showed moderate titer against Her-2/neu protein on day 59, and one TF mouse in the SA-4-1BBL monotherapy did not generate antibody response for the target antigen. On the other hand, Her-2/neu + 
MPL vaccination induced significant anti-Her-2/neu titers compared to other treatment groups. However, vaccination with Her-2/neu + MPL did not provide protection against A2L2 tumor challenge.

\section{Vaccination with combined adjuvants and rat Her-2/neu induces better anti-tumor killing response}

$\mathrm{CD}^{+} \mathrm{T}$ cell effector and memory responses is critical for the elimination of primary tumor and control recurrences in various tumor settings [98, 102, 198], and their importance in breast cancer has also been determined in preclinical and clinical studies [194-197]. As such, the number of tumor infiltrating $\mathrm{CD}^{+} \mathrm{T}$ cells is associated with the clinical responsiveness in breast cancer patients and they are critical for the elimination of primary tumor and control of metastasis [194-197]. Therefore, we assessed the antitumor killing responses elicited by vaccine formulations containing rat Her-2/neu and MPL, or SA-4-1BBL, or MPL + SA-4-1BBL. Mice ( $\mathrm{n}=3$ per group) were vaccinated twice with 5 days interval s.c. with the same formulations, and euthanized 5 days later to test the cytotoxic activity of $\mathrm{CD} 8^{+} \mathrm{T}$ cells against rat Her-2/neu expressing $\mathrm{A} 2 \mathrm{~L} 2$ and Her-2/neu negative parental cell line 66.3neo breast cancer cells, (Fig. 12.A). Consistent with the therapeutic efficacy, vaccination with combined adjuvants generated a better A2L2 killing response than single adjuvants, and SA-4-1BBL as monotherapy generated better killing responses than MPL monotherapy (Fig. 12.A). The high cytotoxic killing response against Her-2/neu negative parental cell line 66.3neo in combined adjuvant treatment was not expected (Fig. 12.A). Therefore, we sought to determine the anti-tumor killing response in the combined adjuvant therapy against E7 expressing TC-1 cervical tumor cells to eliminate the possibility of low Her-2/neu expression on the 66.3 cell line 
and killing response against other antigens common for both A2L2 and 66.3 cell lines. We observed that treatment with combined adjuvants did not result in killing of unrelated control TC-1 cervical cancer cells (Fig. 12.B), suggesting that the killing response generated by combined adjuvant therapy is not only Her-2/neu antigen specific, but also specific for other tumor antigens common for both A2L2 and its parental cell line. This might be due to 'epitope spreading'; as vaccine kills tumor cells, they release more tumor associated antigens resulting in generation of immune responses to new epitopes [202].

Vaccination with rat HER-2/neu + SA-4-1BBL induces robust antigen specific cytokine responses in $\mathrm{CD4}^{+}$and $\mathrm{CD8}^{+} \mathrm{T}$ cells

Although the importance of $\mathrm{CD}^{+} \mathrm{T}$ cells has been shown in breast cancer [194196] for the generation of primary $\mathrm{CD} 8^{+} \mathrm{T}$ cell responses and the establishment of longterm memory, $\mathrm{CD}^{+} \mathrm{T}$ cells are also critical to provide help. We, therefore, assessed the $\mathrm{Th}_{1}$, and $\mathrm{CD} 8^{+} \mathrm{T}$ cell effector responses elicited by each vaccine formulations. Mice ( $\mathrm{n}=5$ per group) were primed and boosted s.c. with rat Her-2/neu and either individual agents (MPL or SA-4-1BBL) or combination of both adjuvants with 5 days interval and euthanized 5 days the boost to test the intracellular cytokine response of $\mathrm{CD} 4^{+}$and $\mathrm{CD} 8^{+}$ T cells to the dominant Her-2 ${ }_{66-74}$ CTL epitope in the vaccine dLNs.

While vaccination with rat Her-2/neu + MPL did increase antigen-specific IFN- $\gamma$, IL-2 and TNF- $\alpha$ cytokine production (Fig. 13) in both $\mathrm{CD}^{+}$(upper panel) and $\mathrm{CD} 8^{+}$ (lower panel) $\mathrm{T}$ cells, rat Her-2/neu $+\mathrm{SA}-4-1 \mathrm{BBL}$ therapy evoked much stronger production in these cytokines in both $\mathrm{T}$ cell types (most differences are reaching statistically significance; $\mathrm{p}<0.05$, except for IL-2 responses in both $\mathrm{CD} 4^{+}$and $\mathrm{CD} 8^{+} \mathrm{T}$ cells and IFN- $\gamma$ response in $\mathrm{CD}^{+} \mathrm{T}$ cells, Fig. 13). Interestingly, although vaccination 
with combined adjuvants was more effective than MPL monotherapy in the generation of antigen-specific cytokine responses, it was either equal to (IL-2, and IFN- $\gamma$ in $\mathrm{CD}^{+} \mathrm{T}$ cells) or not as effective as the SA-4-1BBL monotherapy (Fig. 13). Collectively, these

data demonstrate that: i) vaccination with MPL does induce antigen-specific $\mathrm{CD}^{+}$and $\mathrm{CD}^{+}$cytokine responses, ii) SA-4-1BBL monotherapy induces higher cytokine production in both $\mathrm{CD} 4^{+}$and $\mathrm{CD} 8^{+} \mathrm{T}$ cells then MPL monotherapy, and iii) combined adjuvant therapy does not further improve the SA-4-1BBL effect on cytokine responses. Indeed, in the generation of some cytokines, it is less effective than SA-4-1BBL monotherapy.

Vaccination with rat HER-2/neu + SA-4-1BBL increases the number of effector $T$ cell populations in the dLNs of tumor bearing mice

Since we observed the synergistic effect of MPL on the SA-4-1BBL therapy in therapeutic setting (Fig. 10), we sought to determine the anti-tumor mechanism of the vaccine formulations when tumor was in place. For this purpose, mice ( $\mathrm{n}=3$ per group) were inoculated with A2L2 tumors, and when the tumors were around 3-5 mm, mice were primed with the previously indicated vaccine formulations and received the booster dose 5 days later. Three days after the boost, absolute numbers of different effector $\mathrm{CD} 4^{+}$ and $\mathrm{CD}^{+} \mathrm{T}$ cell populations were determined in the dLNs. Vaccination with Her-2/neu + SA-4-1BBL generated higher numbers of effector memory $\left(\mathrm{CD} 44^{\mathrm{hi}} \mathrm{CD} 62 \mathrm{~L}^{\mathrm{lo}}\right)$, resting memory $\left(\mathrm{CD} 44^{\mathrm{hi}} \mathrm{CD} 62 \mathrm{~L}^{\mathrm{hi}}\right)$, and activated $\left(\mathrm{CD} 69^{+}\right) \mathrm{CD}^{+} \mathrm{T}$ cells compared to the PBS vaccinated group (control vs. SA-4-1BBL: $* * *=\mathrm{p}<0.001$ in CD44 ${ }^{\mathrm{hi}} \mathrm{CD} 62 \mathrm{~L}^{\mathrm{lo}} ; *=\mathrm{p}<0.05$ in $\mathrm{CD} 44^{\text {hi }}{ }^{\mathrm{CD}} 62 \mathrm{~L}^{\mathrm{lo}} ; * *=\mathrm{p}<0.01$ in $\mathrm{CD} 69^{+}$cells; Fig. 14), but in doing so it also increased the numbers of these subsets in $\mathrm{CD}^{+} \mathrm{T}$ cells (control vs. SA-4-1BBL: $* * *=\mathrm{p}<0.001$ in 
$\mathrm{CD} 44^{\text {hi }} \mathrm{CD} 62 \mathrm{~L}^{\text {lo }} ; *=\mathrm{p}<0.05$ in $\mathrm{CD} 44^{\mathrm{hi}} \mathrm{CD} 62 \mathrm{~L}^{\text {lo }}$ cells; Fig. 14). On the other hand, MPL monotherapy did not induce the numbers of effector subsets in $\mathrm{CD} 4^{+}$and $\mathrm{CD} 8^{+} \mathrm{T}$ cells as well as SA-4-1BBL monotherapy, although it was significantly higher than PBS vaccinated control group (Fig. 14). Therapy with rat Her-2/neu + both adjuvants did not further increase the number of effector $\mathrm{CD} 4^{+}$and $\mathrm{CD} 8^{+} \mathrm{T}$ cell populations generated by SA-4-1BBL monotherapy in the dLNs. Indeed, it was less effective than SA-4-1BBL monotherapy in $\mathrm{CD}^{+}$effector $\mathrm{T}$ cell populations (Fig. 14).

However, the combined adjuvant therapy induced the highest number of Tregs $\left(\mathrm{CD}^{+} \mathrm{CD} 25^{+} \mathrm{Foxp}^{+}\right)$in the dLNs, which was followed by SA-4-1BBL, and then MPL therapy (Fig. 14). Taken together, SA-4-1BBL monotherapy generates higher numbers of effector $\mathrm{CD}^{+}$and $\mathrm{CD} 8^{+} \mathrm{T}$ cells then MPL monotherapy, and combined adjuvant therapy does not improve the efficacy of SA-4-1BBL in such responses. On the other hand, addition of MPL to SA-4-1BBL adjuvanted vaccine enhances the number of Tregs.

The therapeutic efficacy of SA-4-1BBL + MPL adjuvanted Her-2/neu vaccine is associated with the increased pro-inflammatory and decreased anti-inflammatory cytokine responses

In addition to examining the numbers of effector $\mathrm{T}$ cell subsets along with Tregs, we next determined the functional status of lymphocytes in the setting above mentioned. Vaccination induced changes in pro-inflammatory (IL-2, IFN- $\gamma$, and TNF- $\alpha$ ) and antiinflammatory (IL-4, and IL-10) cytokine production by lymphocytes from the dLNs were determined in the supernatant of $48 \mathrm{hrs}$ cultured cells (Fig 15). SA-4-1BBL + MPL treatment resulted in highest IFN- $\gamma$ production compared to other therapies $(*=p<0.05$ Naive vs. SA-4-1BBL + MPL Fig. 15). However, combined adjuvant therapy did not 
further improve the SA-4-1BBL efficacy in IL-2 and TNF- $\alpha$ production, and production of TNF- $\alpha$ was higher in SA-4-1BBL adjuvanted groups than control or MPL monotherapy. On the other hand, SA-4-1BBL monotherapy resulted in the increased production of IL-4 and IL-10 cytokines leading to a mixed pro and anti-inflammatory response, while addition of MPL decreased the amount of IL-10 $(* *=p<0.01$ SA-41BBL $v s$ SA-4-1BBL + MPL), and IL-4 anti-inflammatory cytokines.

\section{Combined adjuvant therapy with SA-4-1BBL and MPL generates a significant anti- tumor effect in tolerogenic BALB/neuT mice}

It has been demonstrated that treatment with agonistic antibodies against 4-1BB enhances the immune responses in BALB-neuT mice [203-206]. Therefore, we assessed the therapeutic effect of combined adjuvants in a more clinically relevant BALB-neuT model. First of all, we verified the expression of rat Her-2/neu gene (233 bp) in heterozygous female BALB/neuT mice with PCR (Fig. 16) [207], and used the transgenic mice in this study. Eight week old tolerant BALB/neuT mice $(n=5)$ were challenged with A2L2 cells on day 0 and vaccinated with Her-2/neu + SA-4-1BBL + MPL on days 5 and 10, or left untreated (PBS). As shown in Fig. 17, combination of SA-4-1BBL and MPL resulted in eradication of $\mathrm{A} 2 \mathrm{~L} 2$ tumors in $20 \%$ of $\mathrm{BALB} /$ neuT mice, and mice that expired from tumor in this treatment group had significantly slow tumor progression $\left(^{*}=\right.$ $\mathrm{p}<0.05$, Fig. 17.A-B). Since elevated Treg numbers is associated with a poor prognosis of cancer patients $[208,209]$ and depletion of Treg cells results in better immune efficacy of therapeutic vaccines $[210,211]$, we hypothesized that depletion of Treg cells in BALB/neuT mice may further increase the efficacy of combined adjuvant therapy. Therefore, mice $(\mathrm{n}=5)$ were depleted with Treg cells using PC61 mAb two days before 
tumor challenge, and vaccinated with SA-4-1BBL + MPL adjuvanted Her-2/neu vaccine on days 5 and 10 after tumor inoculation. As expected, depletion of Tregs further increased the anti-tumor efficacy of combined adjuvant system, and increased the survival up to $40 \%(* *=p<0.02$, Fig. 17.A-B). Taken together, these data demonstrate that SA-4-1BBL + MPL as an adjuvant system is effective in eradicating the transplanted A2L2 tumors and prolongation of survival of tumor bearing mice, and depletion of Tregs further increase its anti-tumor efficacy.

Therapeutic efficacy of the SA-4-1BBL + MPL adjuvant system is achieved in the absence of detectable toxicity

Toxicity and autoimmunity generated by effective self-TAA-based therapeutic vaccine formulations using potent adjuvants may lead to termination of the studies, and impede future trials [212]. Since, we used combination of two potent adjuvants, SA-41BBL and MPL, for the treatment of breast cancer, we wanted to evaluate the toxicity profile of each vaccine formulation in a more relevant exogenous E7 (TAA) expressing TC-1 model in $\mathrm{Th}_{1}$ biased $\mathrm{C} 57 \mathrm{BL} / 6$ mice, which are more conducive to toxicity studies [213]. C57BL/6 mice ( $\mathrm{n}=4$ per group) were immunized with each vaccine formulations (E7+ MPL, E7 + SA-4-1BBL, or E7 + both adjuvants) and terminated $18 \mathrm{~h}$ postimmunization. Absolute numbers of T cells, B cells, DCs and Macrophages, NK cells and NK T cells in the spleen, $\mathrm{mLN}$, and vaccine draining LNs were determined (Table1). We also evaluated liver and kidney functional status by measuring serum levels of ALT and AST as an indicator of liver function, and BUN and CREA for renal function ( $\mathrm{n}=3$ per group). Although, liver toxicity was reported in the melanoma patients with anti-4-1BB $\mathrm{Ab}$ treatment [81], as shown in Fig. 18, there was no significant difference in these 
enzymes levels in SA-4-1BBL treatment group compared to naïve mice. Moreover, MPL monotherapy or combined adjuvant therapy also did not increase these enzyme levels compared to naïve mice. We further analyzed the liver tissue histology to evaluate the pathology of inflammation in the vaccine treated mice, which appeared normal with no observable differences compared to the liver tissues of naïve mice (Fig. 19). Taken together, these results indicate that combined adjuvant therapy did not generate detectable signs of toxicity, demonstrating the safety profile of this adjuvant system.

\section{$\underline{\text { Discussion }}$}

One out of 5 women with Her-2/neu expressing mammary carcinoma relapses in spite of trastuzumab treatment [214]. To overcome this problem, many active vaccine strategies targeting Her-2/neu have been evaluated in clinical studies [182]. These strategies include peptide and protein based, DNA based, dendritic cell based, and whole tumor cell based vaccines [182]. However, recombinant peptide or protein based vaccines require an adjuvant to generate potent anti-tumor immune responses to target Her-2/neu

protein because of their weak antigenic nature $[11,12]$. In the current study, we tested if the costimulatory ligand SA-4-1BBL and TLR4 agonist MPL with distinct mechanisms of action can serve as a novel adjuvant system for the development of recombinant TAA based breast cancer vaccine. Here we demonstrated that MPL synergized with SA-41BBL as the adjuvant component of rat Her-2/neu TAA-based vaccine to generate better anti-tumor killing response, enhanced pro-inflammatory, and reduced anti-inflammatory cytokine responses that translated into improved therapeutic efficacy in mouse A2L2 
breast cancer model. More importantly, the combined adjuvant therapy induced the eradication of established A2L2 tumors in both $\mathrm{BALB} / \mathrm{c}$ and more tolerant $\mathrm{BALB} / \mathrm{neuT}$ mice, and its efficacy was further improved with the depletion of Tregs in BALB/neuT mice.

In the case of poorly immunogenic tumors, targeting two arms of immunity using TLR ligands and agonists for TNF receptors has been evaluated for the generation of potent anti-tumor immune response. As an example, combined stimulation of TLR-9 and 4-1BB enhances antigen specific CTL responses and induces the rejection of melanoma tumors [215]. Here we particularly focused on TLR4 ligand MPL, as an FDA approved adjuvant, to activate the innate arm of the immune system, and SA-4-1BBL, as a novel adjuvant for the activation of adaptive immunity. MPL interacts with TLR4 on the APCs, and induce their activation. This activation leads to the production of proinflammatory cytokines and upregulation of costimulatory molecules on the cell surface, which in turn, primes adaptive immune responses [6]. However, SA-4-1BBL, binds to the 4-1BB receptor on the activated $\mathrm{CD} 4^{+}$and $\mathrm{CD} 8^{+} \mathrm{T}$ cells, leading to their survival, expansion, acquisition of effector function, and long-term immune memory [90, 199, 200]. More importantly, 4-1BB costimulation has a predominant effect on $\mathrm{CD} 8^{+} \mathrm{T}$ cells that are critical for the elimination of tumors and generation of long term memory as demonstrated in various cancer settings including breast cancer [98, 194-198]. Therefore, here we hypothesized that MPL can co-operate with SA-4-1BBL for the activation of $\mathrm{CD} 8{ }^{+} \mathrm{T}$ cells through APC activation and antigen presentation by APCs [216].

Anti-Her-2/neu antibodies have been detected in breast cancer patients in the early stages, enhanced antibody response was observed when Her-2/neu is overexpressed 
in the primary tumor [183], and antibody treatment against Her-2/neu has shown to be efficacious. Although Trastuzumab has been evaluated and shown to be efficacious, its mechanism of action is not well known. Some possible action mechanisms are: (1) Her2/neu downregulation; (2) reduced signaling of Her-2/neu; (3) inhibition of the shedding of Her-2/neu ECD; (4) blocking proliferation, and induction of apoptosis; (5) angiogenesis inhibition; and finally induction of Antibody-Dependent Cell-Mediated Cytotoxicity (ADCC) [217]. Therefore, in the recombinant protein-based vaccines, induction of humoral immunity to generate long-term anti-Her-2/neu titers along with cellular response might improve the protective efficacy of such vaccines. In this study, we reported that MPL monotherapy induced significant increase in the anti-Her-2/neu titers (Fig. 11), but it failed to provide protection. Even though, all these predicted mechanisms for Trastuzumab can inhibit the cancer cell growth, not all the antibodies are capable of doing so, instead they may stimulate cancer cell growth [218]. It is also possible that these different effects are due to epitope specificity of the antibodies and changes in signaling pathways [218]. Consistent with this, Ma et al. reported that immunization with four different domains of Her-2/neu ECD fused with CD19 scFv induced different IgG Ab levels, and while serum from mice immunized with scFv-D3 induced growth inhibition of SKBR-3 cells despite low titers of $\operatorname{IgG} \mathrm{Ab}$, scFv-D1 immunization did not generate an inhibitory effect despite high anti-Her-2/neu titers [219]. Therefore, anti-Her-2/neu antibodies generated by MPL treatment may not be helpful for tumor eradication. On the other hand, two TF mice in the combination treatment group were negative for anti-Her-2/neu titers while one mouse showed moderate titer against Her-2/neu protein on day 59, and one TF mouse in the SA-4-1BBL 
monotherapy did not generate antibody response for the target antigen. Therefore, further studies such as evaluating the neutralization efficacy of the antibodies must be performed to understand the relation of antibody response with survival.

We did not observe the synergistic effect of MPL on the SA-4-1BBL therapy in terms of cytokine production in $\mathrm{CD}^{+}$and $\mathrm{CD} 8^{+} \mathrm{T}$ cell subsets in the vaccine $\mathrm{dLNs} 5$ days after the boost. Instead, SA-4-1BBL monotherapy induced better cytokine responses than MPL and combined adjuvant therapy (Fig. 13). However, combined adjuvant therapy induced higher numbers of $\mathrm{CD} 4^{+}$and $\mathrm{CD} 8^{+} \mathrm{T}$ cell producing IFN- $\gamma$, TNF- $\alpha$, and IL-2 cytokines than other treatment groups in the spleen (data is not shown). It is possible that cytokine producing effector $\mathrm{T}$ cells in the combined adjuvant therapy have already been migrated into the spleen, and determining such responses 5 days after the boost in the dLNs might be a late time-point. Next, we should investigate the cytokine responses in the dLNs at earlier time points such as 1 or 3 days after the booster immunization to determine the synergistic effect of SA-4-1BBL and MPL combined adjuvant therapy.

Interestingly, in-vitro stimulation with rat Her-2/neu p66 peptide corresponding to $\mathrm{H}-2 \mathrm{~K}^{\mathrm{d}}$-restricted, dominant CTL epitope increased the expression of proinflammatory cytokines in $\mathrm{CD}^{+} \mathrm{T}$ cells when compared to negative control (without p66 peptide stimulation in the in-vitro culture) (Fig. 13). This could be due to the memory response generated by a prime-boost vaccination, since in vivo generated $\mathrm{CD} 8^{+} \mathrm{T}$ cells were ready for a robust antigen specific secondary response in vitro. Therefore, cytokines, such as IFN- $\gamma$ produced by $\mathrm{CD} 8^{+} \mathrm{T}$ cells in vitro, might have induced the production of $\mathrm{Th}_{1}$ cytokines by $\mathrm{CD}^{+} \mathrm{T}$ cells. Further, since NK cells also express 4-1BB, IFN- $\gamma$ produced 
by in vivo activated NK cells may also enhance $\mathrm{CD}^{+} \mathrm{T}$ cell differentiation into $\mathrm{Th}_{1}$ subtype, and production of type I cytokines [220-222].

Although it has been demonstrated that 4-1-BB signaling preferentially activates $\mathrm{CD} 8^{+} \mathrm{T}$ cells over $\mathrm{CD} 4^{+} \mathrm{T}$ cells by us $[102,103]$ and others $[159,160]$, in this study SA4-1BBL monotherapy induced the production of inflammatory cytokines in both $\mathrm{CD} 4^{+}$ and $\mathrm{CD}^{+} \mathrm{T}$ cells (Fig. 13). This could differ between models because Ganguly et al. demonstrated that agonistic anti-4-1BB antibody enhances Gag specific $\mathrm{CD} 4^{+}$but not $\mathrm{CD} 8^{+} \mathrm{T}$ cell responses, while membrane-bound 4-1BBL plasmid DNA induces antigenspecific $\mathrm{CD}^{+}$and $\mathrm{CD} 8^{+} \mathrm{T}$ cell responses in an HIV infection model [175]. Further, these results may provide clinical benefit since $\mathrm{CD} 4^{+} \mathrm{T}$ helper cells significantly contribute to generation of antitumor responses [223-225] by providing essential signals to CTLs directly [226] or through APCs [227, 228].

When tumor was present, MPL worked in synergy with SA-4-1BBL to induce pro-inflammatory cytokine IFN- $\gamma$ production in the lymphocyte cultures from the dLNs (Fig. 15). These results are consistent with the clinical outcome (Fig. 10.A-B), and antitumor killing response (Fig. 12) since IFN- $\gamma$ is required for the induction of MHC class I expression which is critical for the antigen specific CTL killing of the cancer cells [229]. On the other hand, immunization with Her-2/neu + SA-4-1BBL also induced antiinflammatory cytokine IL-10, and IL-4 production, suggesting a mixed response generated by SA-4-1BBL. Addition of MPL to SA-4-1BBL adjuvanted vaccine decreased the IL-10, and IL-4 production, and shifted the immune response towards inflammatory response. However, IL-10 effect on the immune system has been controversial since it can suppress the inflammatory cytokine production, while, in doing 
so, it can induce CTL and NK cytotoxic activity [230-232]. Furthermore, its impact on tumor growth has also been controversial since it has been shown that IL-10 suppresses tumor growth and inhibits metastasis in several models, including breast cancer [233236], while it promotes tumor growth through Stat3 activation [237]. Nevertheless, both pro- and anti-inflammatory activity of IL-10 has been shown to be important in rejection of melanoma tumors [238]. As a result, the enhanced anti-tumor efficacy by combined adjuvant therapy might be due to the generation of a more balanced anti- and proinflammatory response.

Tregs are the critical players in the escape mechanism of cancer cells from the immune system attack [239], and they have a critical effect in tumor progression in different tumor models, including breast cancer [208, 240, 241]. Moreover, the number of intratumoral Tregs is a prognostic predictor in breast cancer patients [242, 243]. Therefore, in cancer immunotherapy, controlling the number and/or function of Tregs is another critical aim for better vaccine efficacy. As such, depletion of Tregs using antibodies or cyclophosphamide has been shown to improve the anti-tumor efficacy in preclinical models [244-247]. Consistent with preclinical studies, breast cancer patients with lower numbers of Tregs infiltrated into the tumor after neoadjuvant chemotherapy was associated with higher pathological response rates [243]. Our data further supports the role of Tregs in the vaccine efficacy since their depletion resulted in increased survival in BALB/neuT mice treated with combined adjuvants (Fig. 17.A-B). However, the difference was not statistically significant. This could be due to the timing, schedule of administration or dose of PC-61 antibody since we only used one dose of PC-61 at one time point. On the other hand, the augmented numbers of Tregs in the dLNs of the 
treatment groups with better therapeutic efficacy was not expected. Combined adjuvant therapy induced the highest numbers of Tregs, which was followed by SA-4-1BBL, and MPL therapy (Fig. 14). However, our data do not contradict with previously published studies by us using SA-4-1BBL and others using agonistic anti-4-1BB antibody where 41BB costimulation enhances the proliferation of Tregs [101, 248]. It is likely that SA-41BBL renders $\mathrm{T}$ effector cells resistant to Treg suppression within draining lymph nodes and tumor $[98,101]$, or the high number of infiltrated Tregs in tumor draining lymph nodes may be a trafficking issue [72]. Therefore, the increased numbers of Tregs in the vaccine dLNs may not be a limiting factor for the efficacy of the SA-4-1BBL adjuvanted vaccines. On the other hand, it has been shown that TLR-4 is also expressed on $\mathrm{CD}^{+} \mathrm{T}$ effector and Tregs [249, 250], and stimulation with LPS induces Treg survival, expansion, and enhances their regulatory function in vivo [250], which might be the reason for increased number of Tregs in the dLNs of MPL treated group. Further studies must be performed to look at the Treg numbers within the tumor, to evaluate whether SA4-1BBL adjuvanted vaccines may suppress the trafficking of Tregs into the tumor.

Costimulatory molecules have a critical role in generating protective cellular immune responses [70, 200]. Agonistic antibodies to costimulatory members of CD28 and TNFR families are effective in generating immune responses against cancer and infectious agents [251-253]. However, agonistic antibodies can cause severe toxicity due to nonspecific lymphocyte activation systemically $[103,254]$, and toxicity generated by vaccination is a critical limiting factor. Towards this goal, we have developed the costimulatory molecule $4-1 \mathrm{BBL}$ in a novel soluble form as a novel adjuvant. As we reported previously SA-4-1BBL generates more potent and qualitatively different 
immunostimulatory activity without detectible toxicity as assessed by systemic cytokine response, non-specific lymphoproliferation, lymphomegaly and splenomegaly, and hepatitis compared to the agonistic 4-1BB mAb [103]. In this study, we further investigated the possible induction of toxicity by two potent immunomodulators. Importantly, the therapeutic efficacy of SA-4-1BBL + MPL adjuvant system was achieved in the absence of detectable toxicity as assessed by various indicators, such as total number of various lymphocyte populations in dLNs, mLNs and spleen (Table 1), liver and kidney enzyme levels to determine functional status of these organs (Fig. 18), and histological analysis of liver to measure inflammation (Fig. 19). The lack of toxicity in SA-4-1BBL monotherapy is consistent with our previously published study where treatment of mice with 4-fold higher SA-4-1BBL over the therapeutic dose used in this study did not induce toxicity [103]. MPL has also been shown to be safe in preclinical and clinical studies $[6,201,255]$, and our results further supports the safety of MPL adjuvanted vaccine. Therefore, the lack of detectable toxicity in the combined and individual adjuvant therapies provides another advantage along with therapeutic efficacy for the human use.

In conclusion, here we demonstrate the efficacy of the SA-4-1BBL + MPL adjuvant system in inducing potent anti-tumor killing responses and antigen specific production of IFN- $\gamma$, a signature cytokine for CTL killing activity, and suppressing the anti-inflammatory IL-10 production by generating a more balanced pro- and antiinflammatory environment compared to SA-4-1BBL monotherapy which translate into potent therapeutic efficacy in Her-2/neu expressing A2L2 breast cancer model. Moreover, depletion of Tregs further increases the therapeutic efficacy of the adjuvant 
system in tolerant BALB/neuT mice. Importantly, the therapeutic efficacy of the vaccines was observed in the absence of detectable toxicity which was tested in E7 expressing TC1 model. Enhancing the efficacy of individual adjuvants with the combined adjuvant therapy emphasizes the importance of combining the adjuvants with different action mechanisms for a better therapeutic efficacy. Finally, this combined adjuvant therapy can be utilized for other cancers, and infectious diseases for a better efficacy. 

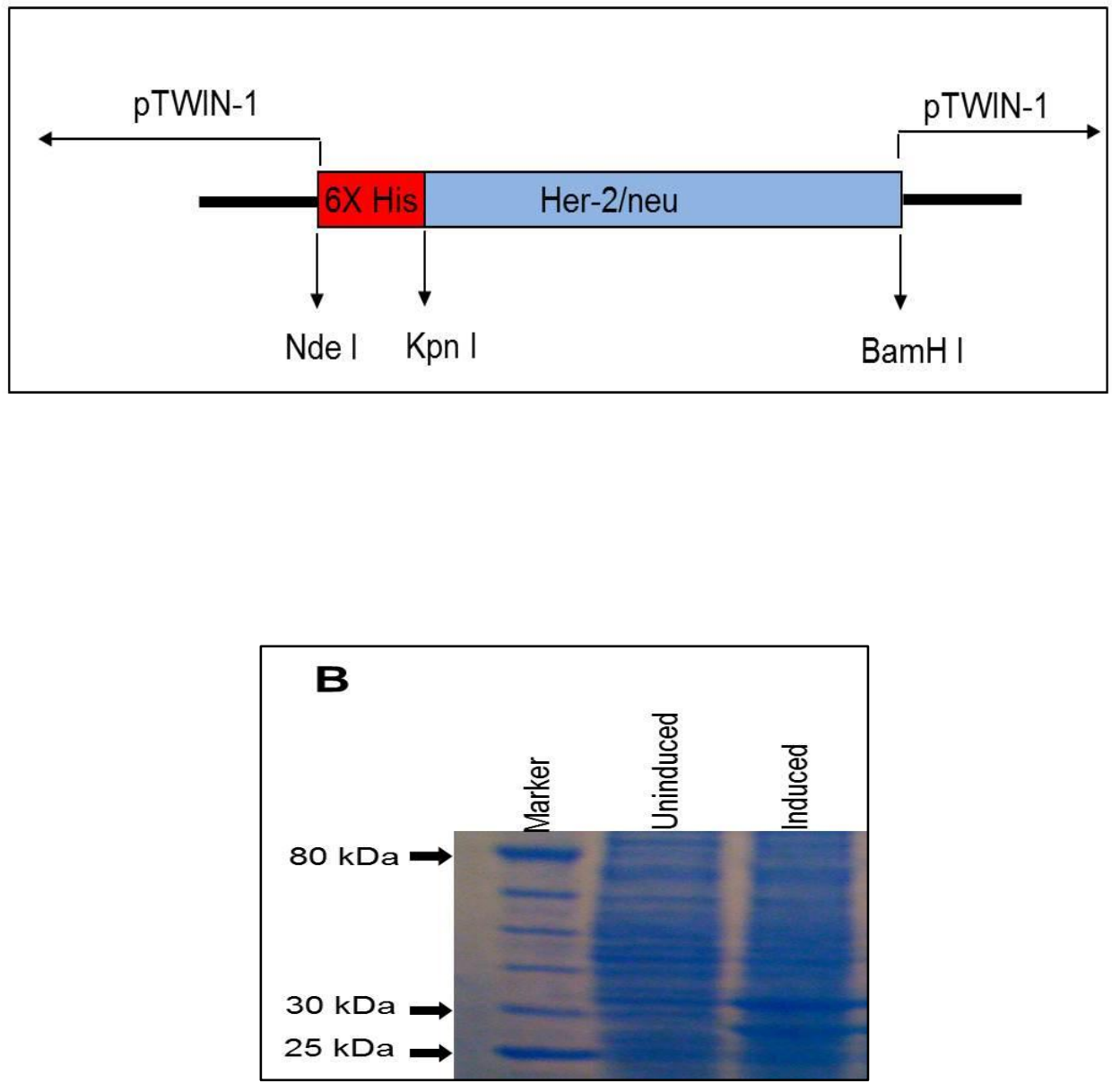

\section{Figure 7}

Production of recombinant rat Her-2/neu ECD in bacteria. A) Schematic representation of 6X-His-pTWIN-1 expression vector with Her-2/neu. B) Coomassie blue staining of uninduced and induced samples with IPTG. 

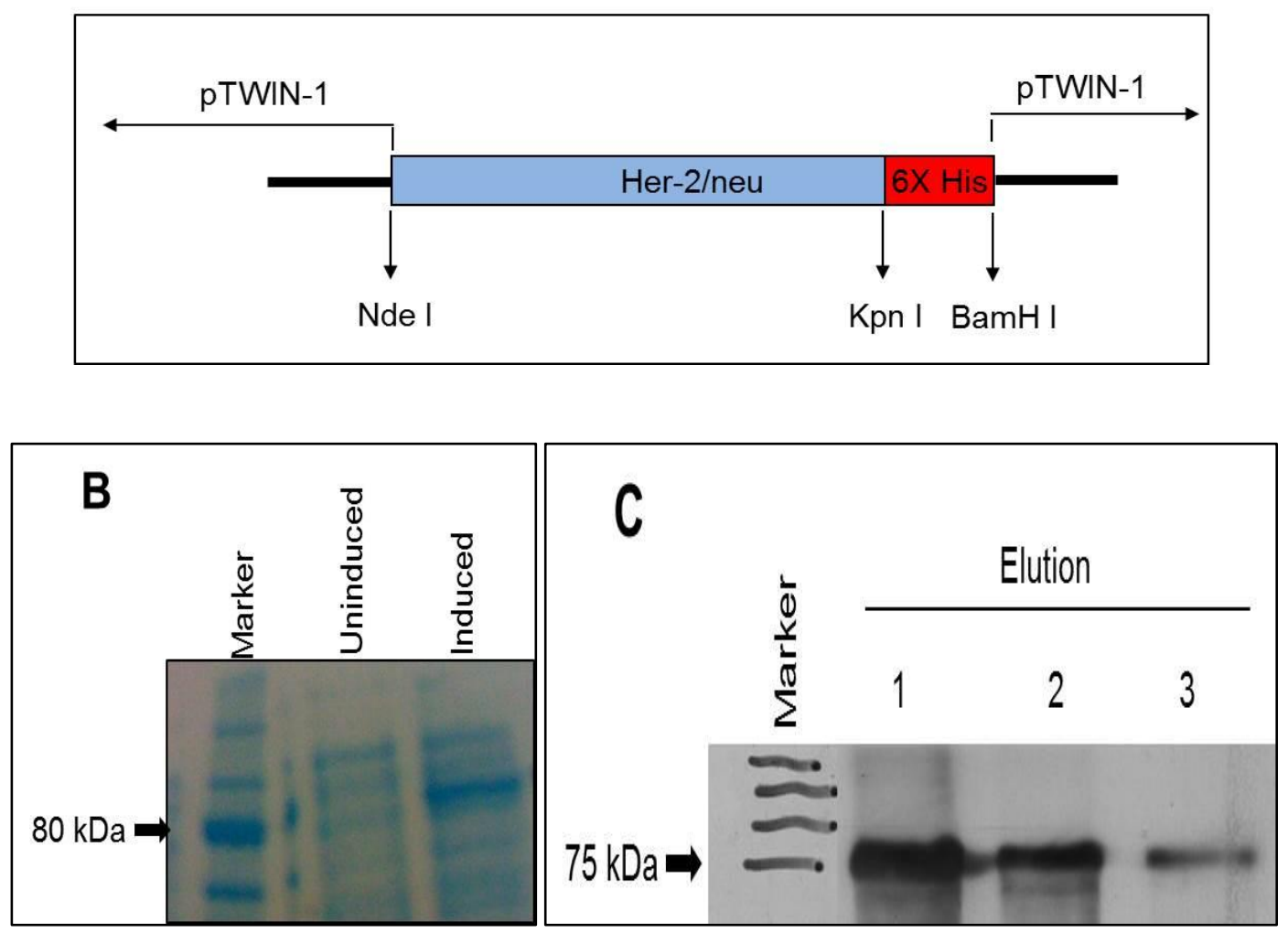

\section{Figure 8}

Production of recombinant rat Her-2/neu ECD in bacteria. A) Schematic representation of pTWIN-1- 6X-His expression vector with Her-2/neu. B) Coomassie blue staining of uninduced and induced samples with IPTG. C) Western blot picture of recombinant rat Her-2/neu protein as detected by anti-ErbB2 antibody. 
A
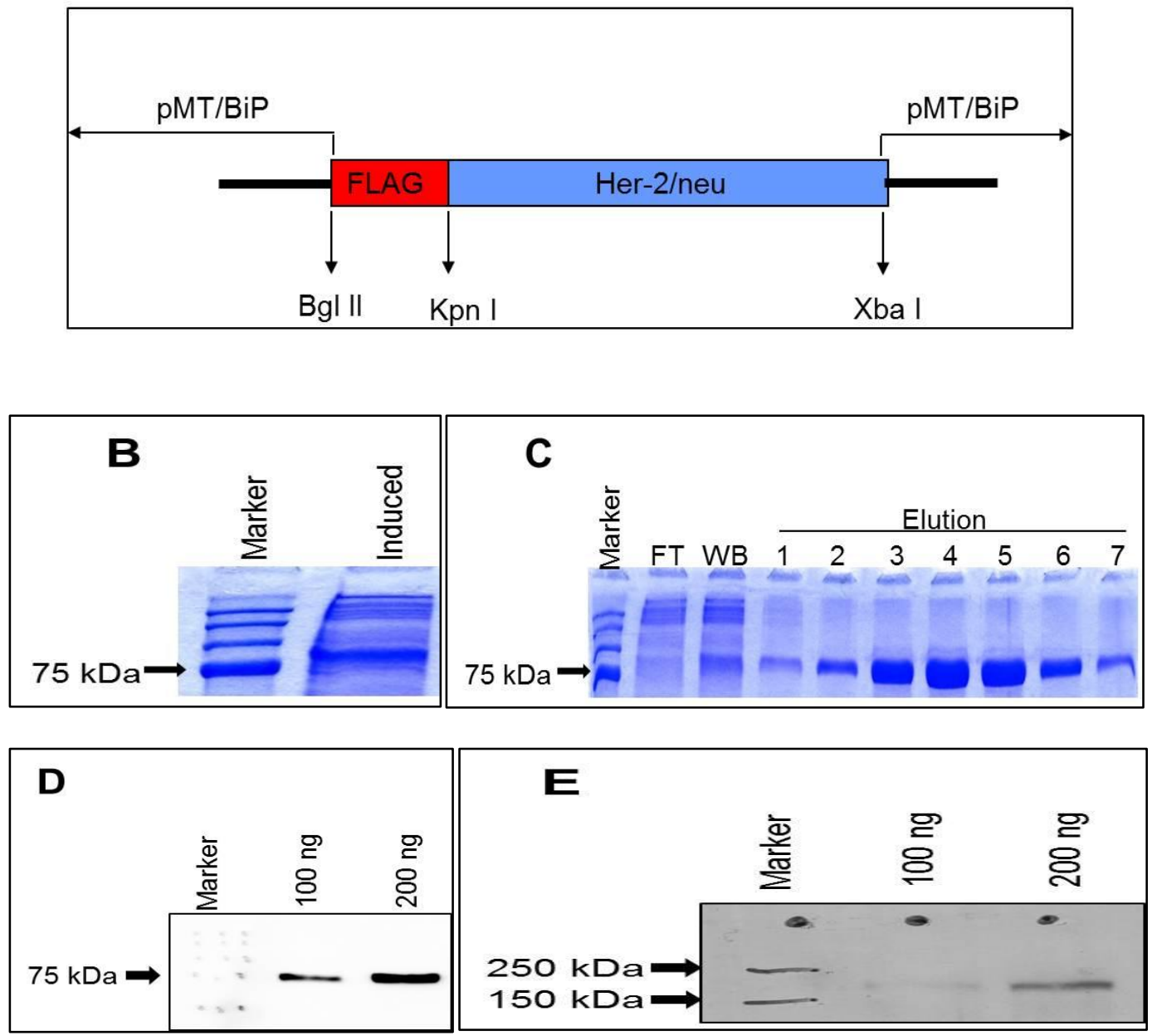

\section{Figure 9}

Production of recombinant rat Her-2/neu protein ECD in Drosophila S2 cells, and Her2/neu expression in A2L2 cells. A) Schematic representation of $\mathrm{pMT} / \mathrm{BiP}$ expression vector with Her-2/neu. B) Coomassie blue staining of $\mathrm{CuSO}_{4}$ induced samples. C) Purified rat Her-2/neu pattern (FT: flow through, WB: wash buffer). D) Western blot picture of recombinant rat Her-2/neu protein. E) Whole Her-2/neu protein expression in A2L2 cell lysate as evaluated by anti-ErbB2 antibody on western blot. 


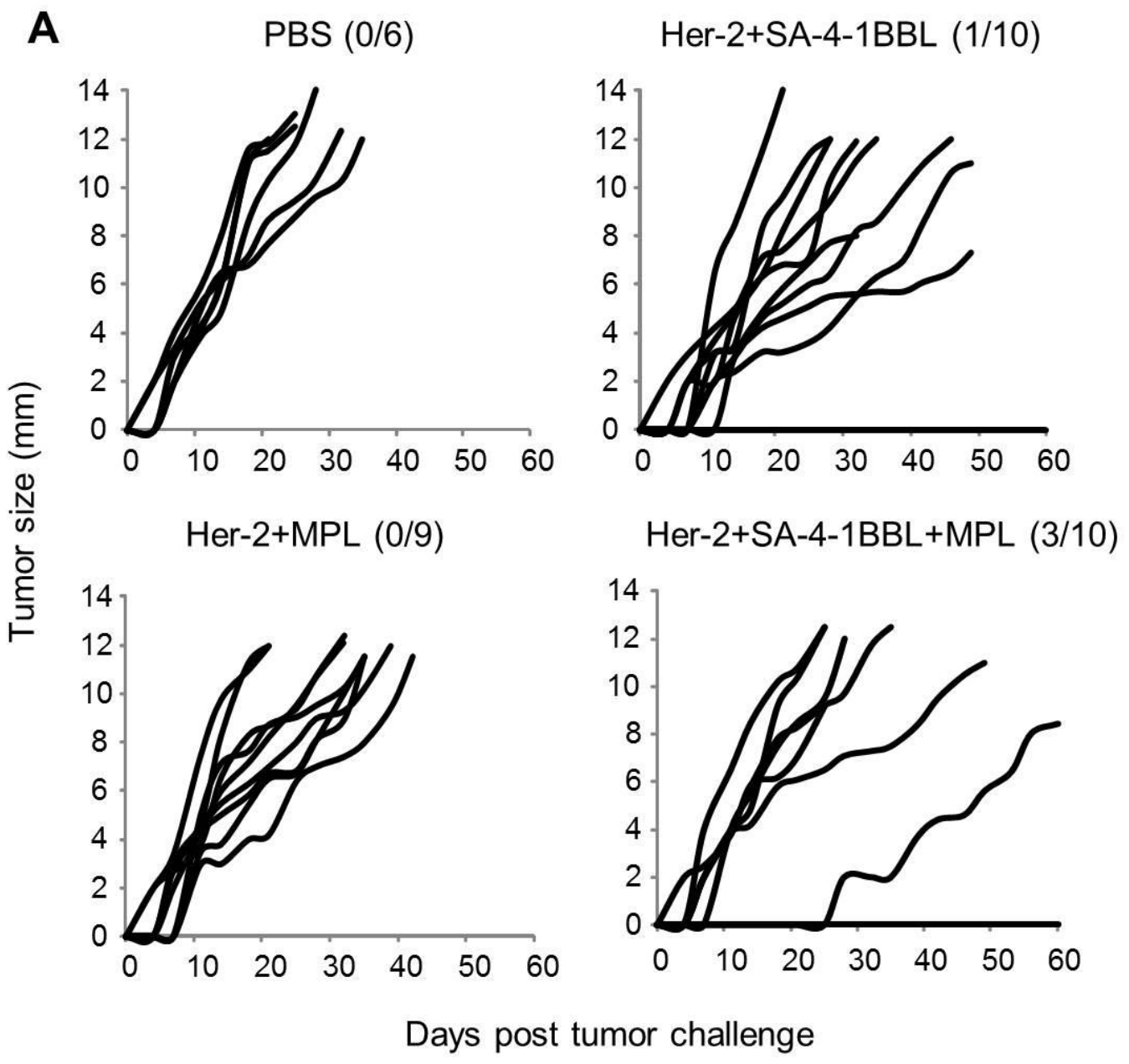


B

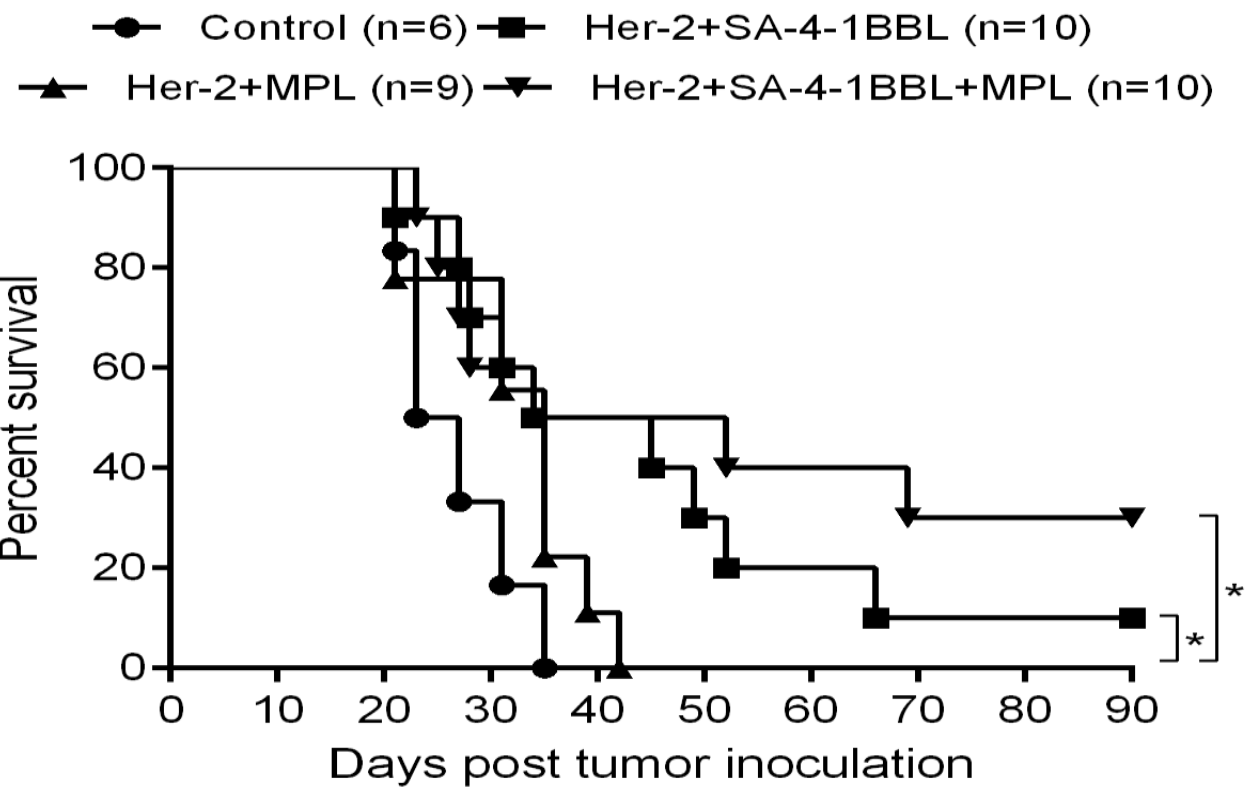

\section{Figure 10}

A prime-boost vaccination with SA-4-1BBL and MPL adjuvant system results in eradication of established A2L2 tumors. BALB/c mice were challenged s.c. with $1 \times 10^{5}$ live rat Her-2/neu expressing A2L2 tumor cells and left untreated (PBS) or vaccinated twice s.c. on days 5 and 10 post-tumor challenge with rat Her-2/neu $(50 \mu \mathrm{g})$ mixed with MPL $(25 \mu \mathrm{g})$, or SA-4-1BBL $(25 \mu \mathrm{g})$, or combination of both agents (25 $\mu \mathrm{g} /$ agent $)$. A) Individual tumor growths in each treatment group. B) Survival comparison of treatment groups. $*=p<0.05$. 


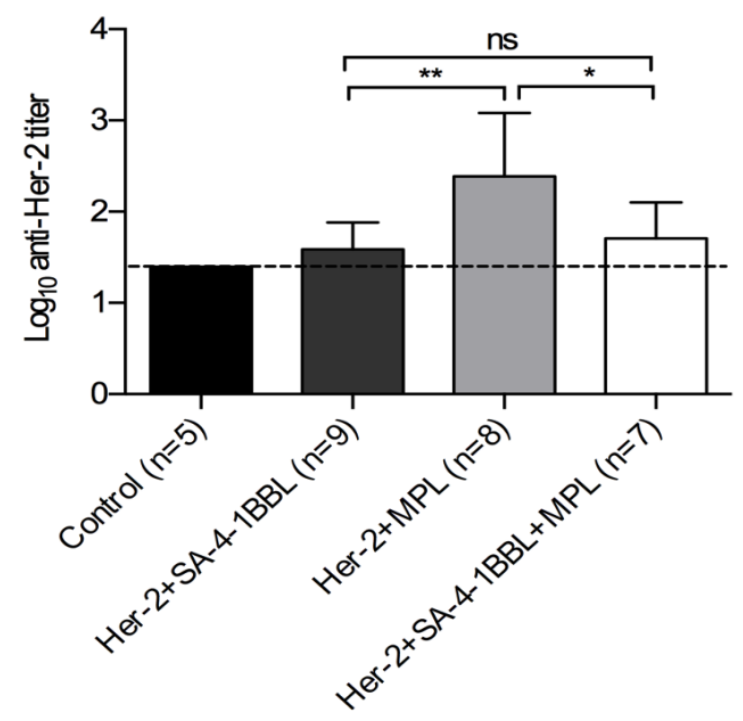

Figure 11

A prime-boost vaccination with Her-2/neu + MPL induces significant antibody response. Serum was collected from each treatment groups as indicated above on termination day of mice when tumor was around $12 \mathrm{~mm}$ diameter or on day 59 when tumor was eradicated. Anti-Her-2/neu titers were determined by ELISA. Bar graph shows the average log titers of anti-Her-2/neu in each treatment group. Dotted line represents the limit of detection. $*=\mathrm{p}<0.02 ; * *=\mathrm{p}<0.01$. 
A
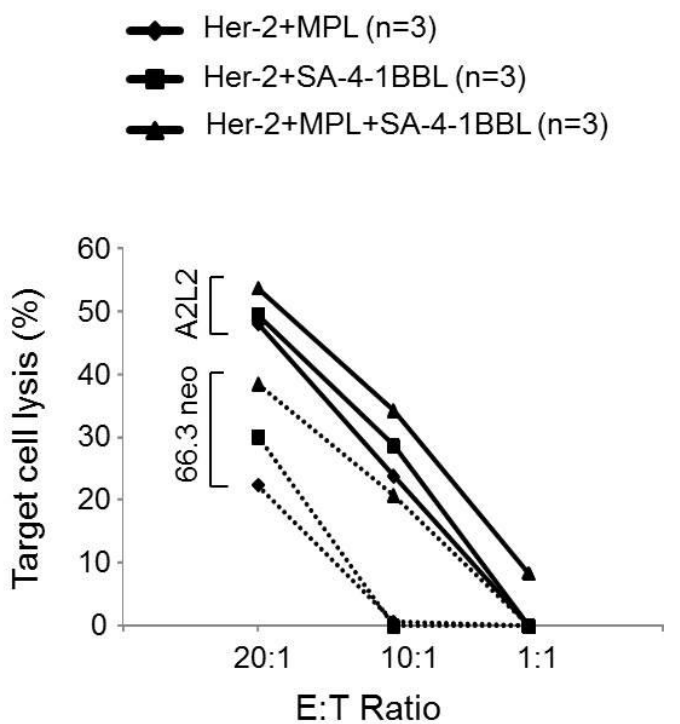

B

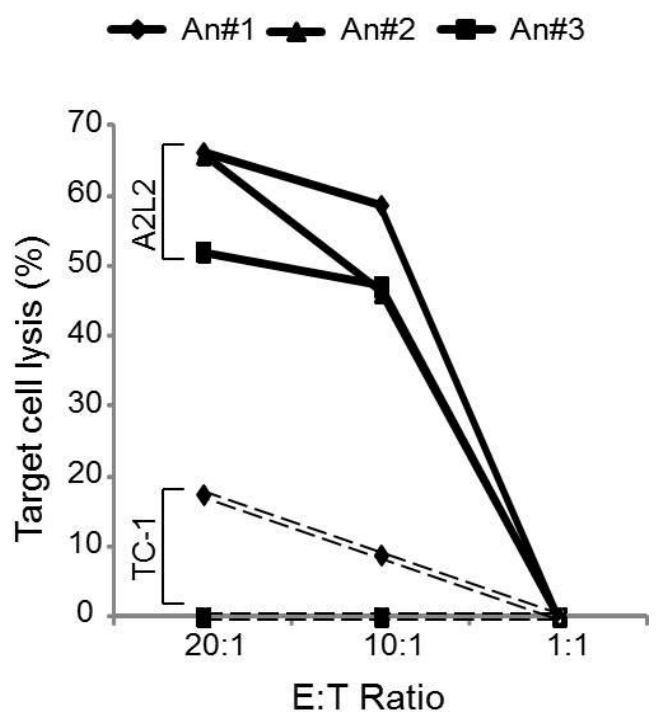

\section{Figure 12}

SA-4-1BBL + MPL adjuvant system induces a strong tumor-specific killing response. BALB/c mice were vaccinated twice with 5 days interval with indicated formulations. 5 days after booster dose injection, lysis of target cells were determined. A) Tumor specific lysis of A2L2 and 66.3 neo target cells. B) Tumor specific (A2L2) and non-specific (TC1) target cell lysis were determined in mice treated with combined adjuvants. 

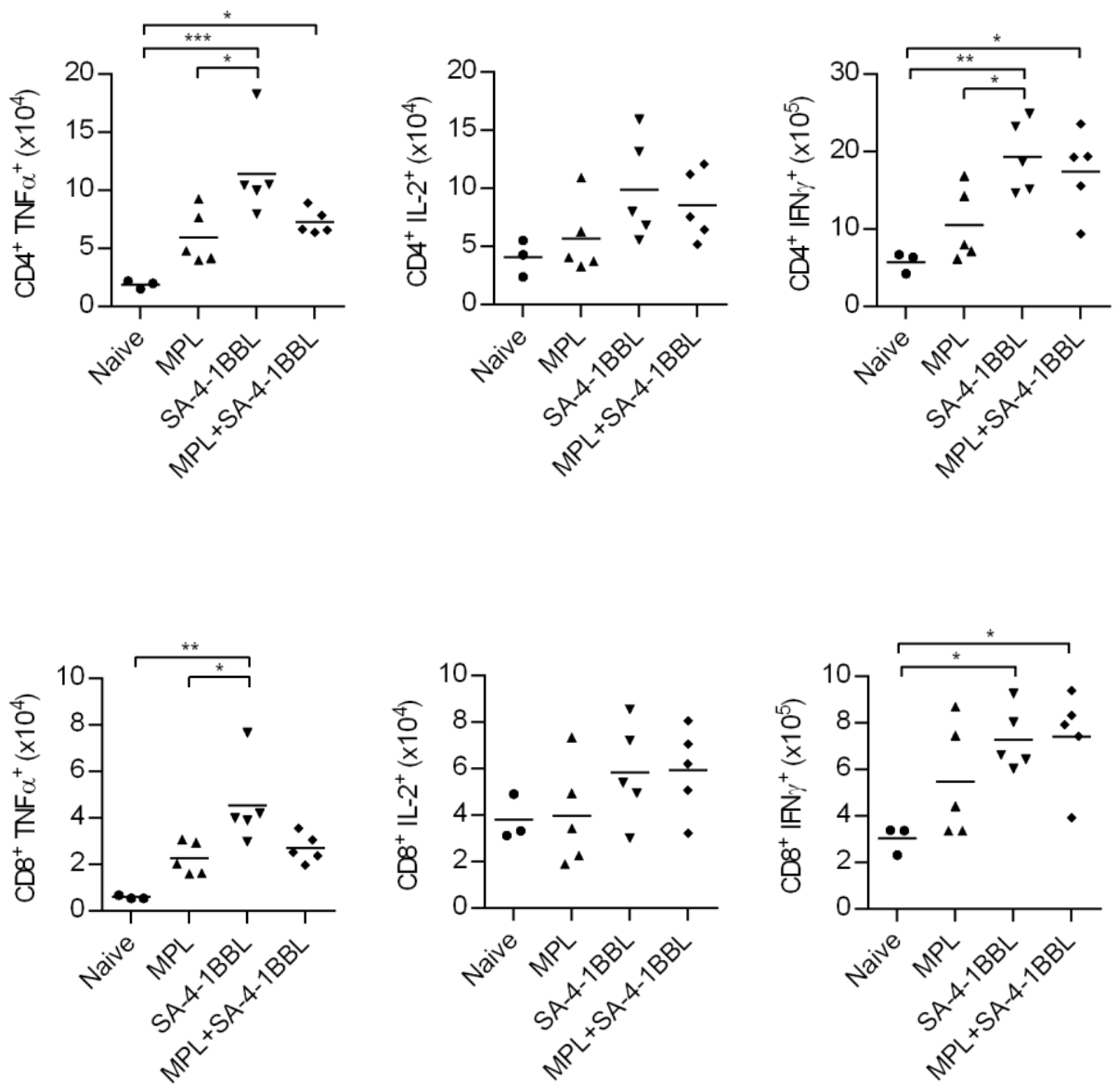

\section{Figure 13}

SA-4-1BBL based Her-2/neu vaccine induces a strong cytokine response. BALB/c mice were vaccinated twice with 5 days interval with Her-2/neu and indicated adjuvants. 5 days after the boost, peptide specific cytokine responses were determined in the dLNs. Each graph represents the individual numbers of $\mathrm{CD} 4{ }^{+} \mathrm{CD} 44^{\text {hi }}$ (Upper panel) and $\mathrm{CD}^{+} \mathrm{CD} 44^{\text {hi }}$ (lower panel) $\mathrm{T}$ cells producing TNFa, IL-2, and IFNy cytokines. Horizontal line represents the mean value of each group. ${ }^{* * *}=\mathrm{p}<0.001 ; * *=\mathrm{p}<0.01 ; *$ $=\mathrm{p}<0.05$. 

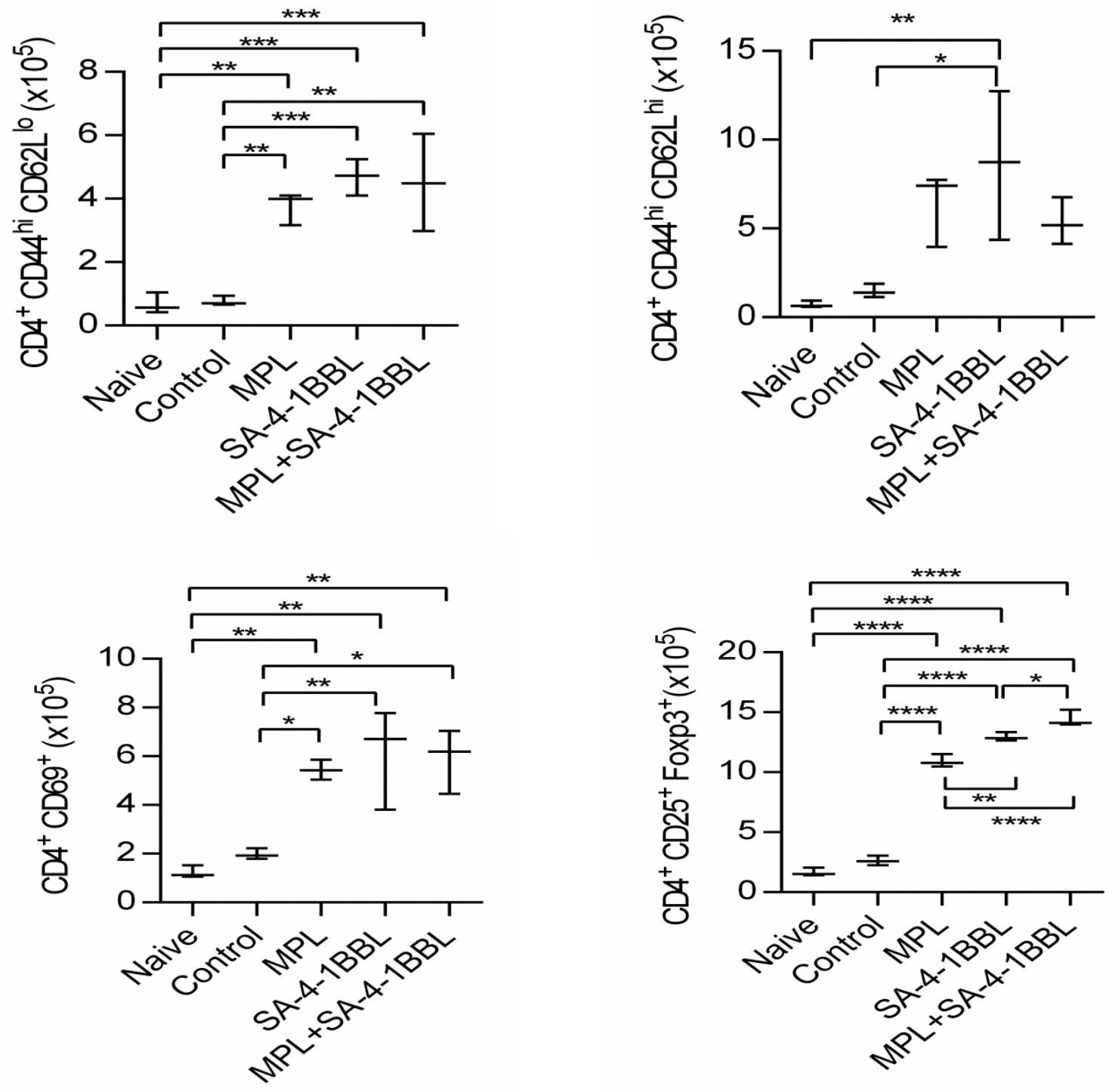

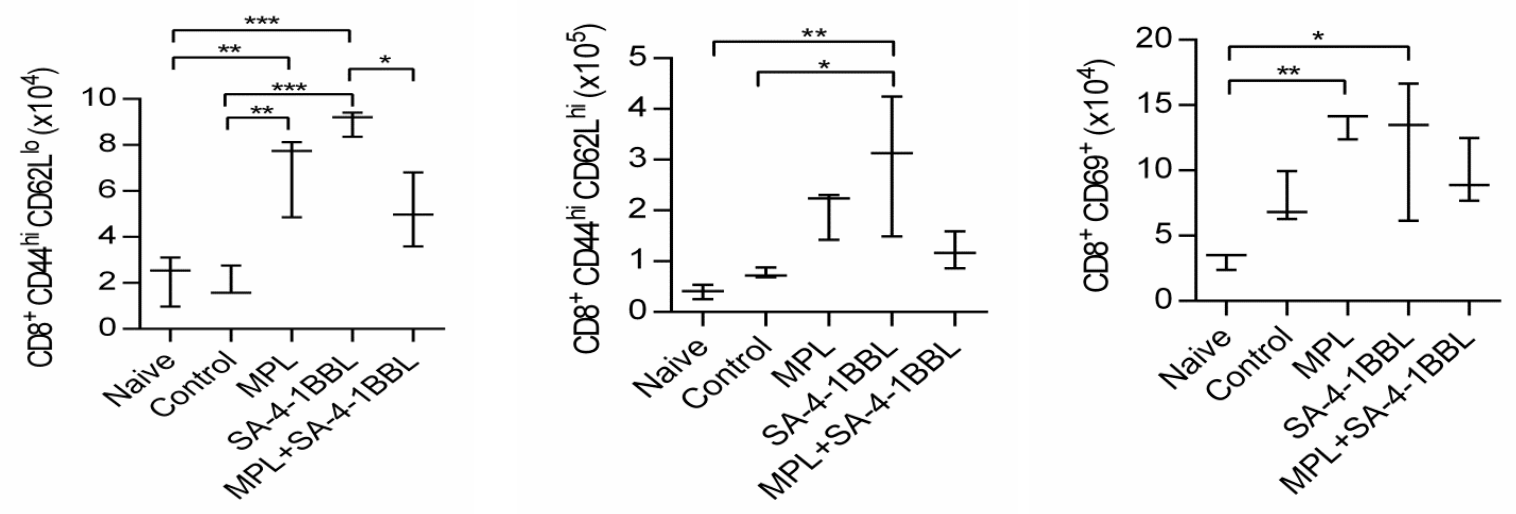

\section{Figure 14}

Vaccination with Her-2/neu + SA-4-1BBL induces high numbers of effector $\mathrm{T}$ cell subsets in the dLNs of tumor bearing mice. Tumor bearing $(3-5 \mathrm{~mm}) \mathrm{BALB} / \mathrm{c}$ mice were vaccinated twice with 5 days interval. 3 days after the boost, absolute numbers of different $\mathrm{CD}^{+}$and $\mathrm{CD}^{+} \mathrm{T}$ cell populations were determined. Each bar represents the mean and S.D. of absolute numbers of $\mathrm{T}$ cell subsets in each treatment group. $* * * *=$ $\mathrm{p}<0.0001 ; * * *=\mathrm{p}<0.001 ; * *=\mathrm{p}<0.01 ; *=\mathrm{p}<0.05$ 

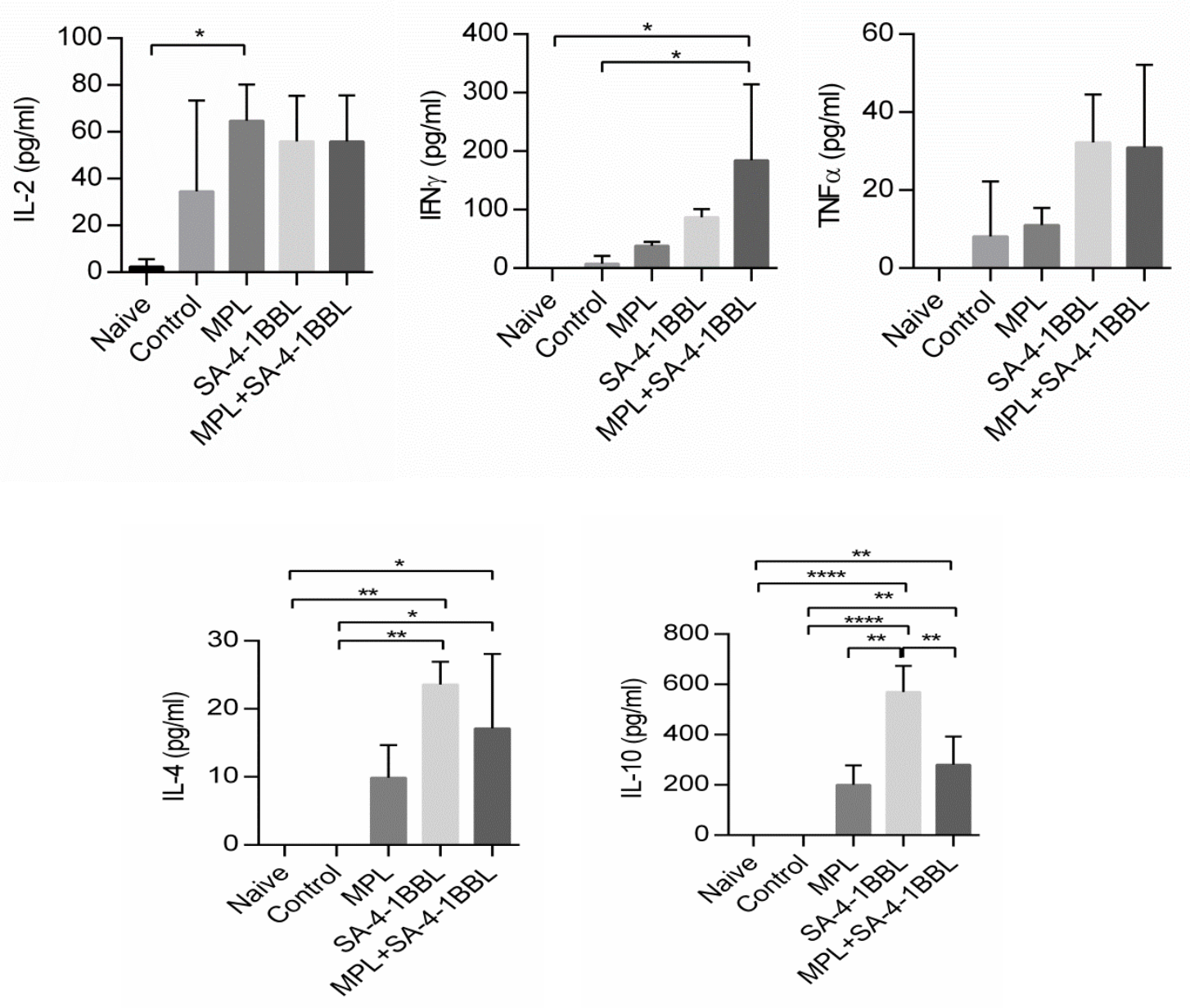

\section{Figure 15}

Addition of MPL to the SA-4-1BBL adjuvanted vaccine increases pro-inflammatory and decreases anti-inflammatory cytokine production in the dLNs of tumor bearing mice. Tumor bearing $(3-5 \mathrm{~mm}) \mathrm{BALB} / \mathrm{c}$ mice were vaccinated twice with 5 days interval. 3 days after the boost, lymphocytes from the dLNs were cultured in the presence of Her2/neu protein and p66 peptide for $48 \mathrm{~h}$. Culture supernatants were collected and cytokine responses were determined by CBA analysis. Each bar represents the mean and S.D. of cytokine amount in each treatment group. $* * * *=\mathrm{p}<0.0001 ; * *=\mathrm{p}<0.01 ; *=\mathrm{p}<0.05$. 


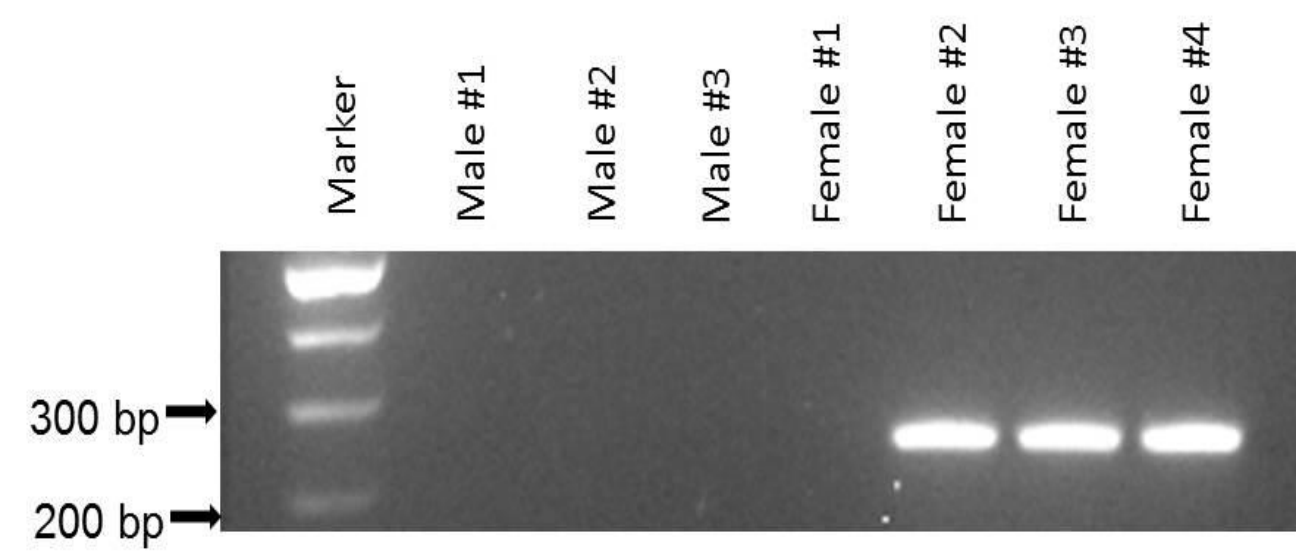

Figure 16

Verification of the expression of rat Her-2/neu gene in BALB/neuT mice with PCR. DNA gel picture of Her-2/neu gene (233 bp) in female BALB/neuT mice. 


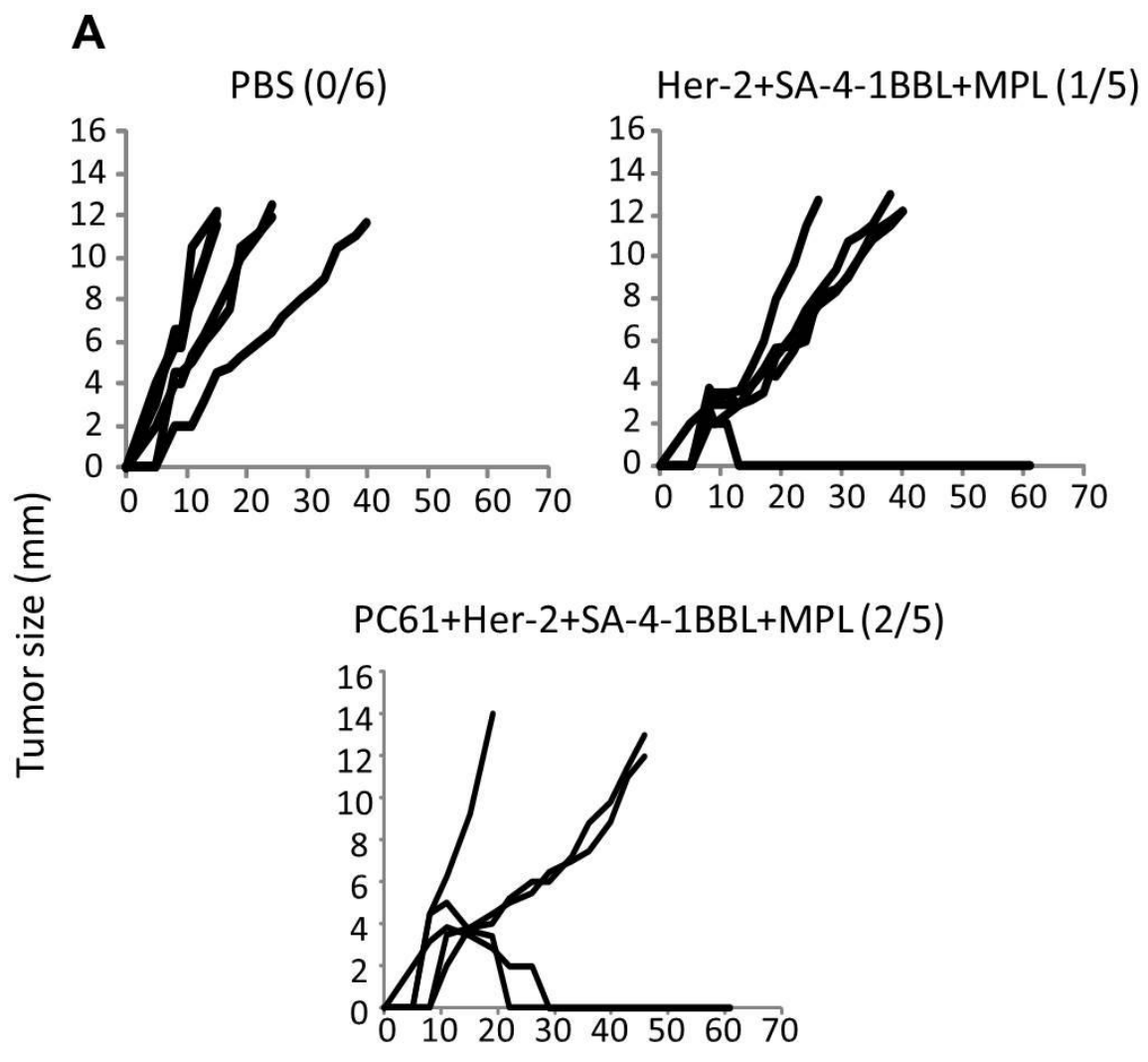

Days post tumor challenge 
B

$\rightarrow$ Control $(n=6) \multimap$ Her-2+SA-4-1BBL+MPL $(n=5)$

$\rightarrow$ PC-61+Her-2+SA-4-1BBL+MPL $(n=5)$

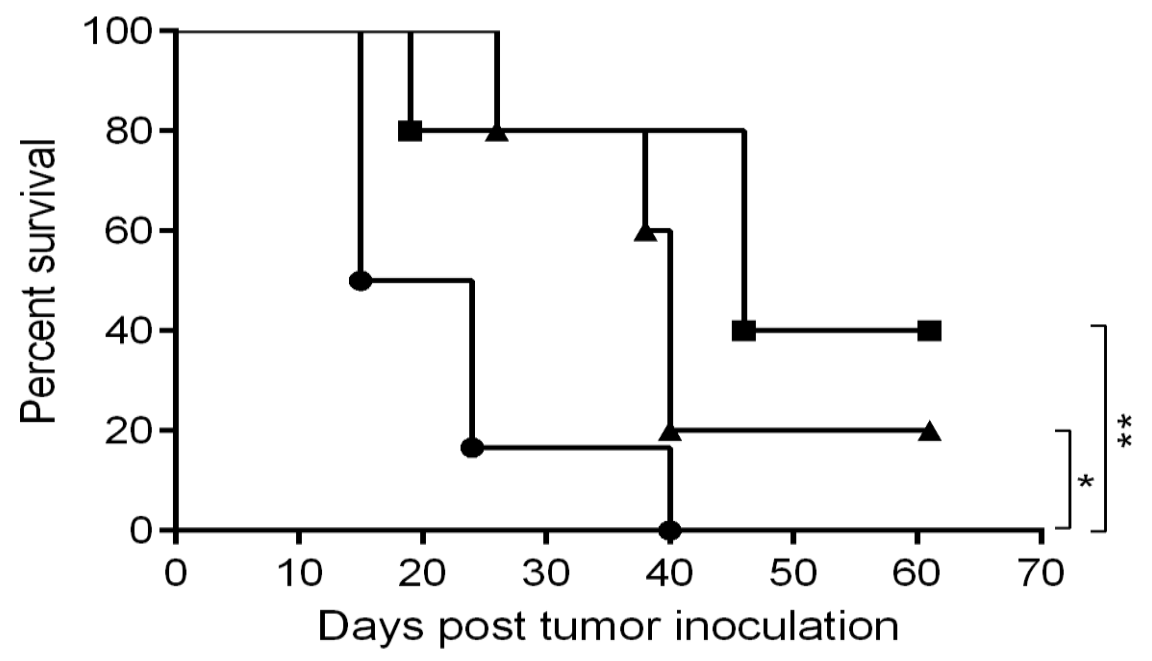

\section{Figure 17}

While treatment with SA-4-1BBL and MPL adjuvanted vaccine results in eradication of established A2L2 tumors in $20 \%$ of BALB/neuT mice, depletion of Tregs further improves the vaccine efficacy. BALB/neuT mice were challenged s.c. with $1 \times 10^{5}$ live rat Her-2/neu expressing A2L2 tumor cells and left untreated (PBS) or vaccinated twice s.c. on days 5 and 10 post-tumor challenge with rat Her-2/neu $(50 \mu \mathrm{g})$ mixed with MPL (25 $\mu \mathrm{g})$ and SA-4-1BBL $(25 \mu \mathrm{g})$, or first treated with PC-61 depleting mAb (300 $\mu \mathrm{g})$ two days before tumor inoculation and then treated with the combined adjuvant therapy. A) Individual tumor growths of mice in each treatment group. B) Survival comparison of treatment groups. $*=\mathrm{p}<0.05 ; * *=\mathrm{p}<0.02$ 


\section{A. Total numbers of lymphocytes in the spleen}

\begin{tabular}{|c|c|c|c|c|c|c|c|}
\hline & $\begin{array}{l}\mathrm{CD4}^{+} \mathrm{T} \\
\text { cells }\end{array}$ & $\begin{array}{c}\mathrm{CD8}^{+} \mathrm{T} \\
\text { cells }\end{array}$ & B cells & NK cells & NK T cells & $\begin{array}{l}\text { Macropha } \\
\text { ges }\end{array}$ & DCs \\
\hline Naive & $\begin{array}{l}22 \pm 2 \\
\left(\mathrm{x} 10^{6}\right)\end{array}$ & $\begin{array}{l}12 \pm 2 \\
\left(\mathrm{x} 10^{6}\right)\end{array}$ & $44 \pm 4\left(\times 10^{6}\right)$ & $2 \pm 0.3\left(\mathrm{x} 10^{6}\right)$ & $\begin{array}{c}0.5 \pm 0.2 \\
\left(\mathrm{x} 10^{6}\right)\end{array}$ & $\begin{array}{c}0.4 \pm 0.1 \\
\left(\mathrm{x} 10^{6}\right)\end{array}$ & $6 \pm 1\left(\times 10^{6}\right)$ \\
\hline E7+MPL & $\begin{array}{l}20 \pm 3 \\
\left(\times 10^{6}\right)\end{array}$ & $\begin{array}{c}11 \pm 2 \\
\left(x 10^{6}\right)\end{array}$ & $\begin{array}{l}44 \pm 12 \\
\left(\mathrm{x} 10^{6}\right)\end{array}$ & $\begin{array}{c}0.9 \pm 0.2 \\
\left(\mathrm{x} 10^{6}\right)(* *)\end{array}$ & $\begin{array}{c}0.5 \pm 0.2 \\
\left(\mathrm{x} 10^{6}\right)\end{array}$ & $\begin{array}{c}0.3 \pm 0.2 \\
\left(\mathrm{x} 10^{6}\right)\end{array}$ & $8 \pm 2\left(\times 10^{6}\right)$ \\
\hline $\begin{array}{c}\text { E7+SA-4- } \\
\text { 1BBL }\end{array}$ & $\begin{array}{l}22 \pm 2 \\
\left(\mathrm{x} 10^{6}\right)\end{array}$ & $\begin{array}{c}12 \pm 2 \\
\left(\mathrm{x} 10^{6}\right)\end{array}$ & $47 \pm 3\left(\times 10^{6}\right)$ & $1 \pm 0.1\left(\mathrm{x} 10^{6}\right)$ & $\begin{array}{c}0.5 \pm 0.1 \\
\left(\mathrm{x} 10^{6}\right)\end{array}$ & $\begin{array}{l}0.2 \pm 0.06 \\
\left(\mathrm{x} 10^{6}\right)(*)\end{array}$ & $8 \pm 1\left(\times 10^{6}\right)$ \\
\hline $\begin{array}{c}\text { E7+MPL+S } \\
\text { A-4-1BBL }\end{array}$ & $\begin{array}{c}21 \pm 4 \\
\left(\times 10^{6}\right)\end{array}$ & $\begin{array}{c}12 \pm 2 \\
\left(\mathrm{x} 10^{6}\right)\end{array}$ & $\begin{array}{l}52 \pm 10 \\
\left(\times 10^{6}\right)\end{array}$ & $2 \pm 0.5\left(\times 10^{6}\right)$ & $\begin{array}{c}0.7 \pm 0.2 \\
\left(\mathrm{x} 10^{6}\right)\end{array}$ & $\begin{array}{c}0.4 \pm 0.1 \\
\left(\mathrm{x} 10^{6}\right)\end{array}$ & $8 \pm 1\left(\times 10^{6}\right)$ \\
\hline
\end{tabular}

\section{B. Total numbers of lymphocytes in the dLNs}

\begin{tabular}{|c|c|c|c|c|c|c|c|}
\hline & $\begin{array}{c}\mathrm{CD}^{+} \mathrm{T} \\
\text { cells }\end{array}$ & $\begin{array}{c}\mathrm{CD8}^{+} \mathrm{T} \\
\text { cells }\end{array}$ & B cells & NK cells & NK T cells & $\begin{array}{c}\text { Macropha } \\
\text { ges }\end{array}$ & DCs \\
\hline Naive & $\begin{array}{l}34 \pm 20 \\
\left(x 10^{5}\right)\end{array}$ & $\begin{array}{l}25 \pm 17 \\
\left(\times 10^{5}\right)\end{array}$ & $\begin{array}{l}15 \pm 9 \\
\left(\mathrm{x} 10^{5}\right)\end{array}$ & $\begin{array}{l}0.38 \pm 0.26 \\
\left(\mathrm{x} 10^{5}\right)\end{array}$ & $\begin{array}{l}0.17 \pm 0.15 \\
\left(\mathrm{x} 10^{5}\right)\end{array}$ & $\begin{array}{l}0.02 \pm 0.01 \\
\left(\times 10^{5}\right)\end{array}$ & $\begin{array}{l}1.7 \pm 1.4 \\
\left(\times 10^{5}\right)\end{array}$ \\
\hline E7+MPL & $\begin{array}{l}21 \pm 5 \\
\left(\times 10^{5}\right)\end{array}$ & $\begin{array}{l}17 \pm 5 \\
\left(\times 10^{5}\right)\end{array}$ & $\begin{array}{l}14 \pm 5 \\
\left(\mathrm{x} 10^{5}\right)\end{array}$ & $\begin{array}{l}0.19 \pm 0.03 \\
\left(\times 10^{5}\right)(*)\end{array}$ & $\begin{array}{l}0.15 \pm 0.12 \\
\left(\mathrm{x} 10^{5}\right)\end{array}$ & $\begin{array}{l}0.02 \pm 0.01 \\
\left(\times 10^{5}\right)(*)\end{array}$ & $2 \pm 1.2\left(\times 10^{5}\right)$ \\
\hline $\begin{array}{c}\text { E7+SA-4- } \\
\text { 1BBL }\end{array}$ & $\begin{array}{l}22 \pm 6 \\
\left(\times 10^{5}\right)\end{array}$ & $\begin{array}{l}18 \pm 3 \\
\left(\mathrm{x} 10^{5}\right)\end{array}$ & $\begin{array}{l}14 \pm 6 \\
\left(\mathrm{x} 10^{5}\right)\end{array}$ & $\begin{array}{l}0.29 \pm 0.13 \\
\left(\mathrm{x} 10^{5}\right)\end{array}$ & $\begin{array}{l}0.11 \pm 0.05 \\
\left(\times 10^{5}\right)\end{array}$ & $\begin{array}{l}0.03 \pm 0.02 \\
\left(\mathrm{x} 10^{5}\right)\end{array}$ & $\begin{array}{l}1.5 \pm 0.7 \\
\left(\times 10^{5}\right)\end{array}$ \\
\hline $\begin{array}{c}\text { E7+MPL+S } \\
\text { A-4-1BBL }\end{array}$ & $\begin{array}{l}24 \pm 8 \\
\left(\times 10^{5}\right)\end{array}$ & $\begin{array}{l}20 \pm 7 \\
\left(\times 10^{5}\right)\end{array}$ & $\begin{array}{l}17 \pm 7 \\
\left(\mathrm{x} 10^{5}\right)\end{array}$ & $\begin{array}{l}0.28 \pm 0.06 \\
\left(\mathrm{x} 10^{5}\right)\end{array}$ & $\begin{array}{l}0.13 \pm 0.04 \\
\left(\mathrm{x} 10^{5}\right)\end{array}$ & $\begin{array}{l}0.05 \pm 0.01 \\
\left(\times 10^{5}\right)\end{array}$ & $\begin{array}{l}1.7 \pm 0.6 \\
\left(\times 10^{5}\right)\end{array}$ \\
\hline
\end{tabular}




\section{Total numbers of lymphocytes in the mLN}

\begin{tabular}{|c|c|c|c|c|c|c|c|}
\hline & $\begin{array}{c}\mathbf{C D 4}^{+} \mathbf{T} \\
\text { cells }\end{array}$ & $\begin{array}{c}\mathbf{C D 8}^{+} \mathbf{T} \\
\text { cells }\end{array}$ & B cells & NK cells & NK T cells & $\begin{array}{l}\text { Macropha } \\
\text { ges }\end{array}$ & DCs \\
\hline Naive & $\begin{array}{c}69 \pm 24 \\
\left(\mathrm{x} 10^{5}\right)\end{array}$ & $\begin{array}{c}45 \pm 14 \\
\left(\mathrm{x} 10^{5}\right)\end{array}$ & $\begin{array}{l}50 \pm 19 \\
\left(\mathrm{x} 10^{5}\right)\end{array}$ & $\begin{array}{l}0.7 \pm 0.4 \\
\left(\mathrm{x} 10^{5}\right)\end{array}$ & $\begin{array}{l}0.4 \pm 0.2 \\
\left(\mathrm{x} 10^{5}\right)\end{array}$ & $\begin{array}{l}0.1 \pm 0.07 \\
\left(\mathrm{x} 10^{5}\right)\end{array}$ & $6 \pm 2\left(\mathrm{x} 10^{5}\right)$ \\
\hline E7+MPL & $\begin{array}{c}43 \pm 11 \\
\left(\mathrm{x} 10^{5}\right)\end{array}$ & $\begin{array}{c}32 \pm 8.3 \\
\left(\mathrm{x} 10^{5}\right)\end{array}$ & $\begin{array}{l}29 \pm 13 \\
\left(\mathrm{x} 10^{5}\right)\end{array}$ & $\begin{array}{l}0.3 \pm 0.1 \\
\left(\mathrm{x} 10^{5}\right)(*)\end{array}$ & $\begin{array}{l}0.3 \pm 0.07 \\
\left(\mathrm{x} 10^{5}\right)\end{array}$ & $\begin{array}{l}0.05 \pm 0.01 \\
\left(\mathrm{x} 10^{5}\right)\end{array}$ & $4 \pm 2\left(\mathrm{x} 10^{5}\right)$ \\
\hline $\begin{array}{c}\text { E7+SA-4- } \\
\text { 1BBL }\end{array}$ & $\begin{array}{c}55 \pm 29 \\
\left(\mathrm{x} 10^{5}\right)\end{array}$ & $\begin{array}{c}34 \pm 22 \\
\left(\mathrm{x} 10^{5}\right)\end{array}$ & $\begin{array}{l}49 \pm 16 \\
\left(\mathrm{x} 10^{5}\right)\end{array}$ & $\begin{array}{l}0.6 \pm 0.2 \\
\left(\mathrm{x} 10^{5}\right)\end{array}$ & $\begin{array}{l}0.3 \pm 0.1 \\
\left(\mathrm{x} 10^{5}\right)\end{array}$ & $\begin{array}{l}0.07 \pm 0.02 \\
\left(\mathrm{x} 10^{5}\right)\end{array}$ & $5 \pm 2\left(\mathrm{x} 10^{5}\right)$ \\
\hline $\begin{array}{c}\text { E7+MPL+S } \\
\text { A-4-1BBL }\end{array}$ & $\begin{array}{c}56 \pm 18 \\
\left(\mathrm{x} 10^{5}\right)\end{array}$ & $\begin{array}{c}38 \pm 15 \\
\left(\mathrm{x} 10^{5}\right)\end{array}$ & $\begin{array}{c}42 \pm 12 \\
\left(\mathrm{x} 10^{5}\right)\end{array}$ & $\begin{array}{l}0.5 \pm 0.1 \\
\left(\mathrm{x} 10^{5}\right)\end{array}$ & $\begin{array}{l}0.3 \pm 0.06 \\
\left(\mathrm{x} 10^{5}\right)\end{array}$ & $\begin{array}{l}0.07 \pm 0.02 \\
\left(\mathrm{x} 10^{5}\right)\end{array}$ & $5 \pm 1\left(\mathrm{x} 10^{5}\right)$ \\
& & & & & & & \\
\hline
\end{tabular}

\section{Table 1}

SA-4-1BBL + MPL adjuvant system does not induce acute lymphocyte recruitment into the lymphatic tissues. C57BL/6 mice ( $n=3$ per group) were vaccinated with indicated vaccine formulations or left untreated (PBS). $18 \mathrm{~h}$ after immunization, mice were terminated; spleen, dLNs, and mLN were harvested to determine the absolute numbers of indicated lymphocyte subsets. A) Absolute numbers of indicated lymphocytes in the spleen. ** $\mathrm{p} \leq 0.02$ in $\mathrm{E} 7+\mathrm{MPL} v s \mathrm{E} 7+\mathrm{MPL}+\mathrm{SA}-4-1 \mathrm{BBL}$; E7+MPL $v s \mathrm{E} 7+\mathrm{SA}-4-1 \mathrm{BBL}$; E7+MPL vs Naïve in NK cells. * $\mathrm{p} \leq 0.05$ in $\mathrm{E} 7+\mathrm{SA}-4-1 \mathrm{BBL}$ vs Naïve in macrophages. B) Absolute numbers of indicated lymphocytes in the dLNs. ${ }^{*} \mathrm{p} \leq 0.05$ in E7+MPL $v s$ E7+SA-4-1BBL+MPL in NK cells. ${ }^{*} \mathrm{p} \leq 0.05$ in E7+MPL $v s$ E7+SA-4-1BBL+MPL in macrophages. C) Absolute numbers of indicated lymphocytes in the $\mathrm{mLN}$. ${ }^{*} \mathrm{p} \leq 0.05$ in E7+MPL vs E7+SA-4-1BBL in NK cells. 

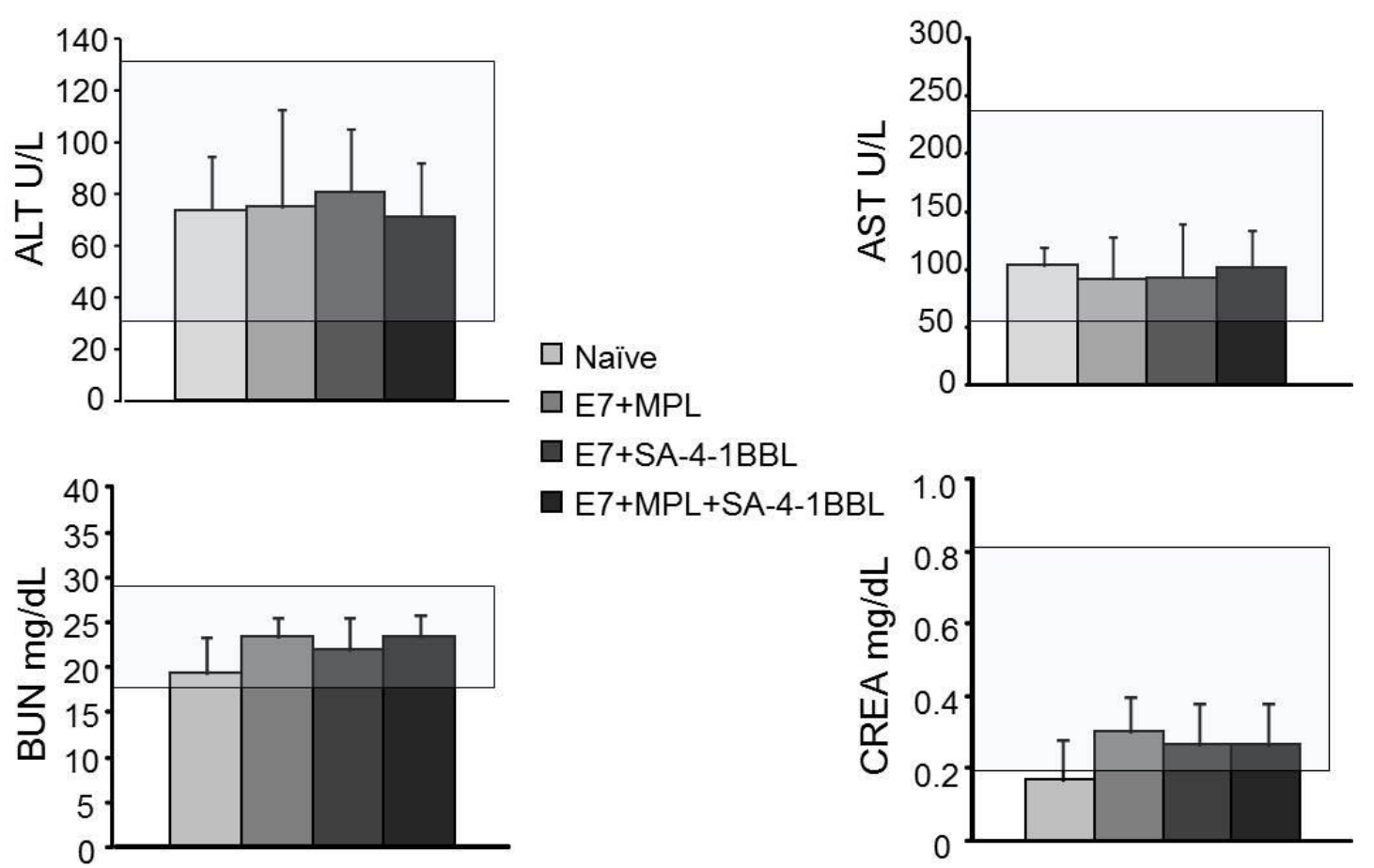

\section{Figure 18}

Treatment with SA-4-1BBL + MPL adjuvant system does not induce liver and kidney damage. C57BL/6 mice ( $\mathrm{n}=3$ per group) were vaccinated with indicated vaccine formulations or left untreated (PBS). $18 \mathrm{~h}$ after immunization, serum was collected to evaluate the kidney and liver enzyme levels. Rectangle area indicates the normal range for each enzyme level. ALT range: 28-132 U/L, AST range: 59-247 U/L, BUN range: 18$29 \mathrm{mg} / \mathrm{dL}$, CREA range: $0.2-0.8 \mathrm{mg} / \mathrm{dL}$. 


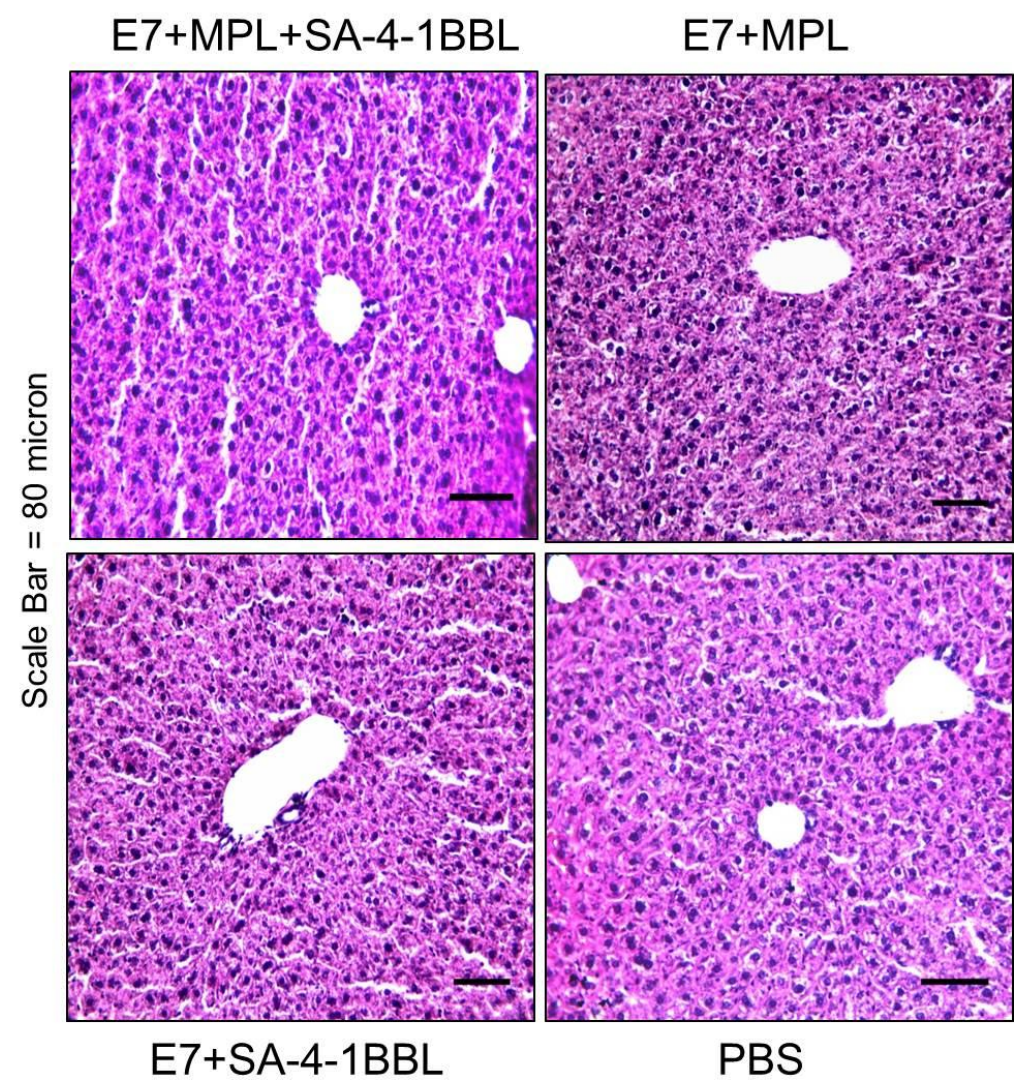

\section{Figure 19}

Treatment with SA-4-1BBL + MPL adjuvant system does not induce acute lymphocyte infiltration into the liver. C57BL/6 mice ( $\mathrm{n}=3$ per group) were vaccinated with indicated vaccine formulations or left untreated (PBS). 18h after immunization, livers were collected to determine the histo-pathology. 


\section{CHAPTER 4}

\section{CONCLUSIONS AND FUTURE PROSPECTIVES}

Prophylactic vaccines against infections have had a tremendous effect on individual health since it has significantly reduced the rate of deadly infectious diseases and human mortality. However, historically, vaccines were made from live-attenuated or inactivated forms of the microbe and there were concerns about stability, side effects and safety. As a result, with the development of molecular biology, different vaccine strategies have been investigated to generate new human vaccines. Vaccines based on recombinant proteins can overcome the limitations relating to the safety, and present an attractive approach because of their safety, ease of transportation, and storage. However, recombinant protein-based subunit vaccines are poorly immunogenic and need to be administered with adjuvants to generate an efficient appropriate immune response with long lasting immune memory against target antigens.

Historically, the importance of the humoral immune response for vaccine effectiveness has put the emphasis on the development of adjuvants, which are capable of enhancing antibody responses. As a result, many adjuvants are effective in increasing antibody titers, but do not elicit significant $\mathrm{Th}_{1}$ or CTL responses. However, for some viral and intracellular bacterial infections, generation of only antibody response is not 
enough for the complete protection. In this regard, combination of adjuvants with distinct action mechanisms should provide a great potential against such infections. For example, combination of TLR ligands that target primarily innate immunity and stimulate $\mathrm{Th}_{1}$ cytokine responses increases the efficacy of vaccines which primarily target the generation of antibody response (AS04 adjuvant system).

Plague is one of those infections that require both antibody and $\mathrm{Th}_{1}$ cellular responses. Therefore, in this study, we utilized SA-4-1BBL that targets primarily adaptive immunity as an adjuvant component of prophylactic bubonic plague vaccine either alone or in combination with alum that targets the innate immunity to induce the generation of cellular immune responses, and enhance the protective efficacy of the vaccine. Here, it has been shown that combination of SA-4-1BBL + alum induced a robust antigen specific $\mathrm{Th}_{1}$ cytokine response in $\mathrm{CD}^{+}$and $\mathrm{CD} 8^{+} \mathrm{T}$ cells, along with high anti-rF1-V titers which lead to $100 \%$ efficacy against bubonic plague in a prime-only setting, while SA-4-1BBL monotherapy resulted in $20 \%$ efficacy without generation of antibody response. However, administration of SA-4-1BBL monotherapy twice in a prime-boost setting induced robust and long-lasting antibody response and enhanced cellular response which translated into $100 \%$ efficacy. These results further support the requirement of a balanced cellular and humoral response in the case of plague infection, and future vaccines should be designed to target both humoral and cellular immunity with the utilization of combination adjuvants. 
Cancer, on the other hand, is a complex disease to overcome since tumor microenvironment can highly affect the disease progression and includes multiple cell types which interact with each other. Therefore, it affects millions of people and its incidence and human mortality is increasing all over the world. Although cancer immunotherapy has been chosen as breakthrough of the year for 2013 by Science's editors, as two strategies have been shown to be effective including anti-CTLA-4 antibody therapy, but the cost of these antibody therapies is too high, $\$ 120,000$, for a course therapy. On the other hand, the complexity of the preparation of individualized vaccines limits the use of whole tumor cell and dendritic cell-based vaccines. Therefore, development of recombinant sub-unit based vaccines might present an attractive approach because of their potential low cost, and ease of production.

Due to the low immunogenic nature of TAAs, adjuvants should be formulated in the subunit vaccines. However, not all the adjuvants are effective to generate anti-tumor immune responses with a therapeutic efficacy since the tumor-induced immunosuppressive mechanisms in the tumor microenvironment (TME) which are the biggest obstacles for therapeutic cancer vaccine development. The immunosuppressive mechanisms constitute expansion and/or recruitment of regulatory cells such as Tregs, MDSCs, and TAMs within the TME, and production and release of immune suppressive soluble factors such as TGF- $\beta$, IL-10, IDO via tumor cells. Therefore, immune modulators such as antibodies against CTLA-4, and PD-1 have been used to enhance the anti-tumor effector responses. However, such strategies are established to block or remove negative regulatory mechanisms, and may not be sufficient to induce potent antitumor efficacy due to the lack of the stimulation of antigen specific immune responses, 
and tumors are also capable of interfering innate and adaptive immune responses to evade from immune system attack. In this context, vaccines utilizing adjuvants which can modulate innate, adaptive and regulatory immunity will have better efficacy. Therefore, here we hypothesize that utilization of SA-4-1BBL as an adjuvant component of recombinant Her-2/neu subunit vaccine might improve the therapeutic efficacy of Her2/neu positive breast cancer vaccine due to its pleiotropic effects on the cells of innate, adaptive, and regulatory immunity either alone or in combination with MPL. Furthermore, its expression on the activated $\mathrm{CD}^{+} \mathrm{T}, \mathrm{CD} 8^{+} \mathrm{T}, \mathrm{NK}$ and $\mathrm{NK} \mathrm{T}$ cells provides another advantage to target antigen specific cells of innate and adaptive immunity. We show that combination of SA-4-1BBL + MPL generated robust tumor specific killing response, and inflammatory cytokine IFN- $\gamma$ production. The sum of these responses translated into better efficacy than the individual agents without detectable toxicity. As such, combined adjuvant therapy resulted in $30 \%$ eradication of established Her-2/neu breast cancer in BALB/c mice, while SA-4-1BBL monotherapy provided 10\% protection and MPL monotherapy failed to do so. Furthermore, combined adjuvant therapy eradicated the tumors in $20 \%$ of BALB/neuT mice, while depletion of Tregs further improved its efficacy to $40 \%$. These results further support the requirement of targeting both innate and adaptive immunity to generate potent immune response to eliminate the cancer cells and overcoming immune suppressive mechanisms employed by tumors.

On the other hand, although critical, augmented CTL responses may not translate into better anti-tumor efficacy since another obstacle is the trafficking of tumor-reactive CTLs into the tumor from the periphery and for the immune surveillance activated 
effector $\mathrm{T}$ cells must accumulate and function within the tumors. In this regard, SA-41BBL seems to be a potential adjuvant candidate since our lab has previously shown that SA-4-1BBL adjuvanted HPV E7-based therapeutic cervical cancer vaccine enhances the number of infiltrating $\mathrm{CD} 8^{+} \mathrm{T}$ cells to the tumor, and addition of MPL to the SA-4-1BBL therapy further increases the number of intratumoral $\mathrm{CD} 8^{+} \mathrm{T}$ cells infiltration. Moreover, a significant reduction in the number of intratumoral Tregs in mice vaccinated with either SA-4-1BBL as a single adjuvant or in combination with MPL has also been observed in that study. In this regard, chemokines such as CCL5, CXCL9, CXCL10, and Leukotriene B4 receptor BLT1 have been shown to regulate the selective CTL recruitment. Although it is not known whether SA-4-1BBL is up-regulating the expression of such chemokines, it is likely that it enhances the production of leukocyte chemoattractants due to the high $\mathrm{CD} 8^{+} \mathrm{T}$ cell numbers in the tumor.

There are three distinct steps that must be achieved to generate effective immunity against tumor or infection. First of all, DCs must be activated and present antigens derived from tumor, or pathogens. Activation signals could be generated with the recruitment of TLR ligands or agonistic antibodies against activating receptors such as CD40 in the vaccines. Next, antigen specific $\mathrm{T}$ cell activation must be induced in the lymphoid organs. In this context, the importance of co-stimulatory molecules for the activation of effector $\mathrm{T}$ lymphocytes has put the emphasis on the use of the agonists of co-stimulatory receptors as adjuvants in vaccines. Now, it has been realized that generation of the first signal through antigen presentation is not enough for $\mathrm{T}$ cell activation; rather this signal should be followed by signals from costimulatory molecules. Further, the quality of effector $\mathrm{CD} 4^{+}$and $\mathrm{CD} 8^{+} \mathrm{T}$ cells is more important than their high 
quantity. Finally, antigen-specific $\mathrm{T}$ cells must be recruited into the site of infection or tumor to perform their effector function where immune suppression takes place. Any vaccine approaches have to overcome such barriers to be successful. As a result, the failure of immunotherapy is very likely, and combining immunotherapies has shown to be efficacious and encourage scientists to investigate other combination approaches.

Although animal models show promising results for immunotherapy, human clinical trials have been somewhat disappointing because of the complexity and undefined differences between animals and humans. Despite the challenges to translate efficacy from animals to humans, vaccines against some infectious diseases and cancers have been developed and shown to be efficacious in humans with therapeutic efficacy, and some vaccines are already being tested in clinical trials and the ones which are effective and safe will be used in near future as well. However, there is still a dire need for developing vaccines against various acute and chronic infections and cancer.

It still remains challenging to develop effective vaccines against infectious diseases and cancer with recombinant proteins because of their poor immunogenic nature. Development of adjuvants to improve immune response to such vaccines has been increased dramatically in the last several years, and some adjuvants are currently employed in human vaccines. Targeting 4-1BB receptor presents an attractive approach due to its 1) pleiotropic effect on the innate, adaptive, and regulatory immunity, 2) expression on the activated but not resting $\mathrm{T}$ cells, and 3) $\mathrm{CD} 8^{+} \mathrm{T}$ cell centric function which is critical for the elimination of pathogen infected and tumor cells, and generation of immune memory. Moreover, significant progress has been made for the development of antibodies specific for 4-1-BB receptor for human use. 
The promising results from this overall Ph.D. study may provide a new scientific rationale for the utility of SA-4-1BBL costimulatory molecule as an adjuvant platform for the development of subunit vaccines either alone or in combination with other immune stimulators targeting primarily innate immunity against viral or bacterial infections requiring both humoral and cellular immune responses, and cancer. Use of immune stimulators targeting innate immunity provides enhanced APC activation and function which primes $\mathrm{T}$ cells, and use of SA-4-1BBL further increases $\mathrm{T}$ cell effector functions, survival, and memory responses.

Although treatment with SA-4-1BBL + MPL therapy resulted in eradication of transplanted tumors in $30 \%$ of $\mathrm{BALB} / \mathrm{c}$ mice and $20 \%$ of more tolerogenic $\mathrm{BALB} / \mathrm{neuT}$ mice, vaccine schedule and dosage should be further evaluated for a better therapeutic efficacy. After finding the most efficacious schedule and administration dosage, this potent combined adjuvant therapy should be tested for a spontaneous breast cancer model in BALB/neuT mice. Moreover, despite the $100 \%$ protection generated by SA-4-1BBL + alum in prime-only setting, and SA-4-1BBL adjuvanted vaccine in a prime-boost setting in the bubonic plague model, this potent adjuvant system should be tested in a more clinically relevant pneumonic plague model. To mimic the aerosol exposure to $Y$. pestis, and induce the immune response at mucosal surfaces, the vaccine could be administered intranasal. If it demonstrates efficacy in murine model, it should be tested in NHP models such as African green monkeys that have been difficult to confer protection. Although here we demonstrated effectiveness of the combined adjuvant systems in the clearance of intracellular bacteria and cancer, the mechanisms behind these therapies, and the synergistic effect should be further investigated for the clinical translation. SA-4- 
1BBL either alone or in combination with other adjuvants targeting different pathways should be tested in future pre-clinical models and clinical trials against plague and breast cancer. Eventually, testing these adjuvant systems in clinical trials will assess the therapeutic potential of these combined adjuvant platforms, and if efficacy is demonstrated, they can be utilized against other infection and cancer types. 


\section{REFERENCES}

1. $\quad$ Ertl, H.C. and Z. Xiang, Novel vaccine approaches. J Immunol, 1996. 156(10): p. 3579-82.

2. Plotkin, S.A., Of vaccination and infectious diseases in 2003. Adv Exp Med Biol, 2004. 549: p. 1-4.

3. Plotkin, S.A., Vaccines: past, present and future. Nat Med, 2005. 11(4 Suppl): p. S5-11.

4. Reed, S.G., M.T. Orr, and C.B. Fox, Key roles of adjuvants in modern vaccines. Nat Med, 2013. 19(12): p. 1597-608.

5. Nascimento, I.P. and L.C. Leite, Recombinant vaccines and the development of new vaccine strategies. Braz J Med Biol Res, 2012. 45(12): p. 1102-11.

6. Didierlaurent, A.M., et al., ASO4, an aluminum salt- and TLR4 agonist-based adjuvant system, induces a transient localized innate immune response leading to enhanced adaptive immunity. J Immunol, 2009. 183(10): p. 6186-97.

7. Pulendran, B. and R. Ahmed, Translating innate immunity into immunological memory: implications for vaccine development. Cell, 2006. 124(4): p. 849-63.

8. Curtsinger, J.M., et al., Inflammatory cytokines provide a third signal for activation of naive CD4+ and CD8+ T cells. J Immunol, 1999. 162(6): p. 325662.

9. Tscharke, D.C. and A. Suhrbier, From mice to humans - murine intelligence for human CD8+ T cell vaccine design. Expert Opin Biol Ther, 2005. 5(2): p. 26371.

10. Perrie, Y., et al., Vaccine adjuvant systems: enhancing the efficacy of sub-unit protein antigens. Int J Pharm, 2008. 364(2): p. 272-80.

11. O'Hagan, D.T., M.L. MacKichan, and M. Singh, Recent developments in adjuvants for vaccines against infectious diseases. Biomol Eng, 2001. 18(3): p. 69-85.

12. McElrath, M.J., Selection of potent immunological adjuvants for vaccine construction. Semin Cancer Biol, 1995. 6(6): p. 375-85.

13. Awate, S., L.A. Babiuk, and G. Mutwiri, Mechanisms of action of adjuvants. Front Immunol, 2013. 4: p. 114.

14. Cox, J.C. and A.R. Coulter, Adjuvants--a classification and review of their modes of action. Vaccine, 1997. 15(3): p. 248-56.

15. Pashine, A., N.M. Valiante, and J.B. Ulmer, Targeting the innate immune response with improved vaccine adjuvants. Nat Med, 2005. 11(4 Suppl): p. S63-8.

16. Disis, M.L., et al., Existent T-cell and antibody immunity to HER-2/neu protein in patients with breast cancer. Cancer Res, 1994. 54(1): p. 16-20.

17. Disis, M.L., et al., High-titer HER-2/neu protein-specific antibody can be detected in patients with early-stage breast cancer. J Clin Oncol, 1997. 15(11): p. 3363-7. 
18. Barouch, D.H., N.L. Letvin, and R.A. Seder, The role of cytokine DNAs as vaccine adjuvants for optimizing cellular immune responses. Immunol Rev, 2004. 202: p. 266-74.

19. Kasturi, S.P., et al., Programming the magnitude and persistence of antibody responses with innate immunity. Nature, 2011. 470(7335): p. 543-7.

20. Khurana, S., et al., MF59 adjuvant enhances diversity and affinity of antibodymediated immune response to pandemic influenza vaccines. Sci Transl Med, 2011. 3(85): p. 85ra48.

21. Draper, E., et al., A randomized, observer-blinded immunogenicity trial of Cervarix $((R))$ and Gardasil( $(R))$ Human Papillomavirus vaccines in 12-15 year old girls. PLoS One, 2013. 8(5): p. e61825.

22. Kensil, C.R., Saponins as vaccine adjuvants. Crit Rev Ther Drug Carrier Syst, 1996. 13(1-2): p. 1-55.

23. Takahashi, H., et al., Induction of CD8+ cytotoxic T cells by immunization with purified HIV-1 envelope protein in ISCOMs. Nature, 1990. 344(6269): p. 873-5.

24. Kensil, C.R. and R. Kammer, QS-21: a water-soluble triterpene glycoside adjuvant. Expert Opin Investig Drugs, 1998. 7(9): p. 1475-82.

25. Cox, M. Update on clinical trials evaluation of adjuvanted $\mathrm{rHA}(\mathrm{H} 5)$ vaccines. in 7th WHO Meeting on Evaluation of Pandemic Influenza Vaccines in Clinical Trials (Geneva, Switzerland, 2011)

26. Website Goodman JL.Workshop: Adjuvants and Adjuvanted Preventive and Therapeutic Vaccines for Infectious Disease Indications; Bethesda, MD, USA. December 2 \& 3, 2008;

http://www.fda.gov/downloads/BiologicsBloodVaccines/NewsEvents/Workshops MeetingsConferences/UCM095732.pdf

27. Duthie, M.S., et al., Use of defined TLR ligands as adjuvants within human vaccines. Immunol Rev, 2011. 239(1): p. 178-96.

28. Carter, D. and S.G. Reed, Role of adjuvants in modeling the immune response. Curr Opin HIV AIDS, 2010. 5(5): p. 409-13.

29. Petrovsky, N. and J.C. Aguilar, Vaccine adjuvants: current state and future trends. Immunol Cell Biol, 2004. 82(5): p. 488-96.

30. Lindblad, E.B., Aluminium adjuvants--in retrospect and prospect. Vaccine, 2004. 22(27-28): p. 3658-68.

31. Burdin, N., B. Guy, and P. Moingeon, Immunological foundations to the quest for new vaccine adjuvants. BioDrugs, 2004. 18(2): p. 79-93.

32. Garcon, N., P. Chomez, and M. Van Mechelen, GlaxoSmithKline Adjuvant Systems in vaccines: concepts, achievements and perspectives. Expert Rev Vaccines, 2007. 6(5): p. 723-39.

33. White, R.G., A.H. Coons, and J.M. Connolly, Studies on antibody production. III. The alum granuloma. J Exp Med, 1955. 102(1): p. 73-82.

34. Gupta, R.K., et al., Adjuvant properties of aluminum and calcium compounds. Pharm Biotechnol, 1995. 6: p. 229-48.

35. Podda, A. and G. Del Giudice, MF59-adjuvanted vaccines: increased immunogenicity with an optimal safety profile. Expert Rev Vaccines, 2003. 2(2): p. 197-203. 
36. Novartis receives US government contract to further develop a novel antigen technology that could extend vaccine supplies in a pandemic outbreak. 2007. (Accessed January 30, 2007, at http://novartisvaccines.com/pressroom/news/20070117.shtml.)

37. Mosca, F., et al., Molecular and cellular signatures of human vaccine adjuvants. Proc Natl Acad Sci U S A, 2008. 105(30): p. 10501-6.

38. Dupuis, M., et al., Dendritic cells internalize vaccine adjuvant after intramuscular injection. Cell Immunol, 1998. 186(1): p. 18-27.

39. Wack, A., et al., Combination adjuvants for the induction of potent, long-lasting antibody and T-cell responses to influenza vaccine in mice. Vaccine, 2008. 26(4): p. 552-61.

40. Satoh, M., et al., Induction of lupus autoantibodies by adjuvants. J Autoimmun, 2003. 21(1): p. 1-9.

41. Rajput, Z.I., et al., Adjuvant effects of saponins on animal immune responses. J Zhejiang Univ Sci B, 2007. 8(3): p. 153-61.

42. Sun, H.X., Y. Xie, and Y.P. Ye, Advances in saponin-based adjuvants. Vaccine, 2009. 27(12): p. 1787-96.

43. Cox, J.C., A. Sjolander, and I.G. Barr, ISCOMs and other saponin based adjuvants. Adv Drug Deliv Rev, 1998. 32(3): p. 247-271.

44. Sjolander, A., et al., Immune responses to ISCOM formulations in animal and primate models. Vaccine, 2001. 19(17-19): p. 2661-5.

45. Soltysik, S., et al., Structurelfunction studies of QS-21 adjuvant: assessment of triterpene aldehyde and glucuronic acid roles in adjuvant function. Vaccine, 1995. 13(15): p. 1403-10.

46. Helling, F., et al., GM2-KLH conjugate vaccine: increased immunogenicity in melanoma patients after administration with immunological adjuvant QS-21. Cancer Res, 1995. 55(13): p. 2783-8.

47. Oda, K., et al., Adjuvant and haemolytic activities of 47 saponins derived from medicinal and food plants. Biol Chem, 2000. 381(1): p. 67-74.

48. Fehniger, T.A., M.A. Cooper, and M.A. Caligiuri, Interleukin-2 and interleukin15: immunotherapy for cancer. Cytokine Growth Factor Rev, 2002. 13(2): p. 16983.

49. Good, M.F., et al., Recombinant human IL-2 overcomes genetic nonresponsiveness to malaria sporozoite peptides. Correlation of effect with biologic activity of IL-2. J Immunol, 1988. 141(3): p. 972-7.

50. Rosenberg, S.A., et al., Immunologic and therapeutic evaluation of a synthetic peptide vaccine for the treatment of patients with metastatic melanoma. Nat Med, 1998. 4(3): p. 321-7.

51. Hersey, P., et al., Phase I/II study of treatment with matured dendritic cells with or without low dose IL-2 in patients with disseminated melanoma. Cancer Immunol Immunother, 2008. 57(7): p. 1039-51.

52. Lotem, M., et al., Interleukin-2 improves tumour response to DNP-modified autologous vaccine for the treatment of metastatic malignant melanoma. $\mathrm{Br} \mathrm{J}$ Cancer, 2004. 90(4): p. 773-80. 
53. Wei, Y., et al., Dendritoma vaccination combined with low dose interleukin-2 in metastatic melanoma patients induced immunological and clinical responses. Int J Oncol, 2006. 28(3): p. 585-93.

54. Rosenberg, S.A., et al., Recombinant fowlpox viruses encoding the anchormodified gp100 melanoma antigen can generate antitumor immune responses in patients with metastatic melanoma. Clin Cancer Res, 2003. 9(8): p. 2973-80.

55. Smith, F.O., et al., Treatment of metastatic melanoma using interleukin-2 alone or in conjunction with vaccines. Clin Cancer Res, 2008. 14(17): p. 5610-8.

56. Waldmann, T.A., The biology of interleukin-2 and interleukin-15: implications for cancer therapy and vaccine design. Nat Rev Immunol, 2006. 6(8): p. 595-601.

57. Zhang, H., et al., Lymphopenia and interleukin-2 therapy alter homeostasis of CD4+CD25+ regulatory T cells. Nat Med, 2005. 11(11): p. 1238-43.

58. Capitini, C.M., T.J. Fry, and C.L. Mackall, Cytokines as Adjuvants for Vaccine and Cellular Therapies for Cancer. Am J Immunol, 2009. 5(3): p. 65-83.

59. Rosenberg, S.A., et al., IL-7 administration to humans leads to expansion of $C D 8+$ and $C D 4+$ cells but a relative decrease of CD4+ T-regulatory cells. $\mathrm{J}$ Immunother, 2006. 29(3): p. 313-9.

60. Sportes, C., et al., Administration of rhIL-7 in humans increases in vivo TCR repertoire diversity by preferential expansion of naive T cell subsets. J Exp Med, 2008. 205(7): p. 1701-14.

61. Thompson, J.A., et al., Phase I study of recombinant interleukin-21 in patients with metastatic melanoma and renal cell carcinoma. J Clin Oncol, 2008. 26(12): p. 2034-9.

62. Scheeren, F.A., et al., IL-21 is expressed in Hodgkin lymphoma and activates STAT5: evidence that activated STAT5 is required for Hodgkin lymphomagenesis. Blood, 2008. 111(9): p. 4706-15.

63. Menoret, E., et al., IL-21 stimulates human myeloma cell growth through an autocrine IGF-1 loop. J Immunol, 2008. 181(10): p. 6837-42.

64. Meyer, T., et al., Resiquimod, a topical drug for viral skin lesions and skin cancer. Expert Opin Investig Drugs, 2013. 22(1): p. 149-59.

65. Barry, M. and C. Cooper, Review of hepatitis B surface antigen-1018 ISS adjuvant-containing vaccine safety and efficacy. Expert Opin Biol Ther, 2007. 7(11): p. 1731-7.

66. Schijns, V.E., Mechanisms of vaccine adjuvant activity: initiation and regulation of immune responses by vaccine adjuvants. Vaccine, 2003. 21(9-10): p. 829-31.

67. Kawai, T. and S. Akira, The role of pattern-recognition receptors in innate immunity: update on Toll-like receptors. Nat Immunol, 2010. 11(5): p. 373-84.

68. Gupta, R.K., et al., Adjuvants--a balance between toxicity and adjuvanticity. Vaccine, 1993. 11(3): p. 293-306.

69. Chen, L. and D.B. Flies, Molecular mechanisms of T cell co-stimulation and coinhibition. Nat Rev Immunol, 2013. 13(4): p. 227-42.

70. Croft, M., The role of TNF superfamily members in T-cell function and diseases. Nat Rev Immunol, 2009. 9(4): p. 271-85.

71. Berzofsky, J.A., M. Terabe, and L.V. Wood, Strategies to use immune modulators in therapeutic vaccines against cancer. Semin Oncol, 2012. 39(3): p. 348-57. 
72. Curran, M.A., et al., Combination CTLA-4 blockade and 4-1BB activation enhances tumor rejection by increasing $T$-cell infiltration, proliferation, and cytokine production. PLoS One, 2011. 6(4): p. e19499.

73. Takeda, K., et al., Combination therapy of established tumors by antibodies targeting immune activating and suppressing molecules. J Immunol, 2010.

184(10): p. 5493-501.

74. Townsend, S.E. and J.P. Allison, Tumor rejection after direct costimulation of CD8+ T cells by B7-transfected melanoma cells. Science, 1993. 259(5093): p. 368-70.

75. $\mathrm{Yu}, \mathrm{X} ., \mathrm{R} . \mathrm{Abe}$, and R.J. Hodes, The role of B7-CD28 co-stimulation in tumor rejection. Int Immunol, 1998. 10(6): p. 791-7.

76. Boise, L.H., et al., CD28 costimulation can promote T cell survival by enhancing the expression of Bcl-XL. Immunity, 1995. 3(1): p. 87-98.

77. Bluestone, J.A., E.W. St Clair, and L.A. Turka, CTLA4Ig: bridging the basic immunology with clinical application. Immunity, 2006. 24(3): p. 233-8.

78. Snanoudj, R., et al., The blockade of T-cell co-stimulation as a therapeutic stratagem for immunosuppression: Focus on belatacept. Biologics, 2007. 1(3): p. 203-13.

79. Sayers, T.J., et al., Antitumor effects of alpha-interferon and gamma-interferon on a murine renal cancer (Renca) in vitro and in vivo. Cancer Res, 1990. 50(17): p. 5414-20.

80. Wigginton, J.M., et al., Administration of interleukin 12 with pulse interleukin 2 and the rapid and complete eradication of murine renal carcinoma. J Natl Cancer Inst, 1996. 88(1): p. 38-43.

81. Croft, M., C.A. Benedict, and C.F. Ware, Clinical targeting of the TNF and TNFR superfamilies. Nat Rev Drug Discov, 2013. 12(2): p. 147-68.

82. Ascierto, P.A., et al., Clinical experiences with anti-CD137 and anti-PD1 therapeutic antibodies. Semin Oncol, 2010. 37(5): p. 508-16.

83. Byrd, J.C., et al., Phase I study of the anti-CD40 humanized monoclonal antibody lucatumumab (HCD122) in relapsed chronic lymphocytic leukemia. Leuk Lymphoma, 2012. 53(11): p. 2136-42.

84. Mbow, M.L., et al., New adjuvants for human vaccines. Curr Opin Immunol, 2010. 22(3): p. 411-6.

85. Garcon, N. and M. Van Mechelen, Recent clinical experience with vaccines using $M P L$ - and QS-21-containing adjuvant systems. Expert Rev Vaccines, 2011. 10(4): p. 471-86.

86. Garcon, N., D.W. Vaughn, and A.M. Didierlaurent, Development and evaluation of ASO3, an Adjuvant System containing alpha-tocopherol and squalene in an oilin-water emulsion. Expert Rev Vaccines, 2012. 11(3): p. 349-66.

87. Govan, V.A., A novel vaccine for cervical cancer: quadrivalent human papillomavirus (types 6, 11, 16 and 18) recombinant vaccine (Gardasil). Ther Clin Risk Manag, 2008. 4(1): p. 65-70.

88. Bayry, J., et al., In silico identified CCR4 antagonists target regulatory $T$ cells and exert adjuvant activity in vaccination. Proc Natl Acad Sci U S A, 2008. 105(29): p. 10221-6. 
89. Davies, M.N., et al., Toward the discovery of vaccine adjuvants: coupling in silico screening and in vitro analysis of antagonist binding to human and mouse CCR4 receptors. PLoS One, 2009. 4(11): p. e8084.

90. Bukczynski, J., et al., Costimulatory ligand 4-1BBL (CD137L) as an efficient adjuvant for human antiviral cytotoxic T cell responses. Proc Natl Acad Sci U S A, 2004. 101(5): p. 1291-6.

91. Futagawa, T., et al., Expression and function of 4-1BB and 4-1BB ligand on murine dendritic cells. Int Immunol, 2002. 14(3): p. 275-86.

92. Lee, H.W., et al., 4-1BB enhances CD8+ T cell expansion by regulating cell cycle progression through changes in expression of cyclins $D$ and $E$ and cyclindependent kinase inhibitor p27kip1. Eur J Immunol, 2003. 33(8): p. 2133-41.

93. Choi, B.K., et al., 4-1BB-dependent inhibition of immunosuppression by activated CD4+CD25+ T cells. J Leukoc Biol, 2004. 75(5): p. 785-91.

94. Robertson, S.J., et al., CD137 costimulation of CD8+ T cells confers resistance to suppression by virus-induced regulatory T cells. J Immunol, 2008. 180(8): p. 5267-74.

95. Krishnadas, D.K., et al., Wilms' tumor 1-specific cytotoxic T lymphocytes can be expanded from adult donors and cord blood. Leuk Res, 2011. 35(11): p. 1520-6.

96. Myers, L., et al., Combined CD137 (4-1BB) and adjuvant therapy generates a developing pool of peptide-specific CD8 memory T cells. Int Immunol, 2006. 18(2): p. 325-33.

97. Lin, G.H., et al., Endogenous 4-1BB ligand plays a critical role in protection from influenza-induced disease. J Immunol, 2009. 182(2): p. 934-47.

98. Sharma, R.K., et al., Costimulation as a platform for the development of vaccines: a peptide-based vaccine containing a novel form of 4-1BB ligand eradicates established tumors. Cancer Res, 2009. 69(10): p. 4319-26.

99. Lynch, D.H., The promise of 4-1BB (CD137)-mediated immunomodulation and the immunotherapy of cancer. Immunol Rev, 2008. 222: p. 277-86.

100. Rabu, C., et al., Production of recombinant human trimeric CD137L (4-1BBL). Cross-linking is essential to its T cell co-stimulation activity. J Biol Chem, 2005. 280(50): p. 41472-81.

101. Elpek, K.G., et al., Ex vivo expansion of $C D 4+C D 25+F o x P 3+T$ regulatory cells based on synergy between IL-2 and 4-1BB signaling. J Immunol, 2007. 179(11): p. 7295-304.

102. Sharma, R.K., et al., 4-1BB ligand as an effective multifunctional immunomodulator and antigen delivery vehicle for the development of therapeutic cancer vaccines. Cancer Res, 2010. 70(10): p. 3945-54.

103. Schabowsky, R.H., et al., A novel form of 4-1BBL has better immunomodulatory activity than an agonistic anti-4-1BB Ab without Ab-associated severe toxicity. Vaccine, 2009. 28(2): p. 512-22.

104. Sharma, R.K., et al., SA-4-1BBL as the immunomodulatory component of a HPV16 E7 protein based vaccine shows robust therapeutic efficacy in a mouse cervical cancer model. Vaccine, 2010. 28(36): p. 5794-802.

105. Sharma, R.K., et al., CD4(+) T Cells Play a Critical Role in the Generation of Primary and Memory Antitumor Immune Responses Elicited by SA-4-1BBL and TAA-Based Vaccines in Mouse Tumor Models. PLoS One, 2013. 8(9): p. e73145. 
106. Madireddi, S., et al., SA-4-1BBL costimulation inhibits conversion of conventional CD4+ T cells into CD4+ FoxP3 + T regulatory cells by production of IFNgamma. PLoS One, 2012. 7(8): p. e42459.

107. Srivastava, A.K., et al., Prime-boost vaccination with SA-4-1BBL costimulatory molecule and survivin eradicates lung carcinoma in $\mathrm{CD} 8+\mathrm{T}$ and $\mathrm{NK}$ cell dependent manner. PLoS One, 2012. 7(11): p. e48463.

108. Perry, R.D. and J.D. Fetherston, Yersinia pestis--etiologic agent of plague. Clin Microbiol Rev, 1997. 10(1): p. 35-66.

109. Sun, W., K.L. Roland, and R. Curtiss, 3rd, Developing live vaccines against plague. J Infect Dev Ctries, 2011. 5(9): p. 614-27.

110. Christian, M.D., Biowarfare and bioterrorism. Crit Care Clin, 2013. 29(3): p. 717-56.

111. Kool, J.L., Risk of person-to-person transmission of pneumonic plague. Clin Infect Dis, 2005. 40(8): p. 1166-72.

112. Galimand, M., et al., Multidrug resistance in Yersinia pestis mediated by a transferable plasmid. N Engl J Med, 1997. 337(10): p. 677-80.

113. Williamson, E.D., et al., A new improved sub-unit vaccine for plague: the basis of protection. FEMS Immunol Med Microbiol, 1995. 12(3-4): p. 223-30.

114. Williamson, E.D. and P.C. Oyston, Protecting against plague: towards a nextgeneration vaccine. Clin Exp Immunol, 2013. 172(1): p. 1-8.

115. Anderson, G.W., Jr., et al., Short- and long-term efficacy of single-dose subunit vaccines against Yersinia pestis in mice. Am J Trop Med Hyg, 1998. 58(6): p. 793-9.

116. Williamson, E.D., et al., Local and systemic immune response to a microencapsulated sub-unit vaccine for plague. Vaccine, 1996. 14(17-18): p. 1613-9.

117. Heath, D.G., et al., Protection against experimental bubonic and pneumonic plague by a recombinant capsular F1-V antigen fusion protein vaccine. Vaccine, 1998. 16(11-12): p. 1131-7.

118. Jones, S.M., et al., Protective efficacy of a fully recombinant plague vaccine in the guinea pig. Vaccine, 2003. 21(25-26): p. 3912-8.

119. WebsitePitt ML. Non-human primates as a model for pneumonic plague; Animals Models and Correlates of Protection for Plague Vaccines Workshop; Gaithersburg, MD, USA. 13-14 October 2004; www.fda.gov/cber/minutes/ plague101304t.pdf.

120. Smiley, S.T., Current challenges in the development of vaccines for pneumonic plague. Expert Rev Vaccines, 2008. 7(2): p. 209-21.

121. Green, M., et al., The SCID/Beige mouse as a model to investigate protection against Yersinia pestis. FEMS Immunol Med Microbiol, 1999. 23(2): p. 107-13.

122. Williamson, E.D., et al., A sub-unit vaccine elicits IgG in serum, spleen cell cultures and bronchial washings and protects immunized animals against pneumonic plague. Vaccine, 1997. 15(10): p. 1079-84.

123. Anderson, G.W., Jr., et al., Protection of mice from fatal bubonic and pneumonic plague by passive immunization with monoclonal antibodies against the F1 protein of Yersinia pestis. Am J Trop Med Hyg, 1997. 56(4): p. 471-3. 
124. Fellows, P., et al., Protection in mice passively immunized with serum from cynomolgus macaques and humans vaccinated with recombinant plague vaccine (rF1V). Vaccine, 2010. 28(49): p. 7748-56.

125. Bashaw, J., et al., Development of in vitro correlate assays of immunity to infection with Yersinia pestis. Clin Vaccine Immunol, 2007. 14(5): p. 605-16.

126. WebsitePitt ML. Non-human primates as a model for pneumonic plague; Animals Models and Correlates of Protection for Plague Vaccines Workshop; Gaithersburg, MD, USA. 13-14 October 2004; www.fda.gov/cber/minutes/ plague101304t.pdf.

127. African green monkeys generated much lower titers against F1 (though consistent), demonstrated high variability in anti-LcrV Ab titers, and did not show a correlation between $\mathrm{Ab}$ titers and protection

128. WebsitePitt ML. Non-human primates as a model for pneumonic plague; Animals Models and Correlates of Protection for Plague Vaccines Workshop; Gaithersburg, MD, USA. 13-14 October 2004; www.fda.gov/cber/minutes/ plague101304t.pdf.

129. Parent, M.A., et al., Gamma interferon, tumor necrosis factor alpha, and nitric oxide synthase 2, key elements of cellular immunity, perform critical protective functions during humoral defense against lethal pulmonary Yersinia pestis infection. Infect Immun, 2006. 74(6): p. 3381-6.

130. Kummer, L.W., et al., Antibodies and cytokines independently protect against pneumonic plague. Vaccine, 2008. 26(52): p. 6901-7.

131. Lin, J.S., et al., TNFalpha and IFNgamma contribute to F1/LcrV-targeted immune defense in mouse models of fully virulent pneumonic plague. Vaccine, 2010. 29(2): p. 357-62.

132. Nakajima, R. and R.R. Brubaker, Association between virulence of Yersinia pestis and suppression of gamma interferon and tumor necrosis factor alpha. Infect Immun, 1993. 61(1): p. 23-31.

133. Parent, M.A., et al., Cell-mediated protection against pulmonary Yersinia pestis infection. Infect Immun, 2005. 73(11): p. 7304-10.

134. Kilinc, M.O., et al., Generation of a multimeric form of CD4OL with potent immunostimulatory activity using streptavidin as a chaperon. Exp Mol Pathol, 2006. 80(3): p. 252-61.

135. Yolcu, E.S., et al., Cell membrane modification for rapid display of proteins as a novel means of immunomodulation: FasL-decorated cells prevent islet graft rejection. Immunity, 2002. 17(6): p. 795-808.

136. Schabowsky, R.H., et al., ProtEx technology for the generation of novel therapeutic cancer vaccines. Exp Mol Pathol, 2009. 86(3): p. 198-207.

137. Sun, Y., et al., Development of bioluminescent bioreporters for in vitro and in vivo tracking of Yersinia pestis. PLoS One, 2012. 7(10): p. e47123.

138. Warawa, J.M. and M.B. Lawrenz, Bioluminescent imaging of bacteria during mouse infection. Methods Mol Biol, 2014. 1098: p. 169-81.

139. Brewer, J.M., et al., Aluminium hydroxide adjuvant initiates strong antigenspecific Th2 responses in the absence of IL-4- or IL-13-mediated signaling. J Immunol, 1999. 163(12): p. 6448-54. 
140. Uddowla, S., L.C. Freytag, and J.D. Clements, Effect of adjuvants and route of immunizations on the immune response to recombinant plague antigens. Vaccine, 2007. 25(47): p. 7984-93.

141. Pollock, K.G., et al., Interleukin-18 plays a role in both the alum-induced $T$ helper 2 response and the Thelper 1 response induced by alum-adsorbed interleukin-12. Immunology, 2003. 108(2): p. 137-43.

142. Aimanianda, V., et al., Novel cellular and molecular mechanisms of induction of immune responses by aluminum adjuvants. Trends Pharmacol Sci, 2009. 30(6): p. 287-95.

143. Jankovic, D., et al., Adsorption to aluminum hydroxide promotes the activity of IL-12 as an adjuvant for antibody as well as type 1 cytokine responses to HIV-I gp120. J Immunol, 1997. 159(5): p. 2409-17.

144. Martin, R.M., J.L. Brady, and A.M. Lew, The need for IgG2c specific antiserum when isotyping antibodies from C57BL/6 and NOD mice. J Immunol Methods, 1998. 212(2): p. 187-92.

145. Hill, J., et al., Synergistic protection of mice against plague with monoclonal antibodies specific for the F1 and V antigens of Yersinia pestis. Infect Immun, 2003. 71(4): p. 2234-8.

146. Williamson, E.D. and P.C. Oyston, The natural history and incidence of Yersinia pestis and prospects for vaccination. J Med Microbiol, 2012. 61(Pt 7): p. 911-8.

147. WebsitePitt ML. Non-human primates as a model for pneumonic plague; Animals Models and Correlates of Protection for Plague Vaccines Workshop; Gaithersburg, MD, USA. 13-14 October 2004; www.fda.gov/cber/minutes/ plague101304t.pdf.

148. Seder, R.A. and A.V. Hill, Vaccines against intracellular infections requiring cellular immunity. Nature, 2000. 406(6797): p. 793-8.

149. Su, Z., et al., Vaccination with novel immunostimulatory adjuvants against bloodstage malaria in mice. Infect Immun, 2003. 71(9): p. 5178-87.

150. Choi, B.K., et al., 4-1BB functions as a survival factor in dendritic cells. J Immunol, 2009. 182(7): p. 4107-15.

151. Kuang, Y., et al., Effects of 4-1BB signaling on the biological function of murine dendritic cells. Oncol Lett, 2012. 3(2): p. 477-481.

152. Kim, Y.J. and H.E. Broxmeyer, Therapeutic potential of 4-1BB (CD137) as a regulator for effector CD8(+) T cells. J Hematother Stem Cell Res, 2001. 10(4): p. 441-9.

153. Kim, Y.J., et al., Human 4-1BB regulates CD28 co-stimulation to promote Th1 cell responses. Eur J Immunol, 1998. 28(3): p. 881-90.

154. Hurtado, J.C., et al., Potential role of 4-1BB in T cell activation. Comparison with the costimulatory molecule CD28. J Immunol, 1995. 155(7): p. 3360-7.

155. Acquavella, N., et al., Toxicity and activity of a twice daily high-dose bolus interleukin 2 regimen in patients with metastatic melanoma and metastatic renal cell cancer. J Immunother, 2008. 31(6): p. 569-76.

156. Bekaii-Saab, T.S., et al., A phase I trial of paclitaxel and trastuzumab in combination with interleukin-12 in patients with HER2/neu-expressing malignancies. Mol Cancer Ther, 2009. 8(11): p. 2983-91. 
157. Karbach, J., et al., Therapeutic administration of a synthetic $C p G$ oligodeoxynucleotide triggers formation of anti-CpG antibodies. Cancer Res, 2012. 72(17): p. 4304-10.

158. Wang, S., et al., Involvement of CD8+T cell-mediated immune responses in LcrV DNA vaccine induced protection against lethal Yersinia pestis challenge.

Vaccine, 2011. 29(39): p. 6802-9.

159. Takahashi, C., R.S. Mittler, and A.T. Vella, Cutting edge: 4-1BB is a bona fide CD8 T cell survival signal. J Immunol, 1999. 162(9): p. 5037-40.

160. Shuford, W.W., et al., 4-1BB costimulatory signals preferentially induce CD8+T cell proliferation and lead to the amplification in vivo of cytotoxic $T$ cell responses. J Exp Med, 1997. 186(1): p. 47-55.

161. Philipovskiy, A.V. and S.T. Smiley, Vaccination with live Yersinia pestis primes CD4 and CD8 T cells that synergistically protect against lethal pulmonary $Y$. pestis infection. Infect Immun, 2007. 75(2): p. 878-85.

162. Lukaszewski, R.A., et al., Pathogenesis of Yersinia pestis infection in BALB/C mice: effects on host macrophages and neutrophils. Infect Immun, 2005. 73(11): p. 7142-50.

163. Lefeber, D.J., et al., Th1-directing adjuvants increase the immunogenicity of oligosaccharide-protein conjugate vaccines related to Streptococcus pneumoniae type 3. Infect Immun, 2003. 71(12): p. 6915-20.

164. Li, B. and R. Yang, Interaction between Yersinia pestis and the host immune system. Infect Immun, 2008. 76(5): p. 1804-11.

165. Weeks, S., et al., Anti-V antigen antibody protects macrophages from Yersinia pestis -induced cell death and promotes phagocytosis. Microb Pathog, 2002. 32(5): p. 227-37.

166. Cowan, C., et al., Anti-LcrV antibody inhibits delivery of Yops by Yersinia pestis KIM5 by directly promoting phagocytosis. Infect Immun, 2005. 73(9): p. 6127-37.

167. Snapper, C.M. and W.E. Paul, Interferon-gamma and B cell stimulatory factor-1 reciprocally regulate Ig isotype production. Science, 1987. 236(4804): p. 944-7.

168. Tohyama, Y. and H. Yamamura, Complement-mediated phagocytosis--the role of Syk. IUBMB Life, 2006. 58(5-6): p. 304-8.

169. WebsitePitt ML. Non-human primates as a model for pneumonic plague; Animals Models and Correlates of Protection for Plague Vaccines Workshop; Gaithersburg, MD, USA. 13-14 October 2004; www.fda.gov/cber/minutes/ plague101304t.pdf

170. WebsitePitt ML. Non-human primates as a model for pneumonic plague; Animals Models and Correlates of Protection for Plague Vaccines Workshop; Gaithersburg, MD, USA. 13-14 October 2004; www.fda.gov/cber/minutes/ plague101304t.pdf

171. Elvin, S.J. and E.D. Williamson, Stat 4 but not Stat 6 mediated immune mechanisms are essential in protection against plague. Microb Pathog, 2004. 37(4): p. 177-84.

172. Mittler, R.S., et al., Anti-4-1BB monoclonal antibodies abrogate T cell-dependent humoral immune responses in vivo through the induction of helper T cell anergy. J Exp Med, 1999. 190(10): p. 1535-40. 
173. Foell, J., et al., CD137 costimulatory $T$ cell receptor engagement reverses acute disease in lupus-prone NZB $x$ NZW F1 mice. J Clin Invest, 2003. 111(10): p. 1505-18.

174. Sun, Y., et al., Costimulatory molecule-targeted antibody therapy of a spontaneous autoimmune disease. Nat Med, 2002. 8(12): p. 1405-13.

175. Ganguly, S., et al., Adjuvantive effects of anti-4-1BB agonist Ab and 4-1BBL DNA for a HIV-1 Gag DNA vaccine: different effects on cellular and humoral immunity. Vaccine, 2010. 28(5): p. 1300-9.

176. Wang, C., et al., Immune regulation by 4-1BB and 4-1BBL: complexities and challenges. Immunol Rev, 2009. 229(1): p. 192-215.

177. Amemiya, K., et al., CpG oligodeoxynucleotides augment the murine immune response to the Yersinia pestis F1-V vaccine in bubonic and pneumonic models of plague. Vaccine, 2009. 27(16): p. 2220-9.

178. Slamon, D.J., et al., Studies of the HER-2/neu proto-oncogene in human breast and ovarian cancer. Science, 1989. 244(4905): p. 707-12.

179. Nahta, R. and F.J. Esteva, Herceptin: mechanisms of action and resistance. Cancer Lett, 2006. 232(2): p. 123-38.

180. Nahta, R. and F.J. Esteva, HER2 therapy: molecular mechanisms of trastuzumab resistance. Breast Cancer Res, 2006. 8(6): p. 215.

181. Hall, P.S. and D.A. Cameron, Current perspective - trastuzumab. Eur J Cancer, 2009. 45(1): p. 12-8.

182. Milani, A., et al., Active immunotherapy in HER2 overexpressing breast cancer: current status and future perspectives. Ann Oncol, 2013. 24(7): p. 1740-8.

183. Bernhard, H., et al., Vaccination against the HER-2/neu oncogenic protein. Endocr Relat Cancer, 2002. 9(1): p. 33-44.

184. Disis, M.L., et al., Effect of dose on immune response in patients vaccinated with an her-2/neu intracellular domain protein--based vaccine. J Clin Oncol, 2004. 22(10): p. 1916-25.

185. Limentani S, Dorval T, White $\mathrm{S}$ et al. Phase I dose-escalation trial of a recombinant HER2 vaccine in patients with Stage II/III HER2+ breast cancer. ASCO Meeting Abstracts 2005; 23(16_suppl): 2520.

186. Wang, B., et al., Targeting of the non-mutated tumor antigen HER2/neu to mature dendritic cells induces an integrated immune response that protects against breast cancer in mice. Breast Cancer Res, 2012. 14(2): p. R39.

187. Dubensky, T.W., Jr. and S.G. Reed, Adjuvants for cancer vaccines. Semin Immunol, 2010. 22(3): p. 155-61.

188. Schwarz, T.F., Clinical update of the AS04-adjuvanted human papillomavirus16/18 cervical cancer vaccine, Cervarix. Adv Ther, 2009. 26(11): p. 983-98.

189. Singh, R., et al., Fusion to Listeriolysin $O$ and delivery by Listeria monocytogenes enhances the immunogenicity of HER-2/neu and reveals subdominant epitopes in the FVB/N mouse. J Immunol, 2005. 175(6): p. 3663-73.

190. Seavey, M.M., et al., A novel human Her-2/neu chimeric molecule expressed by Listeria monocytogenes can elicit potent HLA-A2 restricted CD8-positive T cell responses and impact the growth and spread of Her-2/neu-positive breast tumors. Clin Cancer Res, 2009. 15(3): p. 924-32. 
191. Rohrbach, F., et al., Targeted delivery of the ErbB2/HER2 tumor antigen to professional APCs results in effective antitumor immunity. $J$ Immunol, 2005. 174(9): p. 5481-9.

192. Wei, H., et al., Targeted delivery of tumor antigens to activated dendritic cells via CD11c molecules induces potent antitumor immunity in mice. Clin Cancer Res, 2009. 15(14): p. 4612-21.

193. Zizzari, I.G., et al., HER2-based recombinant immunogen to target DCs through FcgammaRs for cancer immunotherapy. J Mol Med (Berl), 2011. 89(12): p. 1231-40.

194. Narayanan, K., et al., Response of established human breast tumors to vaccination with mammaglobin-A cDNA. J Natl Cancer Inst, 2004. 96(18): p. 1388-96.

195. Mandl, S.J., et al., Immunotherapy with MVA-BN(R)-HER2 induces HER-2specific Th1 immunity and alters the intratumoral balance of effector and regulatory $T$ cells. Cancer Immunol Immunother, 2012. 61(1): p. 19-29.

196. Mahmoud, S., et al., CD8(+) T lymphocytes infiltrating breast cancer: $A$ promising new prognostic marker? Oncoimmunology, 2012. 1(3): p. 364-365.

197. Sears, A.K., et al., AE37: a novel T-cell-eliciting vaccine for breast cancer. Expert Opin Biol Ther, 2011. 11(11): p. 1543-50.

198. Sharma, R.K., et al., Tumor cells engineered to codisplay on their surface 4-1BBL and LIGHT costimulatory proteins as a novel vaccine approach for cancer immunotherapy. Cancer Gene Ther, 2010. 17(10): p. 730-41.

199. Cannons, J.L., et al., 4-1BB ligand induces cell division, sustains survival, and enhances effector function of CD4 and CD8 T cells with similar efficacy. $J$ Immunol, 2001. 167(3): p. 1313-24.

200. Watts, T.H., TNF/TNFR family members in costimulation of T cell responses. Annu Rev Immunol, 2005. 23: p. 23-68.

201. Mata-Haro, V., et al., The vaccine adjuvant monophosphoryl lipid A as a TRIFbiased agonist of TLR4. Science, 2007. 316(5831): p. 1628-32.

202. Goldman, B. and L. DeFrancesco, The cancer vaccine roller coaster. Nat Biotechnol, 2009. 27(2): p. 129-39.

203. Cuadros, C., et al., Cooperative effect between immunotherapy and antiangiogenic therapy leads to effective tumor rejection in tolerant Her-2/neu mice. Cancer Res, 2003. 63(18): p. 5895-901.

204. Cuadros, C., et al., Vaccination with dendritic cells pulsed with apoptotic tumors in combination with anti-OX40 and anti-4-1BB monoclonal antibodies induces $T$ cell-mediated protective immunity in Her-2/neu transgenic mice. Int J Cancer, 2005. 116(6): p. 934-43.

205. Lustgarten, J., A.L. Dominguez, and C. Cuadros, The CD8+ T cell repertoire against Her-2/neu antigens in neu transgenic mice is of low avidity with antitumor activity. Eur J Immunol, 2004. 34(3): p. 752-61.

206. Smith, S.E., et al., Signals through 4-1BB inhibit T regulatory cells by blocking IL-9 production enhancing antitumor responses. Cancer Immunol Immunother, 2011. 60(12): p. 1775-87. 
207. Muller, W.J., et al., Single-step induction of mammary adenocarcinoma in transgenic mice bearing the activated c-neu oncogene. Cell, 1988. 54(1): p. 10515.

208. Curiel, T.J., et al., Specific recruitment of regulatory T cells in ovarian carcinoma fosters immune privilege and predicts reduced survival. Nat Med, 2004. 10(9): $\mathrm{p}$. 942-9.

209. Gobert, M., et al., Regulatory T cells recruited through CCL22/CCR4 are selectively activated in lymphoid infiltrates surrounding primary breast tumors and lead to an adverse clinical outcome. Cancer Res, 2009. 69(5): p. 2000-9.

210. Dannull, J., et al., Enhancement of vaccine-mediated antitumor immunity in cancer patients after depletion of regulatory $T$ cells. $J$ Clin Invest, 2005. 115(12): p. 3623-33.

211. Powell, D.J., Jr., et al., Administration of a CD25-directed immunotoxin, LMB-2, to patients with metastatic melanoma induces a selective partial reduction in regulatory T cells in vivo. J Immunol, 2007. 179(7): p. 4919-28.

212. Phan, G.Q., et al., Cancer regression and autoimmunity induced by cytotoxic $T$ lymphocyte-associated antigen 4 blockade in patients with metastatic melanoma. Proc Natl Acad Sci U S A, 2003. 100(14): p. 8372-7.

213. Butler, N.S., et al., Altered IL-4 mRNA stability correlates with Th1 and Th2 bias and susceptibility to hypersensitivity pneumonitis in two inbred strains of mice. $J$ Immunol, 2002. 169(7): p. 3700-9.

214. Smith, I., et al., 2-year follow-up of trastuzumab after adjuvant chemotherapy in HER2-positive breast cancer: a randomised controlled trial. Lancet, 2007. 369(9555): p. 29-36.

215. Sin, J.I., et al., Combined stimulation of TLR9 and 4.1BB augments Trp2 peptide vaccine-mediated melanoma rejection by increasing Ag-specific CTL activity and infiltration into tumor sites. Cancer Lett, 2013. 330(2): p. 190-9.

216. Burgdorf, S., et al., Spatial and mechanistic separation of cross-presentation and endogenous antigen presentation. Nat Immunol, 2008. 9(5): p. 558-66.

217. $\mathrm{Vu}, \mathrm{T}$. and F.X. Claret, Trastuzumab: updated mechanisms of action and resistance in breast cancer. Front Oncol, 2012. 2: p. 62.

218. Yip, Y.L., et al., Identification of epitope regions recognized by tumor inhibitory and stimulatory anti-ErbB-2 monoclonal antibodies: implications for vaccine design. J Immunol, 2001. 166(8): p. 5271-8.

219. Ma, Y., et al., Targeting of antigens to B lymphocytes via CD19 as a means for tumor vaccine development. $J$ Immunol, 2013. 190(11): p. 5588-99.

220. Schulz, E.G., et al., Sequential polarization and imprinting of type $1 \mathrm{~T}$ helper lymphocytes by interferon-gamma and interleukin-12. Immunity, 2009. 30(5): $\mathrm{p}$. 673-83.

221. Lighvani, A.A., et al., T-bet is rapidly induced by interferon-gamma in lymphoid and myeloid cells. Proc Natl Acad Sci U S A, 2001. 98(26): p. 15137-42.

222. Schoenborn, J.R. and C.B. Wilson, Regulation of interferon-gamma during innate and adaptive immune responses. Adv Immunol, 2007. 96: p. 41-101.

223. Ossendorp, F., et al., Importance of CD4(+) Thelper cell responses in tumor immunity. Immunol Lett, 2000. 74(1): p. 75-9. 
224. Schild, H.J., et al., CD4+ helper $T$ cells are required for resistance to a highly metastatic murine tumor. Eur J Immunol, 1987. 17(12): p. 1863-6.

225. Romerdahl, C.A. and M.L. Kripke, Role of helper T-lymphocytes in rejection of UV-induced murine skin cancers. Cancer Res, 1988. 48(9): p. 2325-8.

226. Kast, W.M., et al., Cooperation between cytotoxic and helper Tymphocytes in protection against lethal Sendai virus infection. Protection by $\mathrm{T}$ cells is $\mathrm{MHC}$ restricted and $M H C$-regulated; a model for $M H C$-disease associations. J Exp Med, 1986. 164(3): p. 723-38.

227. Bennett, S.R., et al., Help for cytotoxic-T-cell responses is mediated by CD40 signalling. Nature, 1998. 393(6684): p. 478-80.

228. Ridge, J.P., F. Di Rosa, and P. Matzinger, A conditioned dendritic cell can be a temporal bridge between a CD4+ T-helper and a T-killer cell. Nature, 1998. 393(6684): p. 474-8.

229. Sin, J.I., et al., Intratumoral electroporation of IL-12 cDNA eradicates established melanomas by Trp2(180-188)-specific CD8+CTLs in a perforin/granzymemediated and IFN-gamma-dependent manner: application of Trp2(180-188) peptides. Cancer Immunol Immunother, 2012. 61(10): p. 1671-82.

230. Moore, K.W., et al., Interleukin-10 and the interleukin-10 receptor. Annu Rev Immunol, 2001. 19: p. 683-765.

231. Chen, W.F. and A. Zlotnik, IL-10: a novel cytotoxic T cell differentiation factor. J Immunol, 1991. 147(2): p. 528-34.

232. Cai, G., R.A. Kastelein, and C.A. Hunter, IL-10 enhances NK cell proliferation, cytotoxicity and production of IFN-gamma when combined with IL-18. Eur J Immunol, 1999. 29(9): p. 2658-65.

233. Giovarelli, M., et al., Local release of IL-10 by transfected mouse mammary adenocarcinoma cells does not suppress but enhances antitumor reaction and elicits a strong cytotoxic lymphocyte and antibody-dependent immune memory. $J$ Immunol, 1995. 155(6): p. 3112-23.

234. Zheng, L.M., et al., Interleukin-10 inhibits tumor metastasis through an NK celldependent mechanism. J Exp Med, 1996. 184(2): p. 579-84.

235. Berman, R.M., et al., Systemic administration of cellular IL-10 induces an effective, specific, and long-lived immune response against established tumors in mice. $J$ Immunol, 1996. 157(1): p. 231-8.

236. Fujii, S., et al., Interleukin-10 promotes the maintenance of antitumor CD8(+) Tcell effector function in situ. Blood, 2001. 98(7): p. 2143-51.

237. Sredni, B., et al., Ammonium trichloro(dioxoethylene-o,o')tellurate (AS101) sensitizes tumors to chemotherapy by inhibiting the tumor interleukin 10 autocrine loop. Cancer Res, 2004. 64(5): p. 1843-52.

238. Di Carlo, E., et al., Local release of interleukin-10 by transfected mouse adenocarcinoma cells exhibits pro- and anti-inflammatory activity and results in a delayed tumor rejection. Eur Cytokine Netw, 1998. 9(1): p. 61-8.

239. Adams, S., Toll-like receptor agonists in cancer therapy. Immunotherapy, 2009. 1(6): p. 949-64.

240. Woo, E.Y., et al., Regulatory CD4(+)CD25(+) T cells in tumors from patients with early-stage non-small cell lung cancer and late-stage ovarian cancer. Cancer Res, 2001. 61(12): p. 4766-72. 
241. Barnett, B.G., et al., Regulatory T cells: a new frontier in cancer immunotherapy. Adv Exp Med Biol, 2008. 622: p. 255-60.

242. Liu, F., et al., Peritumoral FOXP3(+) regulatory T cell is sensitive to chemotherapy while intratumoral FOXP3(+) regulatory $T$ cell is prognostic predictor of breast cancer patients. Breast Cancer Res Treat, 2012. 135(2): p. 459-67.

243. Demir, L., et al., Predictive and prognostic factors in locally advanced breast cancer: effect of intratumoral FOXP3+ Tregs. Clin Exp Metastasis, 2013. 30(8): p. 1047-62.

244. Elpek, K.G., et al., CD4+CD25+ T regulatory cells dominate multiple immune evasion mechanisms in early but not late phases of tumor development in a B cell lymphoma model. J Immunol, 2007. 178(11): p. 6840-8.

245. Klages, K., et al., Selective depletion of Foxp3+ regulatory T cells improves effective therapeutic vaccination against established melanoma. Cancer Res, 2010. 70(20): p. 7788-99.

246. Zhou, Q., et al., Depletion of endogenous tumor-associated regulatory T cells improves the efficacy of adoptive cytotoxic T-cell immunotherapy in murine acute myeloid leukemia. Blood, 2009. 114(18): p. 3793-802.

247. Liu, Y., et al., Adenovirus-mediated intratumoral expression of immunostimulatory proteins in combination with systemic Treg inactivation induces tumor-destructive immune responses in mouse models. Cancer Gene Ther, 2011. 18(6): p. 407-18.

248. Zheng, G., B. Wang, and A. Chen, The 4-1BB costimulation augments the proliferation of CD4+CD25+ regulatory $T$ cells. $J$ Immunol, 2004. 173(4): p. 2428-34.

249. Gonzalez-Navajas, J.M., et al., TLR4 signaling in effector CD4+ $T$ cells regulates TCR activation and experimental colitis in mice. $J$ Clin Invest, 2010. 120(2): p. 570-81.

250. Caramalho, I., et al., Regulatory $T$ cells selectively express toll-like receptors and are activated by lipopolysaccharide. J Exp Med, 2003. 197(4): p. 403-11.

251. Uno, T., et al., Eradication of established tumors in mice by a combination antibody-based therapy. Nat Med, 2006. 12(6): p. 693-8.

252. Halstead, E.S., et al., In vivo stimulation of CD137 broadens primary antiviral CD8+ T cell responses. Nat Immunol, 2002. 3(6): p. 536-41.

253. Melero, I., et al., Monoclonal antibodies against the 4-1BB T-cell activation molecule eradicate established tumors. Nat Med, 1997. 3(6): p. 682-5.

254. Niu, L., et al., Cytokine-mediated disruption of lymphocyte trafficking, hemopoiesis, and induction of lymphopenia, anemia, and thrombocytopenia in anti-CD137-treated mice. J Immunol, 2007. 178(7): p. 4194-213.

255. Romanowski, B., et al., Sustained efficacy and immunogenicity of the human papillomavirus (HPV)-16/18 AS04-adjuvanted vaccine: analysis of a randomised placebo-controlled trial up to 6.4 years. Lancet, 2009. 374(9706): p. 1975-85. 


\section{CURRICULUM VITA}

NAME: $\quad$ Gunes Dinc

ADDRESS: Department of Microbiology \& Immunology

570 S. Preston Street, Build. Baxter I, 4th Floor

University of Louisville

Louisville, KY 40206

DOB: $\quad$ Ankara, Turkey- May 23, 1984

EDUCATION

\& TRAINING:

B.S., Biology
Uludag University

2003-2007

M.S., Microbiology \& Immunology

University of Louisville

2009-2011

Ph.D., Microbiology \& Immunology

University of Louisville

2009-2014

AWARDS: Undergraduate Top Ranking Student Award (Honor student)

Uludag University, Bursa, Turkey

2007

Graduate Scholarship for Study Abroad

Turkish Ministry of National Education

2008-2013

Condict Moore Graduate Student Research First Place Award 11th Annual Brown Cancer Center Retreat, James Graham Brown Cancer Center, University of Louisville, Louisville 2012

PROFESSIONAL SOCIETIES: American Association of Immunologists 


\section{PUBLICATIONS:}

1. Srivastava AK, Sharma RK, Yolcu ES, Ulker V, MacLeod K, Dinc G, Shirwan H. Prime-boost vaccination with SA-4-1BBL costimulatory molecule and survivin eradicates lung carcinoma in CD8+ $\mathrm{T}$ and $\mathrm{NK}$ cell dependent manner. PLoS One, 7(11), 2012. PMID: 2314488

2. Dinc $\mathrm{G}$, et al. Improving the cellular efficacy of the lead Yersinia pestis $\mathrm{rF1V}$ subunit vaccine using SA-4-1BBL as a novel adjuvant. Under review in Vaccine.

3. Dinc G, et al. Vaccination with rat Her-2/neu and combined adjuvant system of SA-4$1 \mathrm{BBL}$ and MPL eradicates established breast tumors in BALB/c and tolerogenic BALB/neuT mice. Manuscript ready for submission.

4. Srivastava AK, Dinc G, et al. SA-4-1BBL and MPL combination as an adjuvant system shows robust therapeutic efficacy by increasing intratumoral $\mathrm{CD}^{+} \mathrm{T}$ effector/CD4 ${ }^{+}$Foxp $^{+}$T regulatory cell ratio. Manuscript ready for submission.

\section{NATIONAL \& LOCAL MEETINGS PRESENTATIONS}

1. $11^{\text {th }}$ Annual Brown Cancer Center Retreat, 2012, Olmsted, Louisville, KY

Dinc G, et al. SA-4-1BBL and monophosphoryl lipid A as a novel adjuvant system with potent therapeutic efficacy against cancer in the absence of detectable toxicity and autoimmunity.

2. American Association of Immunologists (AAI) Annual Meeting, 2013, Honolulu, HI. Dinc G, et al. SA-4-1BBL and monophosphoryl lipid A as a novel adjuvant system with potent therapeutic efficacy against cancer in the absence of detectable toxicity and autoimmunity. 